San Jose State University

SJSU ScholarWorks

Master's Theses

Master's Theses and Graduate Research

1992

\title{
Fabrication and performance of AC thin-film electroluminescent devices using MOCVD prepared Zns:Mn phosphor layers
}

\author{
Da-Zen Chuang \\ San Jose State University
}

Follow this and additional works at: https://scholarworks.sjsu.edu/etd_theses

\section{Recommended Citation}

Chuang, Da-Zen, "Fabrication and performance of AC thin-film electroluminescent devices using MOCVD prepared Zns:Mn phosphor layers" (1992). Master's Theses. 298.

DOI: https://doi.org/10.31979/etd.5qav-jcsh

https://scholarworks.sjsu.edu/etd_theses/298

This Thesis is brought to you for free and open access by the Master's Theses and Graduate Research at SJSU ScholarWorks. It has been accepted for inclusion in Master's Theses by an authorized administrator of SJSU ScholarWorks. For more information, please contact scholarworks@sjsu.edu. 


\section{INFORMATION TO USERS}

This manuscript has been reproduced from the microfilm master. UMI films the text directly from the original or copy submitted. Thus, some thesis and dissertation copies are in typewriter face, while others may be from any type of computer printer.

The quality of this reproduction is dependent upon the quality of the copy submitted. Broken or indistinct print, colored or poor quality illustrations and photographs, print bleedthrough, substandard margins, and improper alignment can adversely affect reproduction.

In the unlikely event that the author did not send UMI a complete manuscript and there are missing pages, these will be noted. Also, if unauthorized copyright material had to be removed, a note will indicate the deletion.

Oversize materials (e.g., maps, drawings, charts) are reproduced by sectioning the original, beginning at the upper left-hand corner and continuing from left to right in equal sections with small overlaps. Each original is also photographed in one exposure and is included in reduced form at the back of the book.

Photographs included in the original manuscript have been reproduced xerographically in this copy. Higher quality $6 "$ " 9 9" black and white photographic prints are available for any photographs or illustrations appearing in this copy for an additional charge. Contact UMI directly to order.

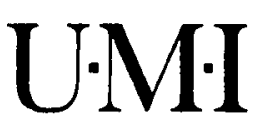

University Microfilms International

A Bell \& Howell Information Company

300 North Zeeb Road. Ann Arbor, Mi 48106-1346 USA

313/761-4700 800/521-0600 

Order Number 1848659

Fabrication and performance of $\mathrm{AC}$ thin-film electroluminescent devices using MOCVD prepared ZnS:Mn phosphor layers

Chuang, Da-Zen, M.S.

San Jose State University, 1992

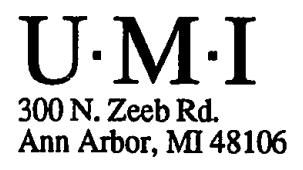




\title{
FABRICATION AND PERFORMANCE OF AC THIN-FILM ELECTROLUMINESCENT DEVICES USING MOCVD PREPARED ZnS:Mn PHOSPHOR LAYERS
}

\author{
A Thesis \\ Presented to \\ The Faculty of the Department of Materials Engineering \\ San Jose State University \\ In Partial Fulfillment \\ of the Requirements for the Degree \\ Master of Science \\ By
}

Da-Zen Chuang

May, 1992 
Approved for the Department of Materials Engineering
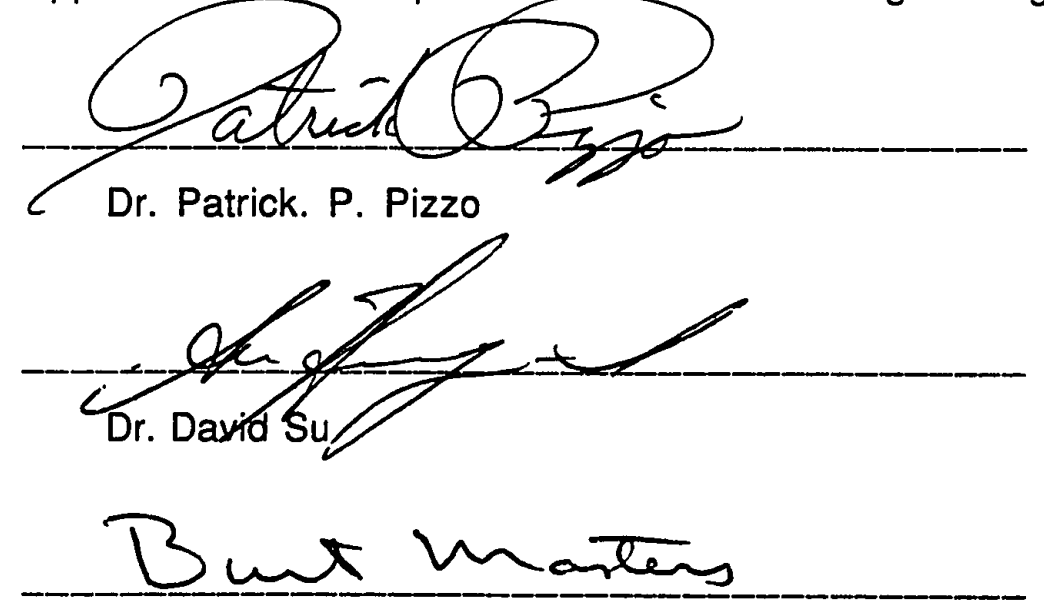

Dr. Burt Masters

Approved for the University

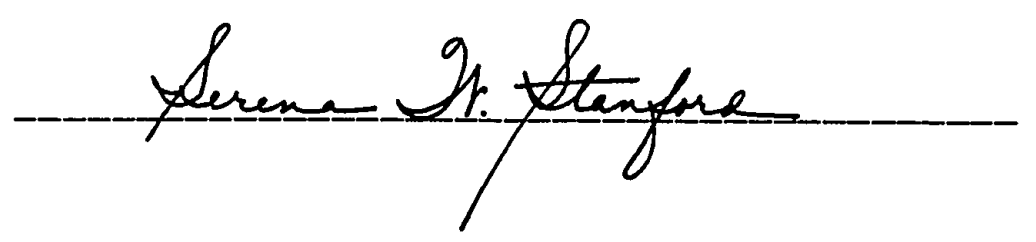




\begin{abstract}
Thin film electroluminescent devices (TFEL) with the metalinsulator-semiconductor-insulator-metal structure (MISIM) were made and studied in this work. MOCVD prepared $\mathrm{ZnS}$ was used as a phosphor layer after the introduction of manganese centers by thermal diffusion; vacuum evaporated SiO was chosen for the insulating layers; the whole device was fabricated on an ITO glass substrate. A sinusoidal power supply was connected to the devices to apply voltage for electroluminescence (EL) generation. $E L$ was created as the applied voltage exceeded threshold value, which was 100 to $220 \mathrm{rms}$ volts. The breakdown field of the devices was typically in the range of 170 to $260 \mathrm{rms}$ volts. The emission waveform of EL was observed to be unsymmetrical with respect to the polarity of the applied voltage. In addition, the luminance v.s. applied voltage curves exhibited a hysteresis or memory effect, similar to that reported by other researchers. The average lifetime of the prepared devices was about two to three hours. This short life was thought to be due to the degradation of the SiO layers and metal films. Reproducibility of the prepared devices was poor. Replacing the SiO with an insulator having a high melting point and good charge capacitance, together with better control of the device fabrication might be of help to prolong the lifetime and increase the reproducibility of ac TFEL devices using the proposed fabrication process.
\end{abstract}




\section{Table of Contents}

Page

Abstract III

Table of Contents IV

List of Figures $\quad$ VI

List of Tables VIII

Acknowledgements IX

1. Introduction 1

2. Theories of Luminescence Generation of TFEL Devices 5

2.1. Device Structure 5

2.2. Mechanisms of Electroluminescence 7

2.3. Impact Excitation of Luminescent Centers 10

2.4. Memory Effect and Charge Transfer 18

$\begin{array}{ll}\text { 2.5. Summary } & 24\end{array}$

3. Choice of Materials and Fabrication Technologies 26

$\begin{array}{ll}\text { 3.1. Phosphor Layer } & 26\end{array}$

3.2. Dopants 32

3.3. Color EL Displays $\quad 34$

3.4. Deposition of ZnS:Mn 36

3.5. MOCVD ZnS Layer 43

3.6. Insulating Layers 46

3.7. Transparent Conductor and Glass Substrate 52

IV. 
3.8 Summary 53

4. Experimental Procedures 56

4.1. Device Fabrication 56

4.2. Characterization Methods 62

5. Experimental Results and Discussion 65

6. Conclusion $\quad 77$

$\begin{array}{ll}\text { 7. References } & 79\end{array}$

$\begin{array}{ll}\text { Appendixes } & 94\end{array}$

A. Thickness Distribution of Prepared ZnS Film Using MOVCD 94

B. The Estimation of Evaporated Mn content in ZnS:Mn 97

C. The Analysis of Mn Content in ZnS:Mn 101

D. The Oxygen Content in ZnS:Mn 103 


\section{List of Figures}

Page

1. The schematic diagram of ac TFEL devices with MISIM

6 structure.

2. The energy band diagram of ac TFEL devices with MISIM structure. The mechanisms of EL generation can be illustrated in sequence as: (a) tunneling of electrons from the interface states into the conduction band of phosphor, (b) impact excitation of luminescent centers by hot electrons, (c) impact ionization of host lattices, and (d) the trapping of electrons in the opposite interface.

3. A schematic diagram showing the tunneling of electrons from the interface states into the conduction band of phosphor.

4. (a) The brightness efficiency-Mn concentration characteristic and (b) the relationship of the decay time v.s. $\mathrm{Mn}$ concentration of $\mathrm{ZnS}: \mathrm{Mn}$.

5. Schematic illustration of (a) the generation of EL, which is formed as excited luminescent centers return to ground state radiatively; (b) Auger effect, where the outermost electron is ejected by the absorption of energy transferred from excited luminescent centers via non-radiative paths.

6. (a) A side view of an ac TFEL device using double-insulating- 21 layer structure, and (b) the hysteresis effect of the ac TFEL devices.

7. A schematic illustration of (a) the top view and side view of patterned phosphor structure, and (b) patterned filter method. 
8. The chemical structure of (a) TCM, (b) CPM, and (c) BCPM. 45

9. Process sequence of fabricating ac TFEL devices.

10. Schematic illustration of MOCVD reactor used in this work. 59

11. The schematic diagram of experimental set-up for

63 measuring and recording relative brightness, voltage and current.

12. The typical luminance-voltage characteristic of MOCVD prepared $\mathrm{ZnS}: \mathrm{Mn}$ ac TFEL devices under $1 \mathrm{k} \mathrm{Hz}$ sinusoidal excitation.

13. The current-voltage characteristic of ac TFEL devices 66 under $1 \mathrm{k} \mathrm{Hz}$ sinusoidal power.

14. The emission waveform of ac TFEL devices(lower) under $1 \mathrm{k} \mathrm{Hz}$ sinusoidal voltage excitation(upper).

15. The relative intensity of generated EL of the leading edge and the trailing edge with respect to the applied voltage.

16. The typical hysteretic behavior of luminance-voltage characteristic of ac TFEL devices.

17. The change of luminance-voltage characteristic of ac TFEL 74 devices with respect to the time of power applied.

18. The relative position of deposition center of MOCVD ZnS. 94

19. A schematic illustration of the position of deposition center on ITO glass and the relative positions where measurements of device thickness are made.

20. The thickness distribution of MOCVD ZnS.

21. A schematic illustration of small-area source approach. 


\section{List of Tables}

page

1. Color and brightness of ac TFEL devices using doped ZnS, $\mathrm{SrS}$ and $\mathrm{CaS}$ as phosphor.

2. The energy band gap of some host materials usually used. $\quad 30$

3. Physical properties of some thin dielectric films usually 49 used in ac TFEL devices.

4. Testing results on prepared ac TFEL devices using 67 sinusoidal power with $\mathrm{f}=1 \mathrm{k} \mathrm{Hz}$.

5. The thickness distribution of MOCVD ZnS. 96

6. Analysis of $\mathrm{Mn}$ content in $\mathrm{ZnS}: \mathrm{Mn}$ using Electron Microprobe. 101

7. Analysis of oxygen content in $\mathrm{ZnS}: \mathrm{Mn}$. 


\section{Acknowledgements}

I wish to express my sincere gratitude to Professor Burt Masters, my thesis advisor, for his guidance and assistance throughout this investigation, and his willingness to listen to a graduate student's thoughts. I am also grateful to Professor Patrick P. Pizzo and Dr. David Su for their advice and serving on my reading committee.

My deepest appreciation goes to my parents. Thanks for their endless tolerance, encouragement and support of my study abroad. The support from my brother and sister is warmly acknowledged. Many thanks to my wife (to be) for her assistance and typing part of the original draft.

I wish to thank Jenny Hyuang and Linda Hyuang for the loan of their Macintosh and assistance during thesis writing. I also express my appreciation to my colleague, Waley Sung, for his help of the use of equipment in the IC lab, and to Dennis Ramos, for his analysis of the impurity content in the devices. 
In memory of my grandmother

$$
X \text {. }
$$




\section{Introduction}

With excellent viewing angles, multi-color capability, good contrast, and low cost, the cathode-ray tube (CRT) has been the dominant technology of display for 85 years. However, the development of a higher resolution CRT to meet the requirements of the next generation TV, high definition TV (HDTV), ran into two major problems: the lack of brightness and the generation of unwanted heat. In addition, weight and size of traditional CRTs limit their applications in today's market where light weight and thin thickness of displays are becoming more desirable. Flat panel display (FPD) is an ideal alternative if all of these requirements on display are matched. An FPD can be defined as an electric display with a thickness which is a small fraction of its viewing area. There are seven major techniques for making FPD: gas-plasma display, liquid crystal display (LCD), electroluminescent (EL) display, lightemitting diode (LED) display, vacuum fluorescent display, flat CRT display and electrochromic display. Of all these FPD technologies, only the first three are suitable for manufacturing dot-matrix displays that can reproduce sizable amounts of information with high resolution.

Like fluorescent lamps, gas-plasma displays are operated by exciting a gas, usually neon or argon-neon mixture, through the application of voltage. There are typically three different plasma 
technologies: dc refresh, ac refresh, and ac memory. The dc type is easier to produce but usually has an inherent background glow due to the continuously present dc voltage necessary for refresh. The acmemory display contains dielectric layers that separate the gas from the activating electrodes. This makes ac-type-plasma displays more complicated to fabricate but background glow is eliminated and no image-refresh is required. The possibility of very large sizes, wide viewing angle and memory functions are the advantages of gasplasma display. Full color plasma displays are under development. The major problem with color plasma is its short lifetime due to the contamination of surface by sputtered materials. Moreover, higher power consumption caused by low luminous efficiency, and aesthetically unpleasing colors are problems restricting the development of this technology.

LCD is the most widely used FPD and is now the leading technology of all the FPD techniques. The operation of an LCD is mainly based on the electronically induced reorientation of crystal structure in mobile liquid crystal(1). In addition to the oldest twisted-nematic-type (TN) liquid crystal, the supertwisted-nematic (STN), double-supertwisted-nematic (DSTN) and optical-modeinterference (OMI) liquid crystals are the other techniques. Generally speaking, each LCD technique enjoys the advantages of low power (if backlighting isn't used) and ambient light readability. However, narrow viewing angle and limited matrix addressability have been the problems of LCD for a long time. Recently, the development of 
active matrix thin film transistor (TFT) LCD has emerged as the most important technique to solve those problems. Multi-color is possible and is developed by the incorporation of organic filters. The major difficulty in making active matrix TFT LCD lies in the requirement of depositing up to three million transistors on a large glass plate with nearly zero defects. Low yield, said to be around 20 percent $^{(2)}$, associated high cost, and limited size of display become the chief problems of active matrix TFT LCD.

Among these three major FPD technologies, electroluminescence is the only fully solid-state technique, requiring no gas or backlite. Although electroluminescence has been knows for more than 50 years, it was not until 1974 that the first long lifetime and good brightness EL display was successfully made ${ }^{(3)}$. There are now two principle types of EL display available: the alternating current thinfilm electroluminescent (ac TFEL) display and the direct current thick-film electroluminescent (DCEL) display. DCEL displays are essentially resistive devices; ac TFEL displays, which contain dielectric layers, are capacitive devices and are structurally more complicated than the former. The major advantage of DCEL is its lower manufacturing cost due to, of course, its simple structure. However, its long term stability is still a problem which seems hard to overcome. Ac TFEL displays offering high speed, good readability, excellent contrast, and wide viewing angles are now running only behind LCDs in FPDs' market, and appear to have a good chance of 
overcoming TFT LCDs in those applications where large display size is required.

The objective of this work is to carry out the design, fabrication, and testing of ac TFEL devices using the facilities available in San Jose State University's "Thin Film Laboratory." In the following section, the theories of EL generation from ac TFEL devices with double-insulating-layer structure will be discussed. In section 3, the choice of materials and the fabrication techniques usually used for each layer in ac TFEL devices will be described. The procedures of fabricating EL devices using MOCVD ZnS will be presented in section 4; the experimental results in this work will be listed and discussed in section 5. The whole work is concluded in section 6. Detailed experimental information dealing with this thesis is included in the Appendixes. 


\section{Theories of Light Generation of TFEL Devices}

\subsection{Device Structure}

Intrinsic electroluminescence (EL), discovered by Destrian in $1936(4)$, is the phenomenon that visible light can be generated by exposing phosphor materials to high electric fields. Because thin phosphor films are usually exposed to high voltage, EL devices are associated with possible degradation or dielectric breakdown. If there is an insulating layer next to the phosphor layer, the amount of charge per unit area passing through the phosphor film can be limited to a certain range and the breakdown of the phosphor film can then be suppressed. It is for this reason that, after being first used by M. J. Russ and D. I. Kennedy(5), the so called doubleinsulating- layer structure is widely accepted for ac TFEL devices.

The basic structure of ac TFEL devices using the doubleinsulating-layer structure is shown in Fig.1. It consists of a phosphor layer sandwiched between two insulating layers, an aluminum film as top electrode, and a transparent conductive film as back electrode. The whole device is built on a glass substrate. An ac voltage supply is connected to the electrodes. When the applied field exceeds the threshold value, say $10^{6} \mathrm{~V} / \mathrm{cm}$ for $\mathrm{ZnS}: \mathrm{Mn}, \mathrm{EL}$ can be generated from the phosphor film. This structure is also called the metal-insulator-semiconductor-insulator-metal (MISIM) structure. 


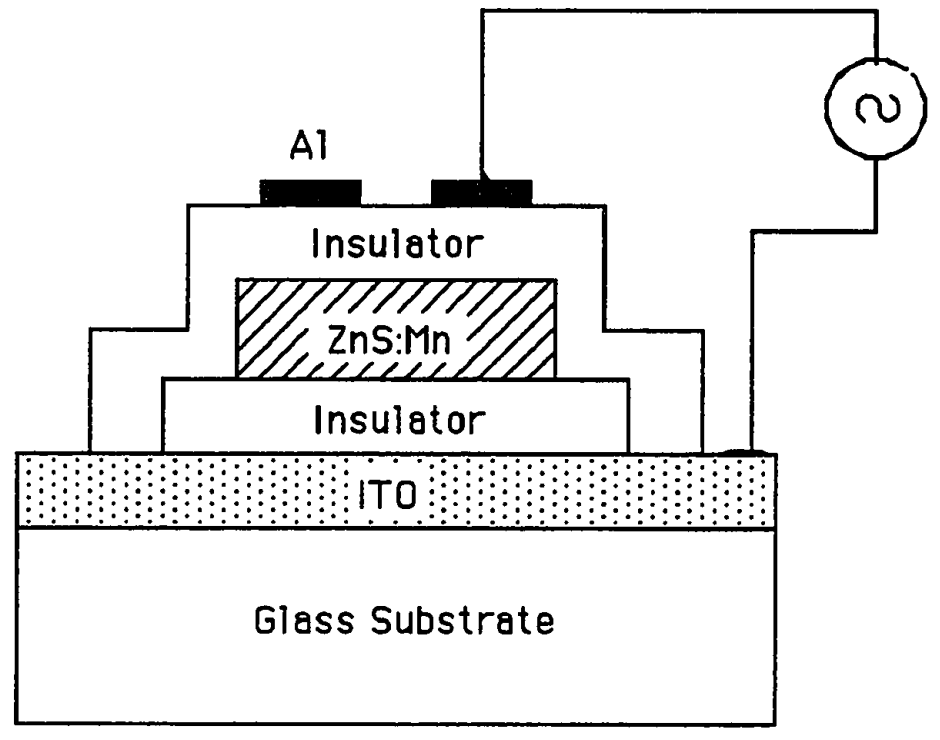

Fig. 1 The schematic diagram of ac TFEL devices with the MISIM structure.

The need of high driving voltage is one of the serious problems with ac TFEL devices. The use of a metal-insulator-semiconductormetal (MISM) structure was suggested as one way to reduce the driving voltage of ac TFEL devices(6)(7). However, the associated low efficiency has blocked further research of TFEL devices using the MISM structure(8). 
In this thesis work, ac TFEL devices are made with the MISIM structure. Any theories and models about ac TFEL devices mentioned in this thesis are mainly based on the MISIM structure.

\subsection{Mechanisms of Electroluminescence}

Basically, each luminescence process, like photoluminescence and cathodoluminescence, involves (1) absorption of energy; (2) excitation of local centers; and finally (3) emission of energy in forms of radiation with wavelength in the visible portion of the spectrum. People have known a great deal about the mechanisms involved in the first step, but less about excitation. The model, direct impact excitation of luminescent centers by hot electrons, after first being proposed by $\mathrm{Krupka}^{(9)}$, and later modified by

numerous researchers $(10)(11)(12)(13)$, is believed to be the way EL generates.

The energy band diagram of a TFEL device with the MISIM structure is shown in Fig. 2. Although the initial source of electrons in this device is still not well understood, electrons are believed to exist in the interface of insulator and phosphor layer due to interruption of the periodic lattice structure, lattice mismatch, non-stoichiometry, and dangling chemical bonds(14). If a bias field is applied on the electrodes of a TFEL device, say, momentary cathode in the left, electrons can be tunnel-emitted from deep traps in interface states near the momentary cathode into the conduction 


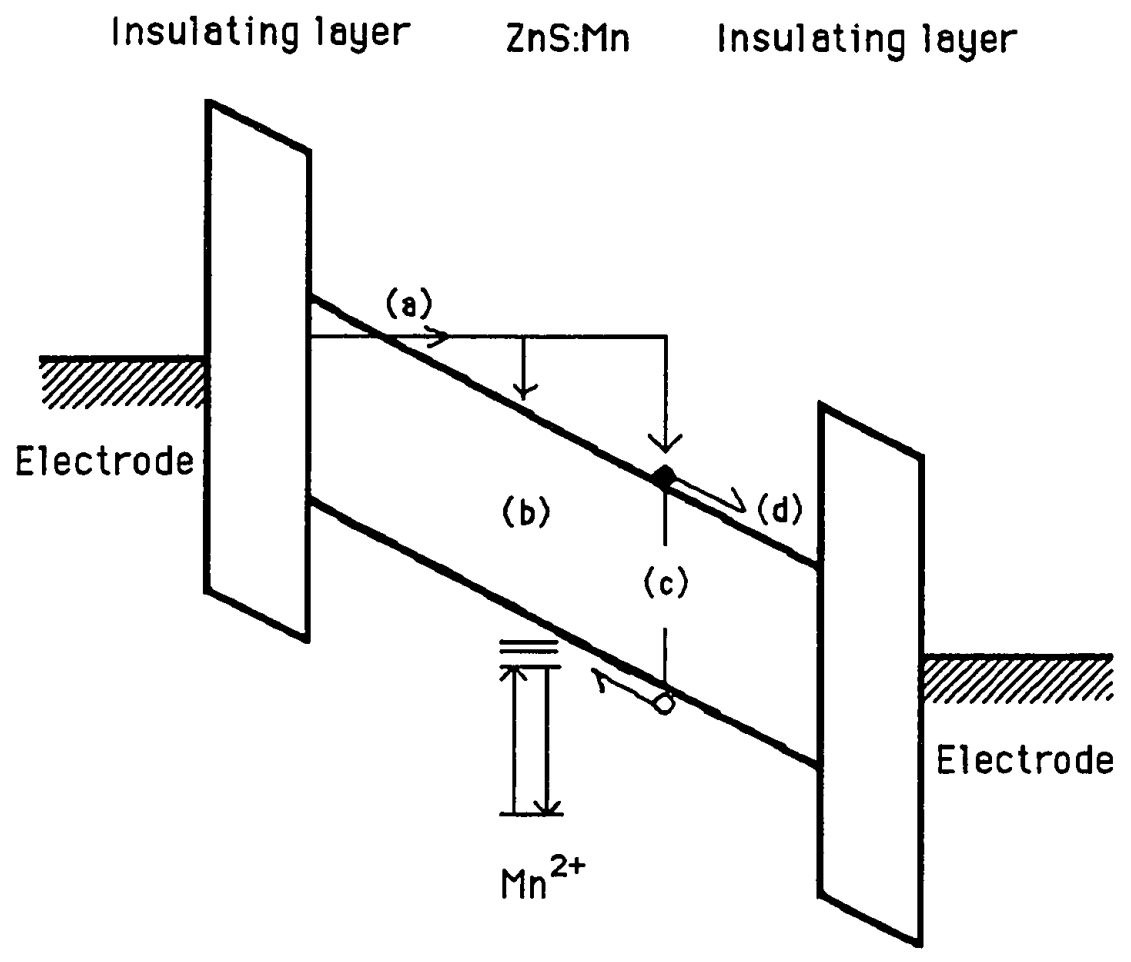

Fig. 2 The energy band diagram of an ac TFEL device with MISIM structure. The mechanisms of EL generation can be illustrated in sequence as: (a) tunneling of electrons from the interface states into the conduction band of phosphor, (b) impact excitation of luminescent centers by hot electrons, (c) impact ionization of host lattices, and (d) the trapping of electrons in the opposite interface. 
band of the phosphor, and are subsequently accelerated by the applied electric field. These electrons are usually called hot electrons and are shown in Fig. 2(a). Some of these hot electrons with sufficient energies can impact-excite the electrons of luminescent centers which soon return to the ground state and release gained energies in forms of radiation, and some of the more energetic electrons can impact-excite electrons in the valence band of the phosphor, producing electron-hole pairs. This generated light is called electroluminescence(EL). Mechanisms (b) and (c) in Fig. 2 show the impact excitation of luminescent centers and impact ionization of the lattice by hot electrons, respectively. Electrons after being multiplied by impact ionization will arrive at the opposite side, the momentary anode, and are trapped again in the interface between the insulator and the phosphor layer as shown in Fig. 2(d). When the polarity of this interface switches inversely from anode into cathode, these electrons are again tunnel-emitted and accelerated into the conduction band of phosphor and the whole process restarts successively until the applied field is released.

The above picture of EL generation is mainly based on $\mathrm{Mn}$ doped $\mathrm{ZnS}(\mathrm{ZnS}: \mathrm{Mn})$. Deviation from the model for $\mathrm{ZnS}: \mathrm{Mn}$ has been proposed in the case of $\mathrm{ZnS}: \mathrm{Tb}^{3+}$, where energy transfer from the $\mathrm{ZnS}$ host would also take a part in the excitation of $\mathrm{Tb}$ centers, together with impact excitation(12). Other mechanisms have also been found on ac TFEL devices using SrS: $\mathrm{Ce}^{3+}$ and $\mathrm{CaS}: \mathrm{Eu}^{2+}$ as the phosphor layer(15). In those cases, the efficient capture of conduction electrons by 
ionized luminescent centers is believed to be the way luminescent centers get energy. However, there are still many steps of EL generation which are common among $\mathrm{ZnS}$ and other phosphors. The theories of EL generation discussed on the following sections are based mainly on our understanding of $\mathrm{ZnS}: \mathrm{Mn}$ using the MISIM structure.

\subsection{Impact Excitation of Luminescent Centers}

For the model of direct impact excitation, electrons, which are trapped in energy levels in the band gap and located at the cathodic phosphor-insulator interface, can tunnel through the barrier into the phosphor. Tunneling results from the exponential fall off of the electron wave function in the forbidden gap of phosphor(11). Because the luminance and electrical characteristic of ac TFEL devices are quite insensitive to temperature, the possibility of lowering of the potential well by a thermally induced process such as the FrenklePoole effect can be ruled out(10)(16). Tunneling from a deep level depends strongly on the detailed shape of the trapping potential and is dominated by the Wentzel-Kramers-Brillouin (WKB) transparency-the tunneling probability of electrons--of the barrier. For tunneling of electrons from a Dirac well into the conduction band of phosphors, we can express the emission rate of electrons, $e_{n}$, as 


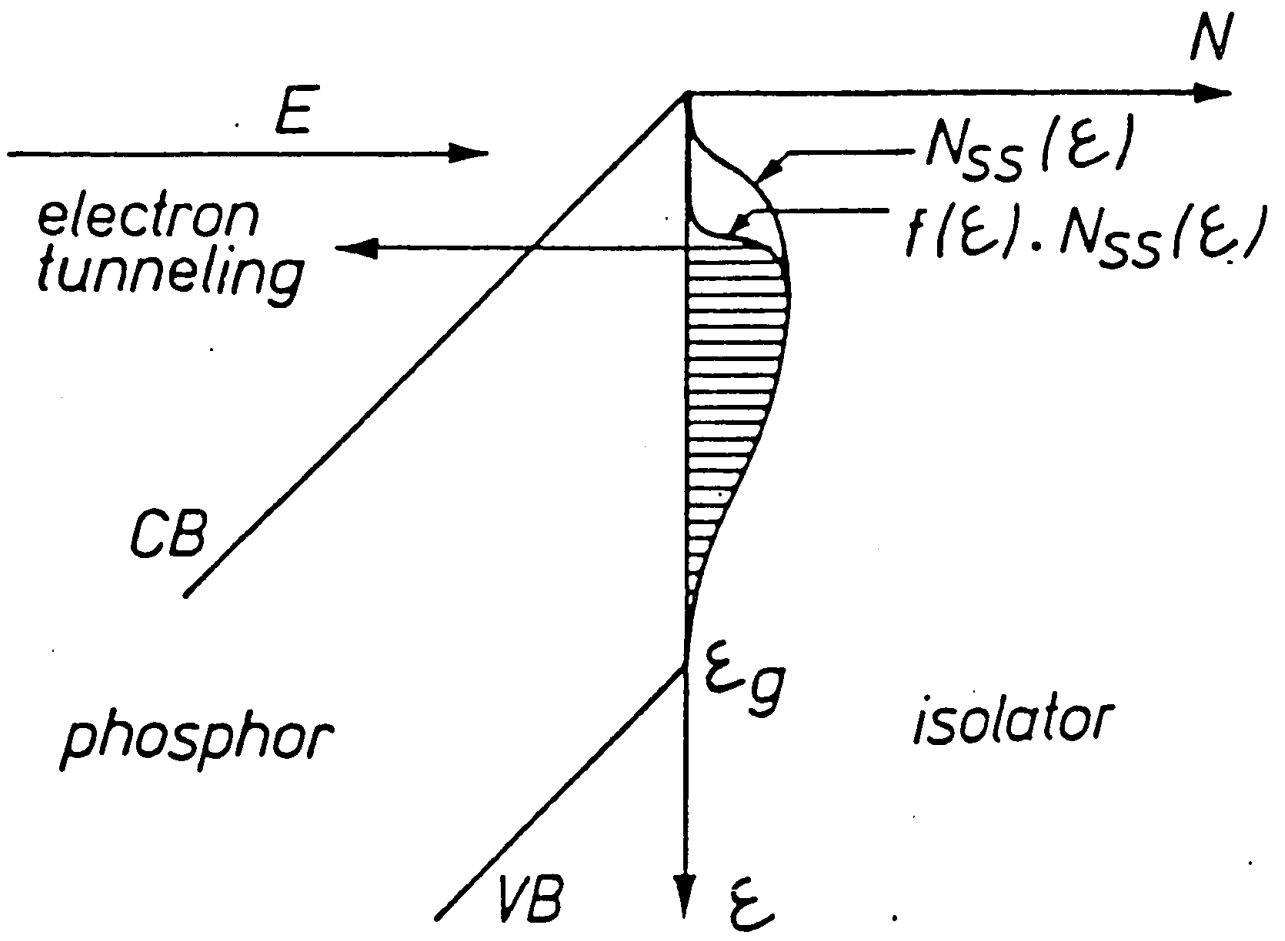

Fig. 3 The schematic diagram showing the tunneling of electrons from the interface states into the conduction band of phosphor(19).

$$
\begin{aligned}
e_{n} & =K \times \operatorname{Exp}\left(-4\left(2 m^{*}\right)^{1 / 2} \times A^{3 / 2} / 3 q h F\right) \\
K & =q F / 4\left(2 m^{\star} A\right)^{1 / 2}
\end{aligned}
$$

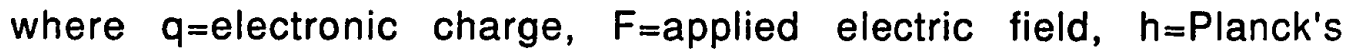
constant, $m^{*}=$ electron effective mass and $A=$ depth of Dirac well(17). 
The exponential term of Eq. 1 is the WKB transparency of the barrier and $K$ is a multiple constant ${ }^{(18)}$. Therefore, the current $J$ of electrons tunneling from the interface under electric field $E$ is

$$
J=\int_{0}^{E g}\left(q \times f(E) \times N(E) \times e_{n}\right) d E
$$

where Eg is the band gap energy, and $f(E)$ and $N(E)$ are the distribution function and density of states at energy level $E$, respectively(19). The term $f(E) \times N(E) \times d E$ in Eq. 1 expresses the number of available electrons as shown in Fig. 3.

When the luminescent centers in the phosphor are impactexcited by tunneling hot electrons, the intensities of radiative emission which follow are dependent on a convolution of the impact cross section $S$ and the hot-electron distribution function $f$. The rate of excitation $R_{e}$ of luminescent centers by the energetic electrons can be written in a simple approximation

$$
R_{e}=n \times N_{1} \int(f(E) \times V(E) \times S(E)) d E
$$

where $N_{1}$ is the concentration of centers and $S(E)$ is the impact cross section of luminescent centers at associated electron energy $E$. $n$ and $V(E)$ are the density and velocity of hot electrons, respectively(20). 
Hot-electron energy distribution is a function of the concentration of luminescent centers and was proposed to fit a Maxwell-Boltzmann-Druyvesteyn (MBD) distribution, which indicated that the probability of finding an electron with energy $E$ had an exponential dependence on $E$ and could be expressed by $f(E)=C \times$ Exp $\left(-E^{2} / \Delta^{2}\right)$, where $C$ was a constant and $\Delta$ was the characteristic energy(21). Both the simplified and more accurate calculations of impact cross section of luminescent centers, based on $\mathrm{Mn}^{2+}$ and $\mathrm{Er}^{3+}$ in $\mathrm{ZnS}$, have been worked out to be on the order of $10^{-16} \mathrm{~cm}^{2}$, which is in agreement with the experimental result, $4 \times 10^{-16} \mathrm{~cm}^{2(11)(22)(23)}$. From MBD's theories, impact cross section is determined by the electric dipole transition and is proportional to the radiative transition probability.

$E L$ is generated after those excited luminescent centers return to their ground states. Most efficient EL involves transition in the inner shells of the luminescent centers, such as the $3 d$ shell of $\mathrm{Mn}^{2+}$ and the $4 f$ shell of rare earth $\mathrm{Tb}^{3+}$ and $\mathrm{Er}^{3+}{ }^{34)} . \mathrm{Mn}^{2+}$ doped $\mathrm{ZnS}$ ( $\mathrm{ZnS}: \mathrm{Mn})$, the most often used phosphor, has a ${ }^{6} \mathrm{~A}_{1}$ ground state which is located below the valence band of $\mathrm{ZnS}$, and has totally 5 possible excitation paths. Among those paths, the transition of ${ }^{4} T_{1}\left({ }^{4} G\right)$, the first excited state, to the ground state of $\mathrm{Mn}^{2+}$ is responsible for the emission of yellow EL of $\mathrm{ZnS}: \mathrm{Mn}$ (see also section 3). The lifetime of $\mathrm{Mn}^{*}$, the excited $\mathrm{Mn}^{2+}$, is $1 \mathrm{msec}$ for low $\mathrm{Mn}^{2+}$ concentration. This long life is due to the fact that the transitions are all spin and parity forbidden in the isolated $\mathrm{Mn}^{2+}$ centers so that the radiative 
decay is rather slow(10)(11). At low electric-excitation level (threshold EL), the decay of brightness $B$ was found to be fit to an empirical two-parameter law

$$
B(t)=B_{0} \times \operatorname{Exp}(-t / T)^{a}
$$

where $T$, the radiative lifetime of $\mathrm{Mn}^{2+}$, and a, an empirical constant, are strongly dependent on the $\mathrm{Mn}$ concentration(25).

Generally speaking, for $\mathrm{ZnS}: \mathrm{Mn}$, isolated $\mathrm{Mn}$ centers are more efficient for EL generation than clustered centers, which might contain 2 to $4 \mathrm{Mn}$ atoms ${ }^{(26)}$. Radiative emission of $\mathrm{ZnS}: \mathrm{Mn}$ at high dopant concentration will be reduced by the enhancement of energy transfer processes like concentration quenching and Auger quenching discussed below.

Fig. $4(a)$ shows the typical characteristic of brightness efficiency v.s. $\mathrm{Mn}^{2+}$ concentration of $\mathrm{ZnS}: \mathrm{Mn}^{(13)}$. We can easily find that the efficiency of brightness of EL does not increase exactly with increasing $\mathrm{Mn}$ concentration. Maximum efficiency is reached at a $\mathrm{Mn}$ concentration around one atomic percent. This phenomenon of decreasing brightness efficiency as dopant concentration is increased is called concentration quenching.

As mentioned earlier, the hot-electron distribution function is dependent on the concentration of dopants. Therefore, it is reasonable to understand that, at low $\mathrm{Mn}$ concentration, the generation of $\mathrm{EL}$ increases with increasing $\mathrm{Mn}$ concentration because 


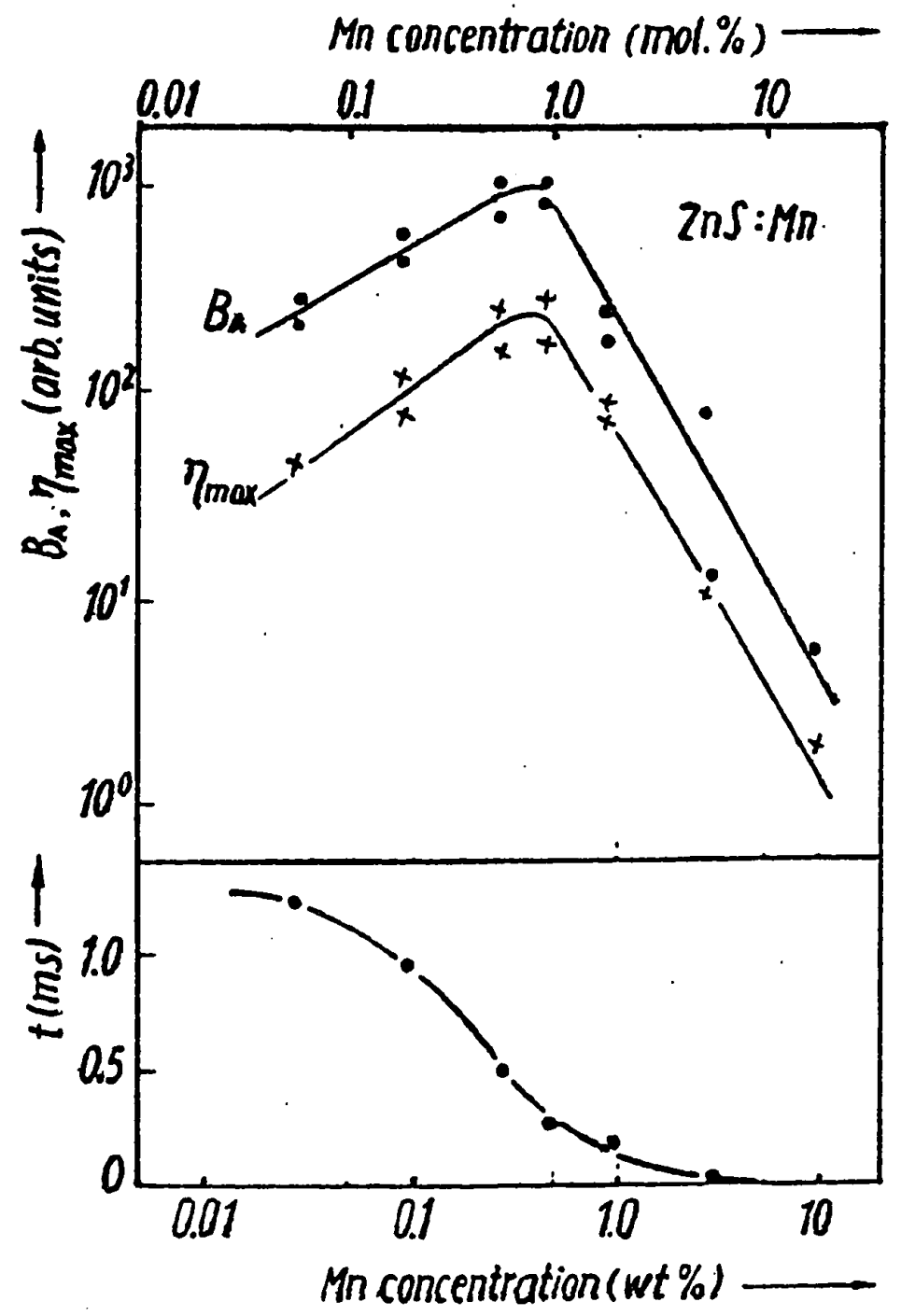

Fig. 4. (a) The brightness efficiency-Mn concentration characteristic and (b) the relationship of decay time v.s. Mn concentration of $\mathrm{ZnS}: \mathrm{Mn}^{(13)}$. 
of the higher probabilities of hot-electron impact excitation on Mn centers. At $\mathrm{Mn}$ concentration over 1 atomic \%, the characteristic yellow emission of $\mathrm{Mn}^{2+}$ centers gradually decreases along with the emergence of red and infra-red emissions(27). The reason for this phenomenon is that, at high $\mathrm{Mn}$ concentration with low level of excitation, excited $\mathrm{Mn}$ centers can resonantly transfer their energies via several unexcited $\mathrm{Mn}^{2+}$ centers, from site to site, to impurity centers which then transfer absorbed energies via either nonradiative channels like heat or red and infra-red radiations(11)(28). Usually, those impurity centers are called "killers" and $\mathrm{Fe}$ ions are known to be such killers in ||$-V \mid$ compounds(29). Therefore, the brightness efficiency of yellow emission starts to decrease at $\mathrm{Mn}$ concentration over 1 atomic \%. In addition, the influence of a nonlinear interaction between $\mathrm{Mn}^{*}$ at high level excitation also opens up another non-radiative channel for concentration quenching. This is due to the more frequent cross-relaxation of $M n^{*}+M n^{*}-->M n+$ $M n^{\star *}$ at high level excitation(29)(30). Therefore, the higher the excitation level, the shorter the decay time becomes. This too can result in the reduction of overall lifetime of $\mathrm{Mn}^{*}$ as seen in Fig. 4 (b) and thus the reduction in the brightness.

The Auger effect (AE), as shown in Fig. 5(b), can be thought of as a reverse process of impact excitation at a luminescent center (Fig. 5(a)). As mentioned earlier, an excited localized-luminescent center will give off its energy and return to ground state after its decay time passes. If this energy is not released radiatively and is 


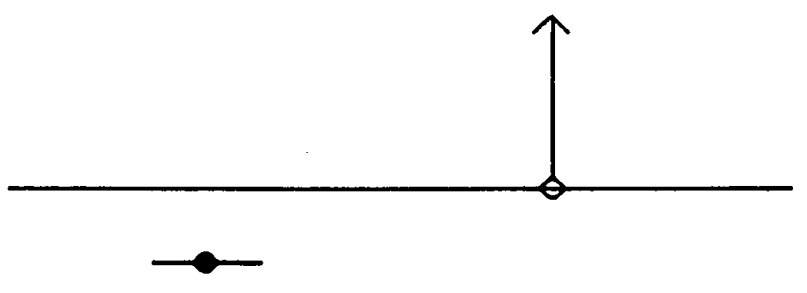

EL

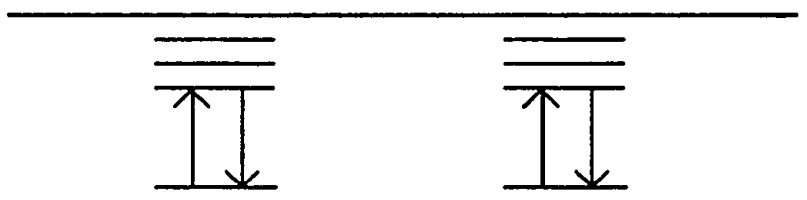

Fig. 5. Schematic illustration of (a) the generation of EL, which is formed as excited luminescent centers return to ground state radiatively; (b) the Auger effect, where the free electron is ejected by the absorption of energy transferred from excited luminescent centers via non-radiative paths.

absorbed by an electron which is near the bottom of the conduction band, this electron can be ejected. This phenomenon is called the Auger effect and it usually occurs in the bulk of the crystal, where lifetime is very short(31). Although not only free carriers, but also weakly bonded donors and acceptors can possibly offer a channel for 
$A E, A E$ due to energy transfer to weakly bound carriers has been found to be much weaker and less likely to occur than free-carrier AE. A concentration of free electrons as low as $10^{12} \mathrm{~cm}^{-3}$ is reported to be able to reduce the luminescence efficiency to $50 \%(32)$.

Besides concentration and Auger quenching, decay time is also a function of temperature. This is due to the overlap of emission and excitation bands at temperature much higher than room temperature (11). This temperature dependence of decay time and brightness of $E L$ devices is usually called thermal, or temperature quenching.

\subsection{Memory Effect and Charge Transfer}

The source of electrons in ac TFEL devices is located at the interface between the phosphor and insulating layers. Those electrons can tunnel into the conduction band of the phosphor under high electric field. By subsequent acceleration in the phosphor layer, some of those energetic electrons can impact-excite the luminescent centers, and $E L$ is then generated after those excited centers return to ground states radiatively. This scenario of $\mathrm{EL}$ generation, sometimes called the field clamping model, has been discussed earlier in a previous section. As Fig. 1 shows, the structure of ac TFEL devices consists of a phosphor layer sandwiched between two insulating layers. Therefore, the whole device can be seen as three capacitors connected in series. Because 
of the capacitance of these two insulating layers, the amount of charge flowing through the phosphor is limited and is controlled by the applied field. Insulating layers must be able to retain this charge stored at the interfaces to prevent the phosphor layer from electrical breakdown. This charge storage results in the so-called memory effect of ac TFEL devices. This hysteretic property has a potential advantage of being used to control the contrast of EL in order to meet the need for a grey scale(33).

After Inoguchi first interpreted a possible reason for hysteresis(33), several studies were performed in order to get a better picture of this effect(34)(35). Band bending, the effect of negative resistance, is believed to be the main cause of hysteresis of ac TFEL devices. The phenomenon of band bending in dielectric films has been widely known due to numerous investigations on the dielectric breakdown of thin $\mathrm{SiO}_{2}$ films in $\mathrm{MOS}^{(36)(37)(38)}$. The band bending, or curvature of the energy bands, can be described using Poisson's equation,

$$
œ x\left(d V^{2} / d X^{2}\right)=Q_{S}
$$

which implies that curvature, the second derivative of voltage $V$, is dependent on the space charge $Q_{S}$. $œ$ is the permittivity. The buildup of trapped-positive charge at the cathode interfaces results in a localized field enhancement, which increases the slope of 
conduction band (Eq. 5) and leads to further injection of tunneling electrons from the interface.

A similar situation exists in ac TFEL devices. During the application of voltage, hot electrons, after tunneling into the phosphor layer, with lower energy can impact and excite luminescent centers, and those with higher energy can impact and ionize either deep donors or the host lattice. As Fig. 2 shows, positive-space charge formed by the impact of hot electrons will be trapped and will distort the conduction band of, for example, $\mathrm{ZnS}$, which further enhances the field at the cathode interfaces. When the applied voltage decreases, the charge stored at the interfaces adds to the internal field to enhance the current flow even though the applied voltage is below threshold. It is this addition of stored charge during reversal of power application which causes bistability, the hysteresis effect, of ac TFEL devices. Fig. 6(a) shows a typical luminance-voltage characteristic illustrating this effect. Holes created from the generation of electron-hole pairs by impact ionization of the ZnS lattice ${ }^{(34)}$, holes from impact-ionization of scattering centers like deep donors and impurity states ${ }^{(35)}$, and the depletion of trapped electrons after being injected from the $\mathrm{ZnS}$ layer into insulating layers ${ }^{(39)}$ are the major models proposed to explain the source of positive-space charge.

Based on known theories, the behavior of charge transfer and charge storage in ac TFEL devices can be further described mathematically. Fig. 6(b) shows a side view of the structure of an ac 


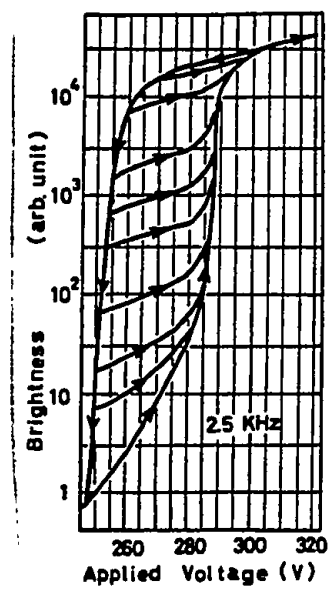

(a)

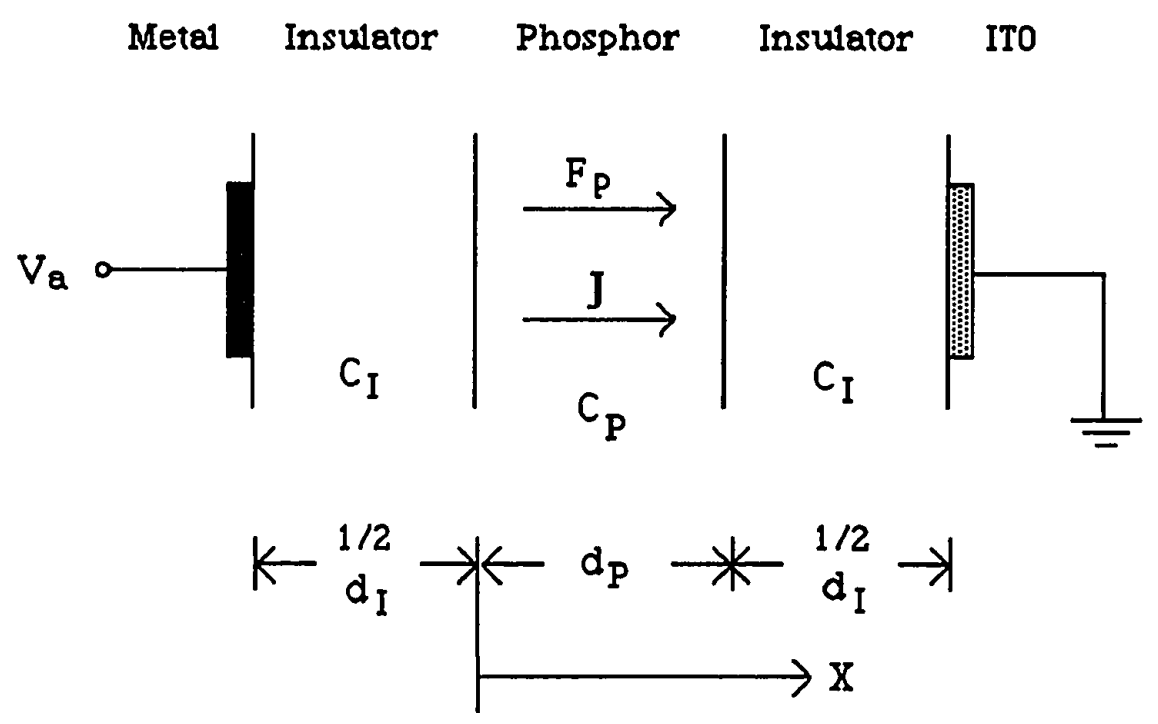

(b)

Fig. 6. (a) The hysteresis effect of the ac TFEL devices ${ }^{(33)}$, and (b) a side view of an ac TFEL device using double-insulating-layer structure. 
TFEL device and its equivalent circuit. Index 1 refers to the insulating layer and $P$ to phosphor layer. Externally applied voltage is denoted by $V_{a}$. If, in the beginning, there is no space charge in the bulk and insulators, $Q_{a}$, the surface charge per unit area at the external anodic electrode is given by

$$
Q_{a}=Q_{c}+C_{p} \times d_{p} \times F_{p}
$$

where $Q_{c}$ is the surface charge per unit area at the cathodic interfaces between insulator and phosphor layer, $F_{p}$ is the electric field in the phosphor, and $C_{p}$ and $d_{p}$ are the capacitance and thickness of the phosphor layer, respectively. The second term in Eq. 6 is the dielectric displacement in the phosphor. The relation among $V_{a}, F_{p}$ and $F_{1}$, the electric field in the insulators, can also be written as

$$
\begin{aligned}
V_{a}= & F_{1} \times\left(d_{1} / 2\right)+d_{p} \times F_{p}+F_{1} \times\left(d_{1} / 2\right) \\
& =F_{1} \times d_{1}+d_{p} \times F_{p}
\end{aligned}
$$

where $d_{1}$ is the thickness of the insulating layers. By adding $Q_{a}=C_{1} \times$ $d_{1} \times F_{1}$ to Eq. 6 and equating $\left(E q .7 \times C_{1}\right)-$ Eq. 6, we have

$$
\left(C_{1} \times V_{a}-Q_{c}\right)=\left(C_{1}+C_{p}\right) \times F_{p} \times d_{p}
$$

which may be rearranged to give 


$$
F_{p}=\left(C_{1} \times V_{a}\right) /\left(C_{1}+C_{p}\right) \times d_{p}-Q_{c} /\left(C_{1}+C_{p}\right) \times d_{p}
$$

Recalling Eq.1 and Eq.2 from section 2.3, we know that tunneling current due to the states with energy between $E$ and $E+d E$ is

$$
d J=q \times f(E) \times N(E) \times e_{n} \times d E
$$

After integrating Eq. (9) from $E_{c}$ to $E^{\star}$, the tunneling current $J$ comes out to be

$$
J=(2 / 3) \times\left(q \times N_{s}\left(E^{*}\right) /\left(E_{c}-E^{*}\right)^{1 / 2}\right) \times K \times F \times e_{n}\left(E^{*}\right)
$$

where $E^{*}$ is a pseudo-Fermi level upon which $f(E)=0$ and below which $f(E)=1, E_{c}$ is the energy at the bottom of the conduction band, $N_{s}$ is the density of surface states, and $\mathrm{K}$ is the first term of Eq. 1. Since $d Q_{c} / d t=J$, by substituting Eq. 10 into Eq. 8 and taking the derivative on both sides of Eq. (8) with respect to time $t$, we get the first derivative of the field, $F_{p}$, to be

$$
\begin{aligned}
d F_{p} / d t= & \left(1 /\left(C_{1}+C_{p}\right) \times d_{p}\right) \times\left(C_{1} \times d V_{a} / d t-\right. \\
& \left.\left(q \times N_{s}\left(E^{*}\right) /\left(E_{c}-E^{*}\right)^{1 / 2}\right) \times B \times F \times e_{n}\left(E^{*}\right)\right)
\end{aligned}
$$

Eq. 8 and 11 show that the electric field in the phosphor layer is reduced by the storage of charge $Q_{c}$ in the cathode interface, which causes the hysteresis effect of ac TFEL devices. This reduction of electric field in the phosphor layer prevents the ease of dielectric 
breakdown so that the stability and lifetime of TFEL devices can be promoted. It is obvious that, from Eq. 8 and 11, thick phosphor layer are preferred for reducing the field in the phosphor. Details on the requirements and choice of insulating layers will be discussed in the next section. The solutions of Eq. 11, based on different distributions of traps and excitation waveforms, have been proposed and are available in references 14 and 19. If electron multiplication is taken into account and is not necessarily equal to one, Eq. 11 can be modified by adding a multiple $M(F)$, the multiplication factor, to the second term of Eq. 11. Details are also available in another paper by $E$. Bringuier(40).

The hysteresis effect in $\mathrm{ZnS}: \mathrm{Mn}$ becomes more pronounced as the $\mathrm{Mn}$ concentration is incerased above 1 atomic \%(41). However, concentration quenching will dominate to reduce the intensity of EL at high $\mathrm{Mn}$ concentration. A tradeoff between brightness and hysteresis of ac TFEL devices seems unavoidable.

\subsection{Summary}

High brightness and long life ac TFEL devices have been successfully made using the MISIM structure. The studies of EL generation for commercial use are mainly focused on $\mathrm{ZnS}: \mathrm{Mn}$ due to its high efficiency and good brightness. EL generation, as well as phenomena like concentration quenching and the memory effect which occurs in ZnS:Mn TFEL devices, can be well explained by 
combining impact excitation, energy transfer and positive-space charge accumulation models.

According to these models, EL in $\mathrm{ZnS}: \mathrm{Mn}$ is generated after excited $\mathrm{Mn}^{2+}$ centers, which are formed by the impact excitation of $\mathrm{Mn}^{2+}$ by tunneling electrons, return to ground states and release the gained energy radiatively. At low $\mathrm{Mn}$ concentration, the generation of luminescence increases with increasing $\mathrm{Mn}$ concentration. At concentrations of Mn over 1 atomic \%, the channel of resonant energy transfer from excited $\mathrm{Mn}^{2+}$ via unexcited $\mathrm{Mn}^{2+}$ to killers, where energy releases nonradiatively, gradually dominates over the regular radiation channel. This concentration quenching causes the decrease of decay time of excited centers and the brightness of luminance released.

The purpose of insulating layers is to limit the amount of charge flowing through the phosphor in order to avoid damage of the devices. Positive-space charge stored in insulating layers causes distortion of the conduction band of $\mathrm{ZnS}$, which, in turn, enhances the field at cathode interfaces. As applied voltage reverses, stored charge adds to the internal field and the bistability of ac TFEL devices emerges. The relations of electric field in the phosphor layer $F_{a}$, storage charge $Q_{c}$, tunneling current $J$, and applied voltage $\mathrm{V}_{\mathrm{a}}$ can be expressed mathematically using Eq. (8) or Eq. (11), which imply that thick phosphor layers are preferred. 


\section{Choice of Materials and Fabrication Technologies}

\subsection{Phosphor Layer}

$\mathrm{ZnS}$ is the preferred choice of host for ac TFEL devices because of its wide band gap, ${ }^{(42)}$ and ac TFEL devices using $\mathrm{ZnS}: \mathrm{Mn}$ as the phosphor layer have been found to have the best brightness. The use of different centers other than $\mathrm{Mn}$ to generate $\mathrm{EL}$ with different color has been widely tested in ZnS. Table 1 lists some of them. It is known that a combination of RGB--red, green and blue light--with suitable brightness balance and contrast ratio can display a full range of colors. Both green and red EL with good brightness have been obtained from $\mathrm{ZnS}: \mathrm{TbF}_{3}$ and $\mathrm{ZnS}: \mathrm{SmF}_{3}$, respectively. However, the use of $\mathrm{ZnS}$ as a host material to generate blue EL suffers from low brightness. ZnS:Tm is a known blue phosphor. Although the percentage of blue is only $10 \%$ of the total white brightness $(25 \%$ red and $65 \%$ green), ${ }^{(43)}$ the brightness of blue, compared with that of red and green in Table 1, is still too low to be commercially used. Therefore, to pursue full color display, or more precisely, to find a suitable blue phosphor, has become the driving force of searching for other host materials.

The first requirement of the host is that its energy of band gap must be larger than that of the desired optical transition. The highest transition energy of visible light, blue emission, is $2.8 \mathrm{eV}$. If 


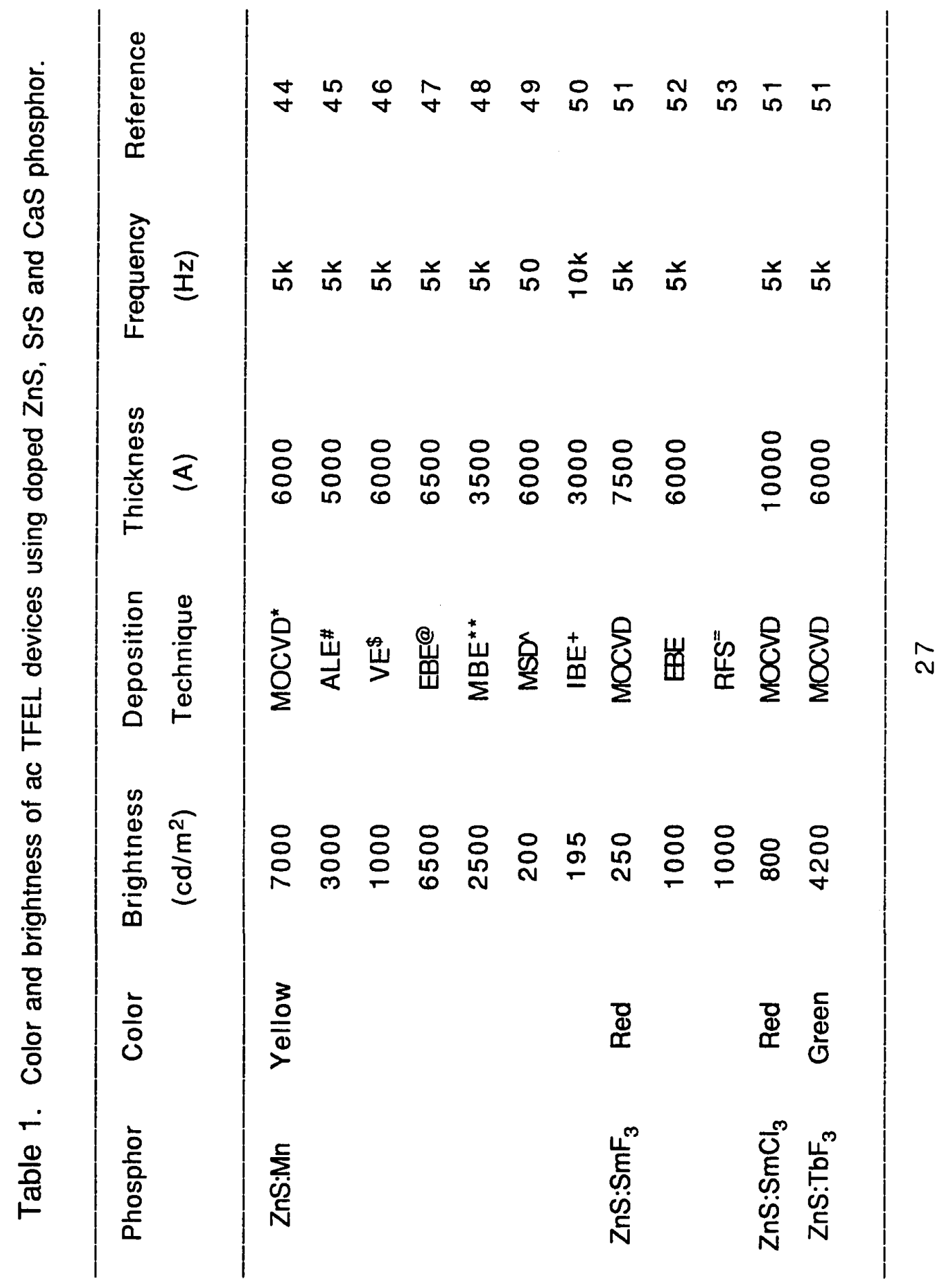




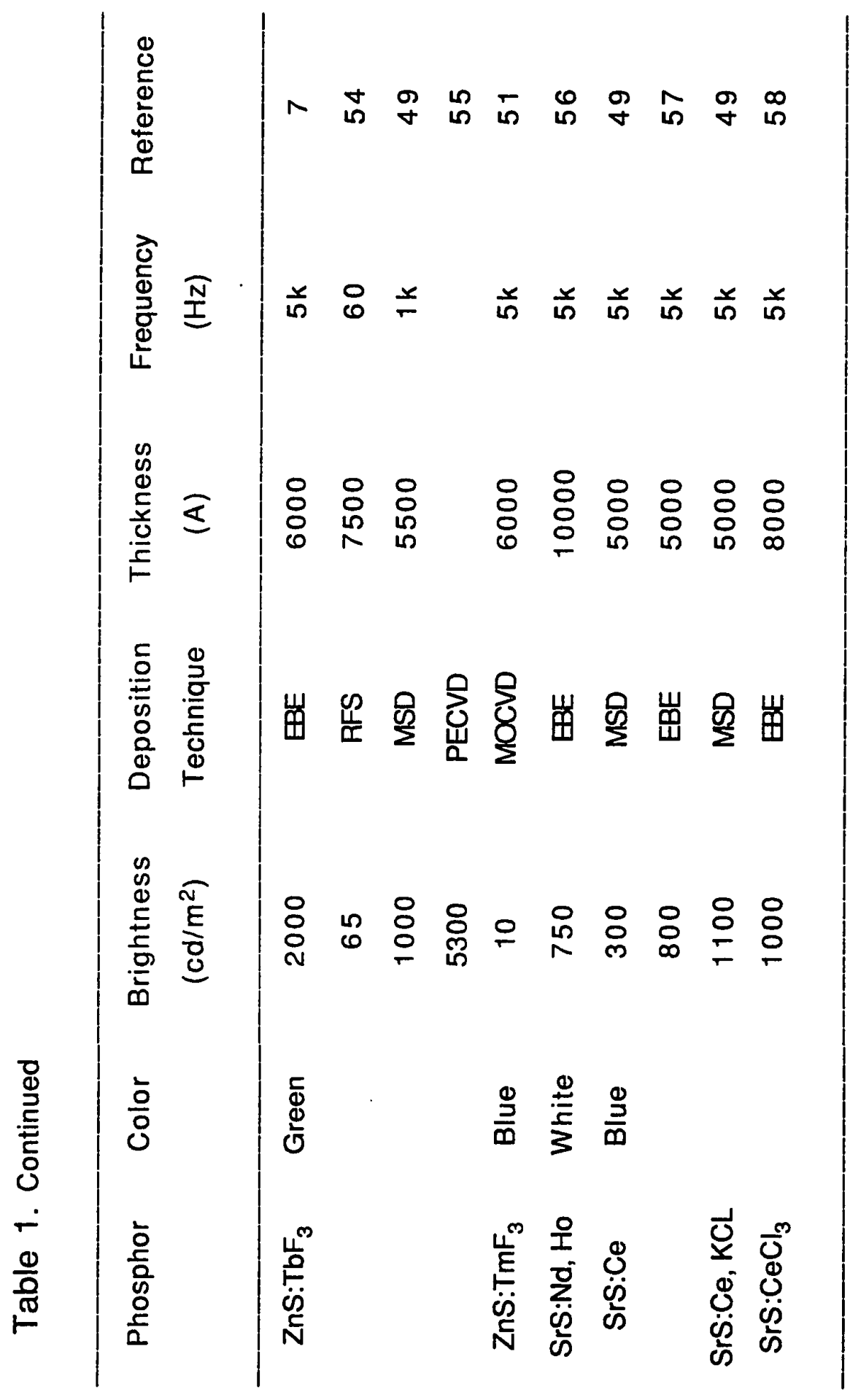

$\infty$ 


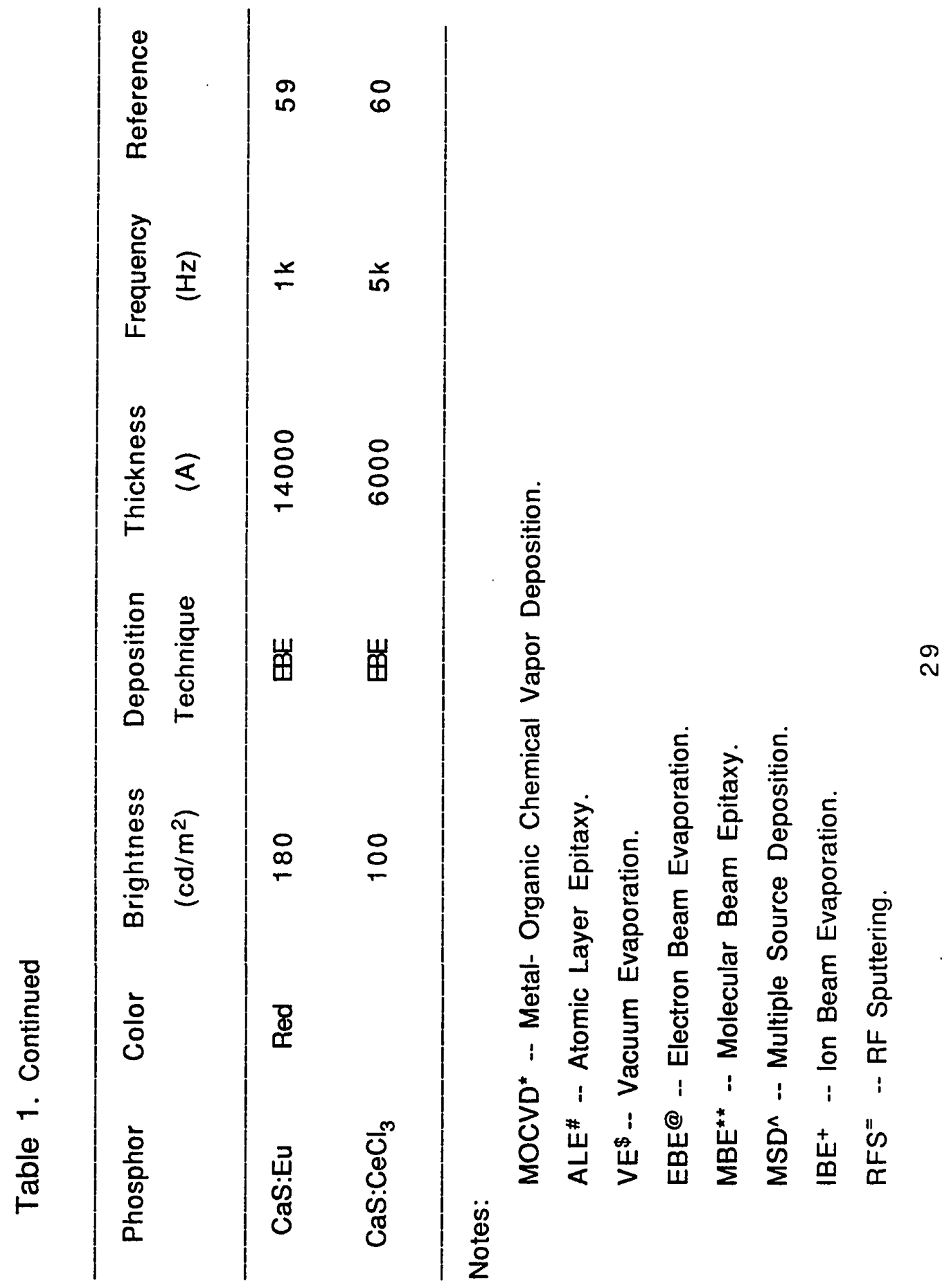


the energy difference between excitation and emission is $0.5 \mathrm{eV},(13)$ the minimum band gap needed for a host to cover the entire visible region then comes out to be $3.3 \mathrm{eV}$. ZnS with the gap of $3.66 \mathrm{eV}$ is just about right to be the host for the emission of full color when doped with different centers. Other than $\mathrm{ZnS}, \mathrm{SrS}$, and CaS are also candidates with the potential for full-color TFEL displays because of their wide band gaps. The band gaps of those host materials are listed in Table 2. Among them, SrS attracts much attention. SrS:Ce is now the phosphor known to generate the brightest blue EL, 650 $\mathrm{cd} / \mathrm{m}^{2}$. Although the brightness of red $\mathrm{EL}$ of $\mathrm{ZnS}: \mathrm{SmF}_{3}$ is acceptable, its poor chromaticity pushes researchers to find other candidates. $\mathrm{CaS}: \mathrm{Eu}$ is one of the major contenders for red EL applications.

Table 2. The energy gap of some host materials usually used.

\begin{tabular}{lcc}
$\begin{array}{c}\text { Materials } \\
(\mathrm{eV})\end{array}$ & Energy gap & Reference \\
\hline ZnS & 3.66 & 10 \\
ZnSe & 2.7 & 60 \\
SrS & 4.3 & 62 \\
CaS & 4.4 & 63 \\
CoS & 2.5 & 64 \\
\hline
\end{tabular}


Other requirements on host materials are mainly concentrated on the performance of some operating parameters like power and transparency. The long time problem of the relatively high power needed for $\mathrm{ZnS}$ can be dramatically reduced by using $\mathrm{ZnSe} ; \mathrm{ZnF}_{2}$ can reduce internal light trapping, a significant effect in $\mathrm{ZnS}$, due to its lower refractive index. However, these materials suffer from low efficiency and brightness. Commercialization is far less than for ZnS.

We know that impact excitation of luminescent centers by tunneling electrons is the main mechanism of EL generation. EL is generated after those excited centers return to ground states radiatively. Thus, better brightness of EL might be achieved if one or more of those mechanisms involved would react efficiently. Efficient acceleration of tunneling electrons requires large mean free paths or good crystallinity of host. Therefore, a host with large grains will be preferred for increasing the efficiency of accelerating hot electrons. However, the size of grains is more deposition-technique dependent. The deposition-technology dependence of phosphor layers will be discussed in section 3.4 and 3.5. 


\subsection{Dopants}

The detailed mechanisms of generating EL have been discussed in section 2, where $E L$ is generated as excited luminescent centers returning to ground states. The purpose of adding dopants into the host is to introduce luminescent centers for hot-electron impact excitation. The energy difference between the excited and ground states of a luminescent center determines the wavelength of radiation released. This gives us a way to control the colors of EL by simply choosing a suitable dopant for a given host. A wide variety of colors have been demonstrated using different dopants in those wide band-gap hosts mentioned earlier and listed in Table 1.

The requirements for suitable dopants are mainly based on our understanding of EL generation theories. As mentioned in the previous section, the excitation rate of luminescent centers after electron impact, which can be expressed using Eq. 5, is a function of the velocity of tunneling electrons, the hot-electron distribution function, and the impact cross section of luminescent centers. The way to increase the energy of hot electrons has been pointed out in the previous section. The first requirement on a suitable dopant is

then that it should be able to provide a large cross section for impact excitation by hot electrons to increase the excitation rate. Because hot electron impact on isolated centers is more efficient than that on clusters, high solubility of dopants into host material is also required for efficient hot-electron impact. In order to 
convert absorbed energy into EL efficiently, the dopant chosen should have as little non-radiative energy transfer like concentration quenching and Auger quenching as possible. Finally, of course, the wavelength of emission has to be in the range of visible light of the color wanted. Chromaticity is also concerned, especially for full color display.

$\mathrm{ZnS}: \mathrm{Mn}$ is now the only phosphor producing $\mathrm{EL}$ with brightness reaching commercial needs. Its ${ }^{4} T_{1}$ to ${ }^{6} A_{1}$ transition gives off yellow-orange luminance with wavelength at $585 \mathrm{~nm}(2.12 \mathrm{eV})$. In addition to $\mathrm{Mn}$, rare earth elements are widely chosen as the dopants for hosts like $\mathrm{ZnS}, \mathrm{SrS}$ and CaS. The transitions involved are between well shielded $4 f$ inner shell states and $4 f$ ground states. Thus rare earth dopants promise to offer a wide variety of colors. Those usually used rare earths with different hosts are listed in Table 1 as well.

As far as we know, only the trivalent rare earths are of practical use in ac TFEL devices. This might be due to the instability of other states like the divalent state, where the radiative transition involved is from not well shielded $4 f$ to $5 d$.(13) Among those rare earth elements, only Tb doped $\mathrm{ZnS}$ produces green luminance with the brightness close to that of $\mathrm{ZnS}: \mathrm{Mn}$ (see Table 1). There are totally seven possible paths of transition in $\mathrm{ZnS}: \mathrm{TbF}_{3}{ }^{\left({ }^{(65)}\right.}$ The one which involves the ${ }^{5} D_{4}$ to ${ }^{7} F_{5}$ transition gives off the most intensive emission with a wavelength around $540 \mathrm{~nm} .{ }^{(65)}$ 
The reason why rare earths are inferior in brightness may be that one or more of those requirements for suitable dopants discussed earlier is or are missing. More complicated energytransfer processes of rare earths than that of $\mathrm{Mn}$ in ac TFEL devices might be the main reason for reduced brightness. Besides the regular use of dopants in the host, the addition of small amount of codopants like $\mathrm{Cl}$ in $\mathrm{ZnS}: \mathrm{Mn}$ can improve the brightness of luminance and lower the operating voltage(16).

\subsection{Color EL Displays}

ZnS:Mn ac TFEL displays are now commercialized. The proposed lifetime can be as long as that of the CRT. The development of full color EL displays is now under way. In addition to the demonstrated success of green light from ZnS:Tb, the development of a red phosphor, ZnS:Sm, is now on the edge of achieving the final goal. Most of the efforts are devoted on finding a suitable bright blue. There are five possible candidates for blue phosphors: ZnS:Tm, $\mathrm{SrS}: \mathrm{Ce}, \mathrm{La}_{2} \mathrm{O}_{2} \mathrm{~S}: \mathrm{Tm}, \mathrm{CaS}: \mathrm{Bi}$ and $\mathrm{CaS}: \mathrm{Cu}$. ${ }^{(66)}$ Although some of them exhibit good stability and chromaticity, the best blue brightness is still $30 \%$ less than what is required. (42) People try to apply different fabrication techniques to compensate for the difference. There are mainly four possible approaches to color EL displays: the patterned phosphor structure, where full color pixel is formed from adjacent RGB phosphor stripes ${ }^{(67)}$; the stacked structure, which uses 


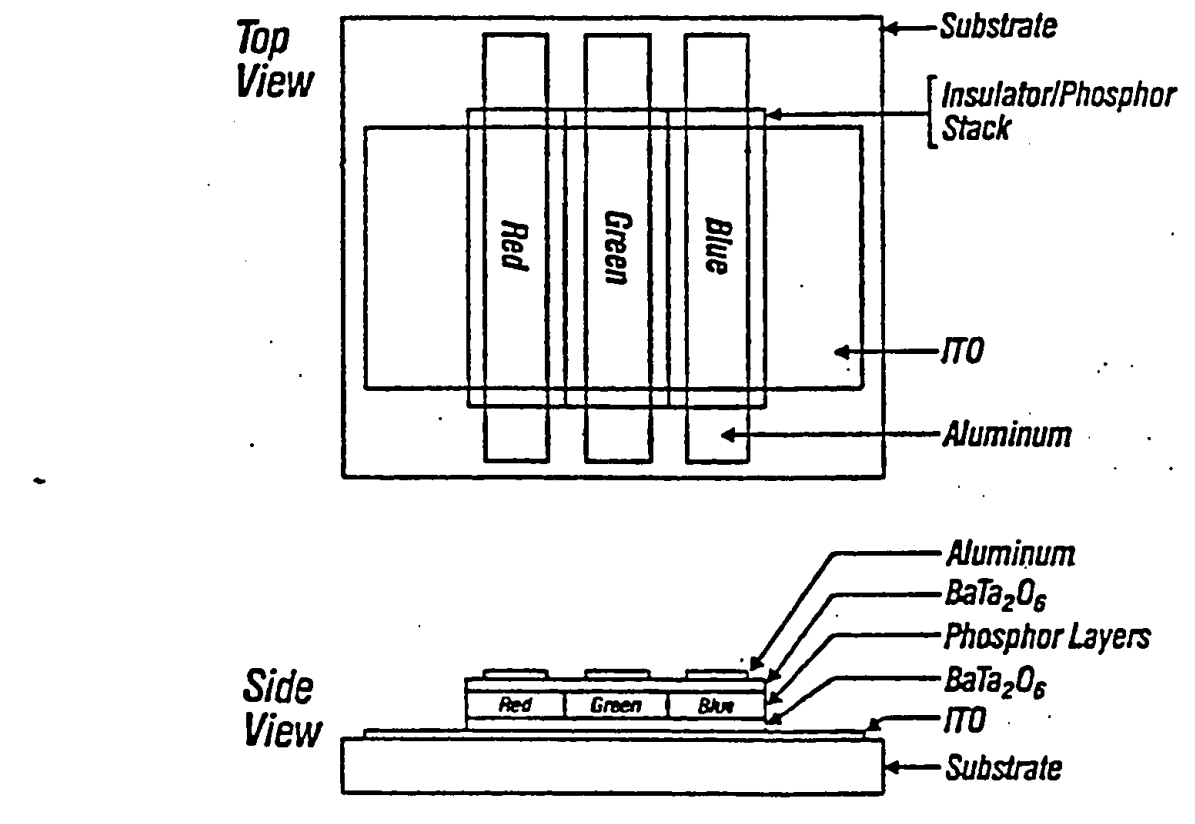

(a)

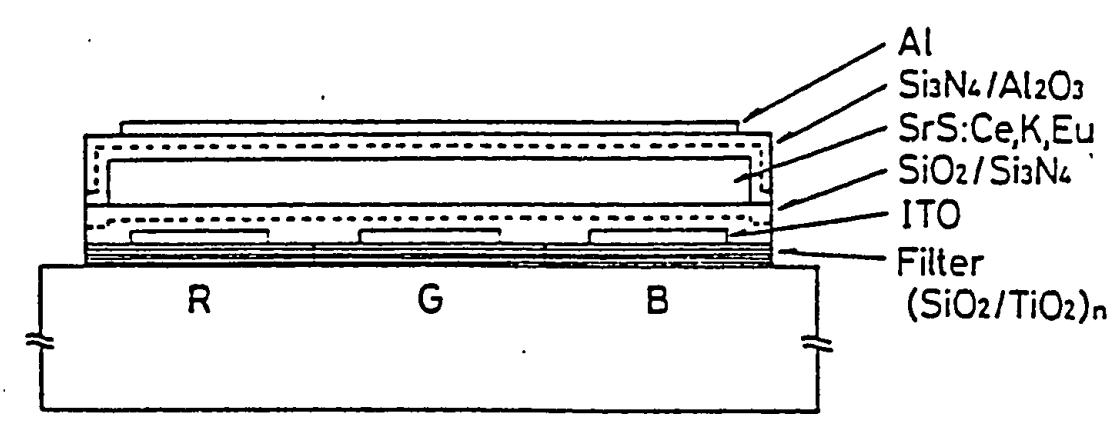

(b)

Fig. 7. The schematic illustrations of (a) the patterned phosphor structure ${ }^{(67)}$, and (b) the patterned filter method. ${ }^{(70)}$ 
separated RGB phosphor layers(68); the hybrid structure, which combines both patterned and stacked structures along with color filters to produce full colors ${ }^{69)}$; and the patterned filter method, which uses RGB filters over a white phosphor layer to generate full colors. ${ }^{(70)}$ Among them, the patterned phosphor structure and the patterned filter method seem to offer the best promise. The schematic diagrams of these two structures are shown in Fig. 7. $\mathrm{ZnS}: \mathrm{Sm}, \mathrm{ZnS}: \mathrm{Tb}$ and SrS:Ce are used as RGB phosphors in the patterned phosphor structure. White phosphor from SrS:Ce, Eu and $K$ is used in the patterned filter method. The advantage of the patterned phosphor structure is the ease of using standard electrodes. However, it suffers from a lower than average brightness due to the small pixel fill factor. The major drawback of the latter approach is that a lot of light generated will be lost in the filters.

\subsection{Fabrication Technologies of $\mathrm{ZnS}: \mathrm{Mn}$}

Although the first ac EL device was made by Destriau in 1936 using $\mathrm{ZnS}: \mathrm{Cu}$ powder as phosphor, $(4)$ the development of this technology has been very slow. This slow progress is mainly due to the lack of a theoretical understanding of II-VI compounds. Research and development of EL displays can be divided into two major periods. During the first period, from the early 1950 s to the middle 1960s, EL fabrication and research were mainly based on powder- 
type ac EL devices. Because problems like insufficient brightness, instability, low lifetime, and poor contrast ratio due to the scattering of light by the powder-phosphor layer seemed unable to be overcome at that time, the first period of development of EL displays then gradually came to an end. The second period of research of thin ZnS:Mn films as phosphor began in 1960. Vacuum evaporation and conventional chemical vapor deposition (CVD) were the methods for preparing thin films in the 1960s. But no devices with acceptable lifetime for commercial use were produced (Typical lifetimes were from a few minutes to hours only.). It was not until 1974 that the first long lifetime and good brightness ac TFEL devices were made by the team at Sharp.(3) In their studies, thin $\mathrm{ZnS}: \mathrm{Mn}$ films were prepared by E-beam evaporation. Brightness of $3420 \mathrm{~cd} / \mathrm{m}^{2}$ and more than $10^{4}$ hours of operating time were achieved by Sharp, et al. This improvement, from the technical point of view, can be attributed to the following developments: (1) the replacement of the poor transparent conductor, tin oxide, by indiumtin-oxide (ITO), which provides better uniformity and a smooth layer; (2) the use of an MISIM structure with the choice of better insulating material, which allows devices to be operated under a stable and sufficiently high voltage; and (3) the use of better deposition technology, which excludes the contaminations of moisture, oxygen and mobile ions such as $\mathrm{Cu}^{+}$and $\mathrm{Fe}^{2+}$ in the phosphor layer. 
Since Inoguchi's work(3), the development of EL displays then went into the second period. This development has spread into two different approaches: the DCEL displays and the ac TFEL displays. DC type EL displays, which use thick phosphor films, are fundamentally resistive-type devices. Although DC type EL displays are easier and cheaper to make, their long term stability is still a problem. The advantages of stability, high brightness and memory effect in AC type EL displays resulted in ac TFEL displays not only superior to the DC type, but also the most promising for making flat-panel displays.

At present, there are several applications of ac TFEL displays in the commercial market and in defense. The techniques employed to deposit $\mathrm{ZnS}: \mathrm{Mn}$ also have expanded from evaporation and CVD into spray and other processes which have been widely used in the semiconductor industry, such as if sputtering, atomic layer epitaxy (ALE), and metal-organic chemical vapor deposition (MOCVD). The disadvantages of using evaporation and conventional CVD to deposit ZnS and other host materials are the driving force for pursuing alternative technologies. The performance of $E L$ is very dependent on the process chosen. From the point of view of the requirements for phosphors mentioned in the previous sections, and in order to increase lifetime, brightness and stability of ac TFEL devices, an ideal deposition technology should have the ability to make a phosphor layer with precise stoichiometry, desired crystallinity and orientation, minimum contamination from moisture and mobile ions, 
high index of refraction, high throughput and the potential to make large area depositions with good uniformity.

Evaporation This is the earliest and simplest method to deposit ZnS:Mn. Vacuum evaporation and electron-beam evaporation (EBE) are the two major evaporation techniques. In most cases, phosphor films deposited using those methods would be followed with thermal annealing for several hours. The problems of using single source evaporations are the unavoidable introduction of impurities and the difficulty of crystallinity and stoichiometry control. The origin of those impurities may come from: the interaction of source with container, contamination from oxygen and organic traces, and the dissociation of the phosphor, e.g. $\mathrm{ZnS} \mathrm{-->} \mathrm{Zn+}$ $1 / 2 \quad S_{2} \cdot(71)$ This dissociation and possible recombination of evaporated components would influence the control of stoichiometry as well as the crystallinity and the orientation of phosphors. Furthermore, the interaction of various dielectric layers with host materials like $\mathrm{ZnS}$ also limits the applications of evaporation. A modification on vacuum evaporation and EBE is made possible by using multiple source evaporation (MSE). The stoichiometry of evaporated $\mathrm{ZnS}$ and $\mathrm{Mn}$ concentration in $\mathrm{ZnS}$ can be well controlled using three separated evaporation sources, $\mathrm{Zn}, \mathrm{S}$, and $\mathrm{Mn}$. Uniform film growth can be obtained as well. Ion beam evaporation (IBE) is another way of depositing phosphor films. ZnS:Mn layers can be deposited using $\mathrm{Zn}, \mathrm{S}$ and $\mathrm{Mn}$ ions. Those ions come from a highdensity and high-temperature plasma which is generated by the 
bombardment of a $\mathrm{ZnS}: \mathrm{Mn}$ target by an intense ion beam. ${ }^{(50)}$ But the maximum brightness from IBE deposited $\mathrm{ZnS}: \mathrm{Mn}$ is too low to be used. (See Table 1.)

Sputtering This process is widely used in the semiconductor industry to deposit metallic and insulating films. ZnS can also be deposited using this technique, especially by if sputtering. High temperature annealing $\left(450-550^{\circ} \mathrm{C}\right)$ after film deposition is recommended in order to get good EL performance. The performance of ac TFEL devices prepared by if sputtering has been proven to be better than that of devices prepared by evaporation. (72) However, the difficulties of using if sputtering for large area depositions, and of controlling the stoichiometry and crystallinity of deposited films, hinder its application for display manufacture. Using reactive sputtering to compensate for the loss of sulphur may improve the latter problem, but the limitation of the former problem seems hard to overcome.

Atomic layer epitaxy This process is carried out by a sequence of alternate deposition reactions of elements. These elements can be introduced either as the elemental vapors or as vapor compounds of the elements. For example, deposition of $\mathrm{ZnS}$ can be achieved by either using elemental vapors of $\mathrm{Zn}$ and $\mathrm{S}$ or $\mathrm{ZnCl}_{2}$ and $\mathrm{H}_{2} \mathrm{~S}$ vapor compounds ${ }^{(73)}$. In principle, the growth of ALE relies on the difference between chemisorption and physisorption. This can be achieved by the adjustment of suitable temperature, which is controlled to let only one monolayer of elements chemisorb on the 
surface of substrate. A single layer of ALE $\mathrm{ZnS}$ is obtained when a first atomic layer of $\mathrm{Zn}$ and a second atomic layer of $\mathrm{S}$ are deposited successively onto the substrate surface, where $\mathrm{Zn}-\mathrm{S}$ bonding occurs. Thus, the growth rate of ALE is more temperature rather than pressure dependent. This deposition sequence of $\mathrm{Zn}$ and $\mathrm{S}$ is continued until the desired thickness is reached. The performance of ALE deposited ZnS in ac TFEL devices has been proven to be superior to that of films deposited by other methods. ${ }^{(16)}$ This advantage may come from the better structure and stoichiometry of ALE grown ZnS films due to their well controlled growth. Large area deposition is also available in ALE. However, this technique is an extremely timeconsuming process. The low deposition rate, about $0.1 \mu \mathrm{m} / \mathrm{hr}^{(16)}$, becomes the major drawback of using ALE. High cost is inevitable.

CVD This was one of the earliest methods used to prepare ac TFEL devices. Thin ZnS films were grown using zinc chloride with $\mathrm{H}_{2} \mathrm{~S}$ in the 1960s. The problem of using CVD prepared $\mathrm{ZnS}$ was its poor film uniformity and short lifetime. This short lifetime is due to the ease of dielectric breakdown of $\mathrm{ZnS}$ by the introduction of halide residues in $\mathrm{ZnS}$ during deposition. This problem was not solved until an organic zinc compound was introduced to the CVD process. This process of using metal-organic compounds is called metal-organic chemical vapor deposition (MOCVD). Besides, the preparation of conventional CVD ZnS films has been improved to use a new approach called spray pyrolysis. 
Spray Pyrolysis The pyrolysis process, sometimes called vapor phase pyrolysis, is now being examined by many researchers and is one of the promising technologies to obtain good ZnS films ${ }^{(74)}$. Essentially, the principle of this method is to directly spray gastype materials onto the surface of a heated substrate, where those materials decompose into the desired compound or element. In order to decompose efficiently and easily, chelate compounds are the best choice for preparing thin films using spray pyrolysis. Metal-organic chelates like zinc diethyldithocarbamate, $\mathrm{Zn}\left(\left(\mathrm{C}_{2} \mathrm{H}_{5}\right)_{2} \mathrm{NCS}_{2}\right)_{2}$, and zinc xanthate, $\mathrm{Zn}\left(\left(\mathrm{CH}_{2} \mathrm{O}\right) \mathrm{CS}_{2}\right)_{2}$, with carrier gas, $\mathrm{N}_{2}$, are usually used to deposit $\mathrm{ZnS}$.(75) The decomposition equation of zinc diethyldithocarbamate can be written as

$$
\mathrm{Zn}\left(\left(\mathrm{C}_{2} \mathrm{H}_{5}\right)_{2} \mathrm{NCS}_{2}\right)_{2(\mathrm{~g})} \stackrel{400^{\circ} \mathrm{C}}{\mathrm{N}_{2}} \mathrm{ZnS}_{(\mathrm{S})}+\left(\left(\mathrm{C}_{2} \mathrm{H}_{5}\right)_{2} \mathrm{NCS}\right)_{2} \mathrm{~S}_{(g)}
$$

A deposited film with good control of uniformity, stoichiometry, dopant amount and crystallinity may be expected by using the spray method. Another advantage of spray pyrolysis is that not only phosphor layers, but also all thin films required for EL devices, such as ITO, insulating layers and metallic conductors, can be made using this tecinnique ${ }^{(75)}$.

Although vacuum evaporation and EBE are still the major techniques used to deposit phosphor layers, they will be gradually replaced by new methods which meet the stringent requirements for 
the fabrication of large-area ac TFEL displays. ALE prepared phosphor films perform well but are costly. MOCVD and spray techniques seem to offer better promise for the deposition of phosphor layers for ac TFEL devices.

\subsection{MOCVD ZnS Layer}

MOCVD has been widely used in the semiconductor industry for the deposition of pure epitaxial films like tungsten. MOCVD ZnS was first prepared by Manasevit using bubbled diethyl zinc, $\left(\mathrm{CH}_{3}\right)_{2} \mathrm{Zn}$, with hydrogen sulfide, $\mathrm{H}_{2} \mathrm{~S}$, in 1971.(76) The driving force of pursuing this technique is due to the promise of low deposition temperature, good stoichiometry and better dopant control along with the potential of large area deposition. Furthermore, if suitable methods during deposition can be carried out, post deposition annealing may not be necessary.

As described in the previous section, the use of metal-organic sources to deposit phosphor layers such as $\mathrm{ZnS}$ can be approached in two different ways: the additive method and the decomposition method. The former is what we usually call MOCVD; the latter is what has been discussed in the previous section as spray pyrolysis. Both of these two approaches use metal-organic (MO) sources to deposit $\mathrm{ZnS}$. The major difference between them is that the former uses two different reactive gases to form $\mathrm{ZnS}$ on the heated 
substrate, where chemical reactions take place; the latter typically uses zinc-organic chelates like $\mathrm{Zn}\left(\left(\mathrm{C}_{2} \mathrm{H}_{5}\right)_{2} \mathrm{NCS}_{2}\right)_{2}$ to form $\mathrm{ZnS}$ after the chelates decompose on the heated substrate (Eq. 12). Dimethyl zinc with hydrogen sulfide are the chemicals widely used for depositing MOCVD ZnS.(51)(77)(78)(79) The chemical reaction of dimethyl zinc (DMZ) with hydrogen sulfide, $\mathrm{H}_{2} \mathrm{~S}$, can be expressed as

$$
\left(\mathrm{CH}_{3}\right)_{2} \mathrm{Zn}_{(\mathrm{g})}+\mathrm{H}_{2} \mathrm{~S}_{(\mathrm{g})} \underset{\text { Heat }}{-\cdots--->} \mathrm{ZnS}_{(\mathrm{S})}+2 \mathrm{CH}_{4(\mathrm{~g})}
$$

This reaction can be carried out at both atmosphere pressure ${ }^{(80)}$ and in low pressure systems. ${ }^{(81)}$ Other than $D M Z$ and $\mathrm{H}_{2} \mathrm{~S}$, gases like diethyl zinc(81)(82) for the sources of $\mathrm{Zn}$ and carbon disulfide ${ }^{(79)}$, and dimethyl sulfur(81) and diethyl sulfur(83) for that of $S$, are also used in MOCVD ZnS. The instability of DMZ and the pre-reaction of DMZ with $\mathrm{H}_{2} \mathrm{~S}$ have pushed researchers to try other MO sources such as those listed above.

The introduction of luminescent centers like $\mathrm{Mn}$ into MOCVD prepared $\mathrm{ZnS}$ film can be done using either thermal diffusion or the addition of dopant gases during deposition (in situ doping). A thin $\mathrm{Mn}$ layer, as a diffusion source, can be evaporated either before or after the deposition of $\mathrm{ZnS}$. Good results for the thermal diffusion of Mn into $\mathrm{ZnS}$ have been obtained by post deposition annealing at temperature higher than $400{ }^{\circ} \mathrm{C}$. On the other hand, lower annealing temperature is preferred for thermal diffusion in order not to damage the transparent conductor film. A compromise can be made 
by annealing at $400^{\circ} \mathrm{C}$ for one hour.(84)(85) The diffusivity of $\mathrm{Mn}$ in $\mathrm{ZnS}$ at $400^{\circ} \mathrm{C}$ was found to be $1.4 \times 10^{-15} \mathrm{~cm}^{2} / \mathrm{sec}^{(41)}$

TCM, tricarbonyl methylocyclopentadienyl manganese, was first introduced to in-situ dope $\mathrm{ZnS}$ with $\mathrm{Mn}$ by Wright. ${ }^{(80)}$ Its chemical structure is shown in Fig. 8(a). The concentration of TCM in the gas flowing into the reactor can be controlled by simply adjusting the - temperature of the TCM container. Good EL performance of in-situ doped $\mathrm{ZnS}$ has been reported.(44)(84)(86) The problem with the use of TCM is that a substrate temperature above $500^{\circ} \mathrm{C}$ is needed for

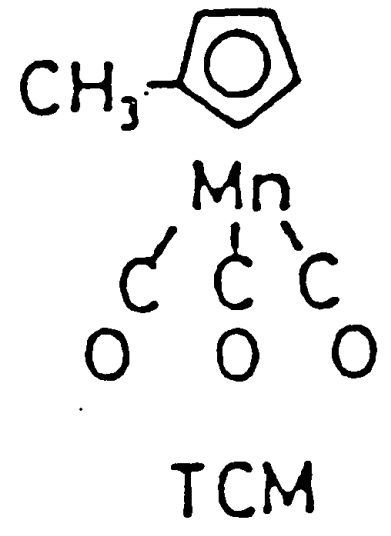

(a)

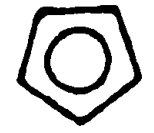

$\mathrm{Mn}$<smiles>c1ccccc1</smiles>

CPM

(b)

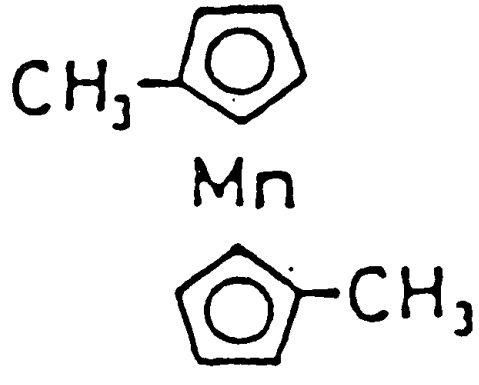

BCPM

Fig. 8. The chemical structure of (a) TCM, (b) CPM, and (c) BCPM. ${ }^{(82)}$ 
efficiently decomposing TCM into Mn for doping ZnS, which causes poor crystallinity of the $\mathrm{ZnS}$ film and damage of ITO due to the reaction of $\mathrm{ZnS}$ with ITO. Decomposing TCM into $\mathrm{Mn}$ before introducing TCM to the reaction chamber by photo-irradiation has been shown to be possible at a reduced temperature of $400{ }^{\circ} \mathrm{C}$ (83). However, it is still much higher than the optimum growth temperature, around 280 to $350^{\circ} \mathrm{C}$, of MOCVD ZnS.(78)(81) $\mathrm{CPM}^{(82)}$, di$\pi$-cyclopentadienyl manganese, and $B C P M(82)$, bimethylcyclopentadienyl manganese, have also been used as doping sources. Chemical structures of CPM and BCPM are shown in Fig. 8(b) and (c). Both of them can be completely decomposed at the optimum temperature of $\mathrm{ZnS}$ depositions. Colored EL devices prepared using MOCVD are made possible by introducing different dopants into ZnS. However, thermal diffusion is the only way available to dope those luminescent centers other than $\mathrm{Mn}$, due to the lack of stable metalorganic compounds for rare earth elements. ${ }^{(82)}$ The performance of these phosphors is listed in Table 1.

\subsection{Insulating Layers}

Because of the environment of continuously high operating voltage, most ac TFEL devices use the MISIM structure to concentrate most of the electric field across the phosphor and to minimize electrical breakdown of the devices. In the MISIM structure, the phosphor layer is sandwiched between two insulating 
layers. The whole device can be viewed as three capacitors connected in series. The memory effect in ac TFEL devices is mainly due to the charge stored in the insulating layers. The origin of electrons for impact excitation of luminescent centers is believed to be at the interface of the phosphor and the insulating layers. Therefore, a suitable choice of materials for insulating layers not only can reduce the risk of electrical breakdown but also will influence the efficiency, brightness, stability and power needed of the devices.

The first purpose of using insulating layers is to protect the device from electrical breakdown with minimum voltage drop. This can be done by choosing an insulator with high dielectric capacitance as shown in Eq. 8 in section 2. A suitable insulating layer in ac TFEL devices should be able to retain the charge stored at high field. The maximum charge capacity $q_{\max }$, the density of charge stored just before breakdown, for an insulator can be calculated as

$$
\mathrm{q}_{\max }=œ \times \mathrm{E}_{\mathrm{BD}}
$$

where $œ$ is the dielectric permittivity and $E_{B D}$ is the electric field strength at breakdown. Because the behavior of the phosphor layer and the insulating layers in ac TFEL devices can be seen as three capacitors in series, in order to generate EL without breakdown, $q_{\max }$ of the insulating layers must be larger than the charge density of the phosphor layer when operated at voltage over the threshold 
for EL. It was found that $q_{\max }$ of the insulating layers should be at least three times that of the phosphor layer. ${ }^{(87)}$ A value of 3 $\mu \mathrm{C} / \mathrm{cm}^{2}$ is often required for the charge-storage capacity of the insulating layers. ${ }^{(46)}$ This requirement can be achieved by choosing a material with either a high dielectric strength or a high breakdown field (See Table 3).

In addition to high charge capacity, other common requirements for dielectric materials must be considered when choosing suitable insulating films. Those are: no leakage current under low applied voltage, self-healing dielectric breakdown, low pin-hole-defect density, high resistivity, good adhesion to $\mathrm{ZnS}$, and suitable chemical stability (insensitivity to moisture and oxygen) throughout the processes of device fabrication. Candidates and physical properties of usually used insulating materials are listed in Table 3.

The choice of dielectric materials can be roughly separated into two types: amorphous oxides and nitrides like $\mathrm{Al}_{2} \mathrm{O}_{3}$ and $\mathrm{Si}_{3} \mathrm{~N}_{4}$, and ferroelectric insulators like $\mathrm{BaTiO}_{3}$ and $\mathrm{PbTiO}_{3}$. Evaporated $\mathrm{SiO}$ was widely used as insulating layer for EL devices in the 1960s because of the easier preparation process. ${ }^{(5)}$ E-beam evaporated $\mathrm{Y}_{2} \mathrm{O}_{3}$ was first introduced for making long-life ac TFEL devices by Inoguchi(3) and was frequently used in the 1970s. However, $\mathrm{Y}_{2} \mathrm{O}_{3}$ was found to have reproducibility problems. $\mathrm{Si}_{3} \mathrm{~N}_{4}$ has a problem of adhesion and unwanted film stress which causes peeling. $\mathrm{Al}_{2} \mathrm{O}_{3}$ has a processing problem due to the degradation of ITO and deficiency of oxygen.(46) $\mathrm{BaTiO}_{3}$ performs well in amorphous form but tends to crystallize and 


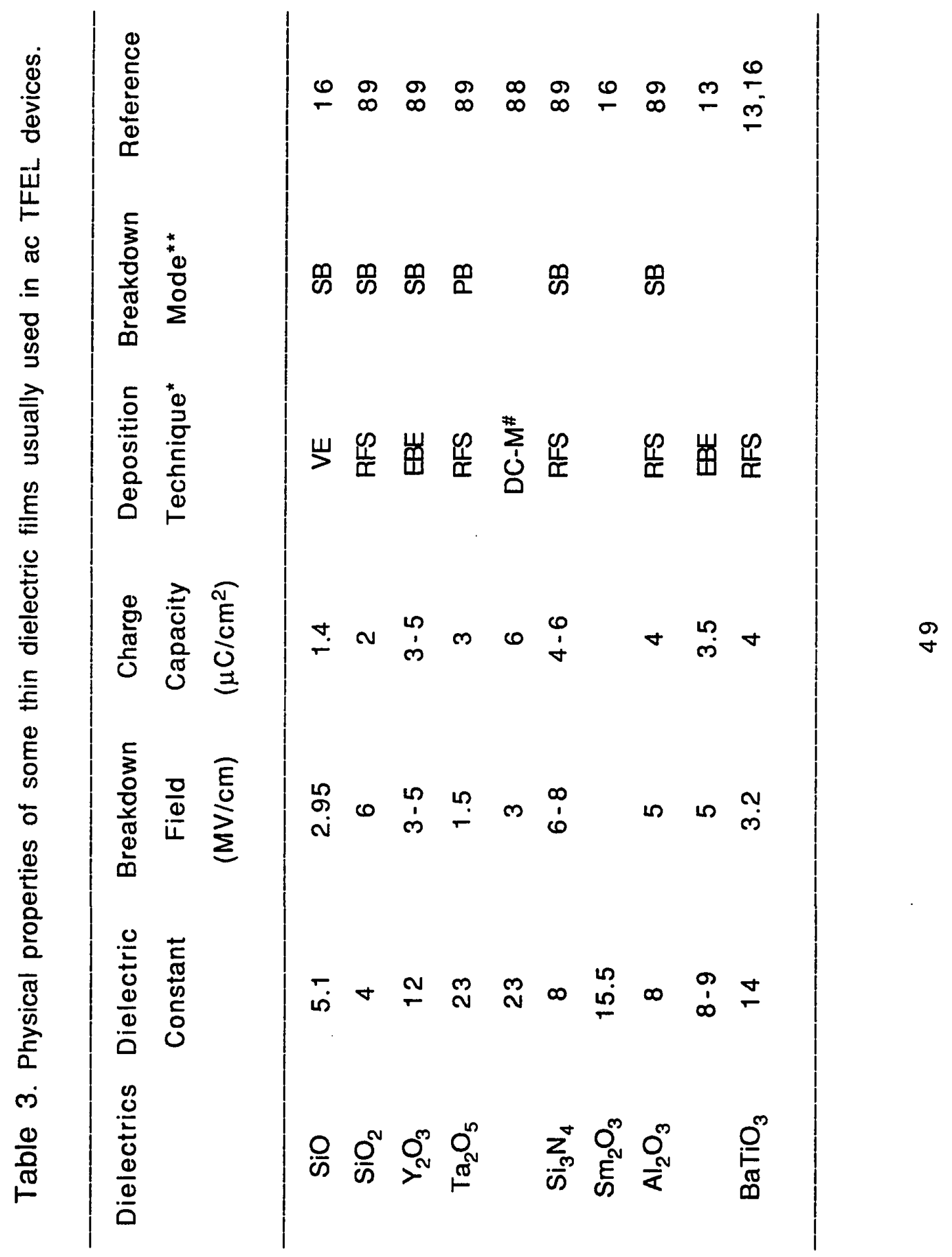




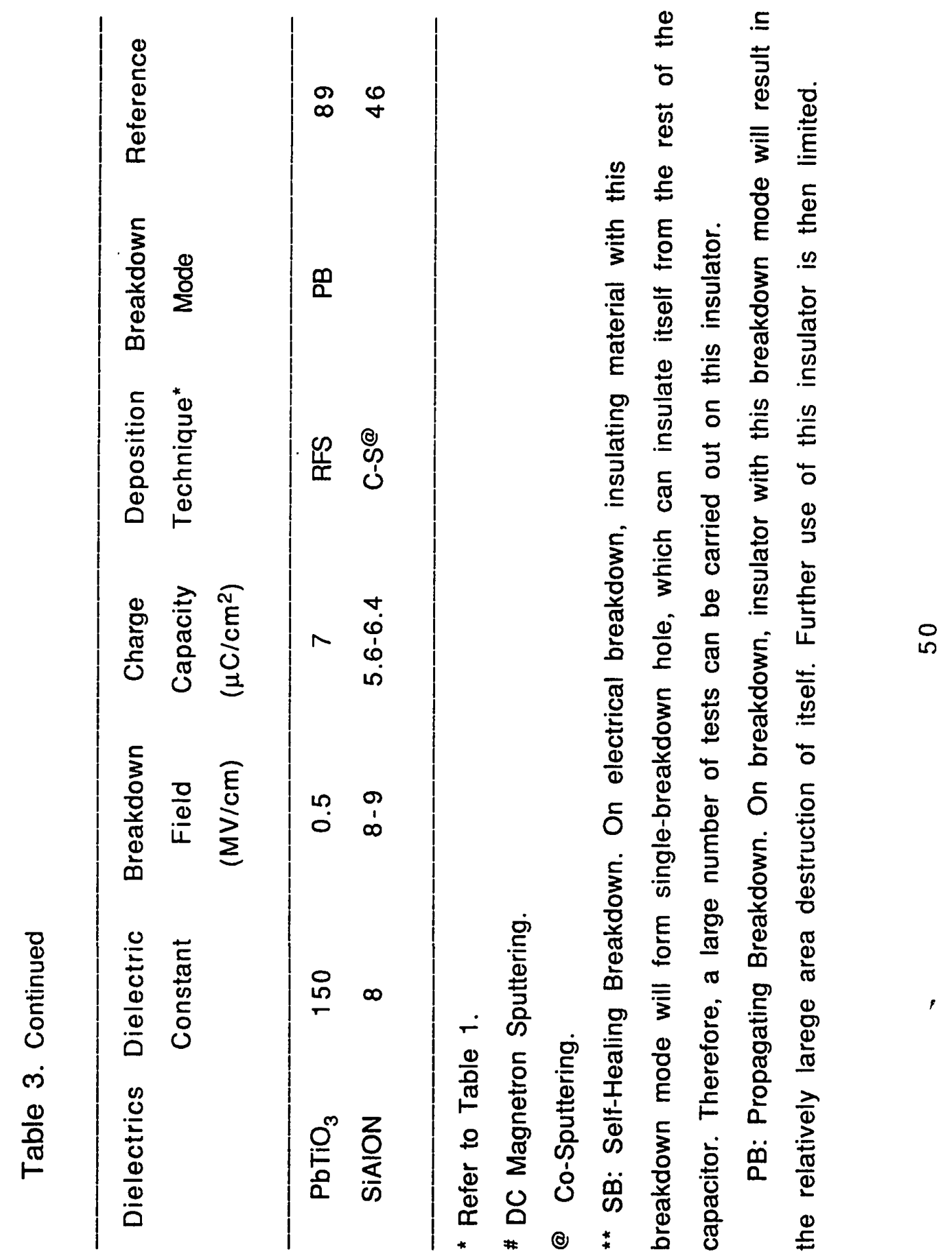


loses dielectric strength during annealing. (46) $\mathrm{Ta}_{2} \mathrm{O}_{5}$ is an excellent dielectric material but will react with $\mathrm{ZnS}: \mathrm{Mn}$ during annealing. Its poor adhesion to ITO is also a problem. ${ }^{(90)}$ Because of the difficulties of finding an ideal single material for insulating films, further studies are now being focused on finding a suitable combination of two or more of those dielectric materials which partially fit requirements. Those studies can be briefly classified into two approaches: a composite dielectric, and a stacked dielectric. SIAION is one of the candidates for the former.(46) The stacking of $\mathrm{Ta}_{2} \mathrm{O}_{5}$ with $\mathrm{Al}_{2} \mathrm{O}_{3}{ }^{(46)(88)}$ or $\mathrm{SiO}_{2}{ }^{(90)(91)}$ is the latter.

Finally, it should be noted that, although double-insulatinglayer-type ac TFEL devices have many advantages over LCDs and plasma displays, the need for a high voltage to drive the devices makes EL displays less attractive than LCDs in applications like notebook computers, where a low power supply is critical. In order to lower the voltage required to drive EL displays, using thinner insulating films with high dielectric strength becomes a major approach. 


\subsection{Transparent Conductor and Glass Substrate}

In ac TFEL devices, two different types of electrodes, transparent conductor and metal, are used to introduce power to the devices. The requirements for a transparent conductor are simply that it must be as transparent as a glass and as nearly conductive as a metal as possible. However, to reach this goal is never an easy job. The only way to obtain such a transparent conductor is to create electron degeneracy in a wide band gap oxide by controlling the introduction of proper doping. ${ }^{(92)} \mathrm{Tin}$ oxide, $\mathrm{SnO}_{2}$, is the first transparent-conductor material to be commercialized. Other oxides like indium oxide, zinc oxide and cadmium stannate are also candidates suitable for the transparent conductor. Among them, tin doped indium oxide, usually called indium-tin-oxide (ITO), is found to be easier to be patterned.(93)(94) Therefore, ITO is preferred for use as a transparent conductor in opto-electronic devices, where photolithography techniques are required to make small pixels for displays. ITO film, whose transparency is greater than $85 \%$ at 540 $\mathrm{nm}$, is a degenerate n-type semiconductor with a resistivity around $10^{-2} \mathrm{ohm}-\mathrm{cm}$ and a band gap of $3.75 \mathrm{eV} .^{(95)}$ The conduction mechanism in ITO films involves the defect energy states associated with oxygen vacancies. ${ }^{(96)}$

Vacuum evaporated aluminum (Al) films are usually used as a back electrode. The addition of reflected $E L$ from the $A l$ back electrode can increase the brightness and efficiency of $E L$ 
generation, but, in turn, causes the contrast loss of devices. In addition, ambient light is also a killer of the contrast. The use of a black layer and filter, or polarizer, can minimize this contrast loss. ${ }^{(97)}$

Like LCDs and plasma displays, ac TFEL devices are very sensitive to contamination by alkali ions like sodium. Therefore, a low-sodium-content glass is preferred for the substrate.

\subsection{Summary}

To create an on-the-wall full-color display is the driving force for keeping the $R$ \& $D$ on ac TFEL displays going. The principal structure of $E L$ devices consists of a phosphor layer which is sandwiched between two insulating layers, an evaporated aluminum film and a transparent-conductor layer. ITO, indium-tin-oxide, is usually used as the transparent conductor. The whole device is prepared on a low-sodium-content glass.

Although ZnS:Mn EL devices have been commercialized and the use of $\mathrm{ZnS}$ as a host to generate green and red EL has met with some success, the difficulties of using doped $\mathrm{ZnS}$ phosphor to generate bright blue and the need for high voltage to drive the devices are pushing researchers to examine other materials and process techniques. In order to have blue EL, the band gap of the host material must be larger than $3.3 \mathrm{eV}$. Cerium doped $\mathrm{SrS}$ is thought to be the best of all the candidates for blue luminance. To lower the 
driving voltage, an appropriate thin dielectric with charge-storage capacity of $3 \mu \mathrm{C} / \mathrm{cm}^{2}$ or higher is needed for the insulating layers. Dielectrics which meet this criteria are usually either those with a high dielectric constant like $\mathrm{Ta}_{2} \mathrm{O}_{5}$ or those with a high breakdown field like $\mathrm{BaTiO}_{3}$. However, almost none of the known dielectrics fits in well with the requirements. The use of composite dielectrics or stacked dielectrics may solve the problem of finding a proper insulator.

The choice of dopant will affect not only the color of the generated light but also the efficiency and brightness of EL devices. An ideal dopant should have large impact cross section and be free from non-radiative energy-transfer channels. Rare earth elements, which can offer a wide variety of emission in the range of visible wavelength, were once thought to be a good choice. However, from a viewpoint of brightness, of all the rare earths, only terbium ( $\mathrm{ZnS}: \mathrm{Tb}$ ) is found to be fit for practical applications. Because of the difficulty of finding a bright blue phosphor for full color EL displays, people try to employ different fabrication techniques to compensate for the nature of poor brightness of blue EL. Among those techniques, the patterned phosphor structure and the patterned filter method seem to be most attractive.

ZnS can be prepared using five different methods. They are evaporation, if sputtering, atomic layer epitaxy (ALE), MOCVD and spray prolysis. Although vacuum evaporation and E-beam evaporation are the major methods used in the industry now, they will be 
gradually replaced by other methods which meet the stringent requirements for the manufacture of large-area EL displays. Sputtering is poor for the deposition of large-area thin films. ALE prepared phosphor films perform well but cost too much. MOCVD and spray techniques, which offer good crystallinity, stoichiometry and film uniformity for $\mathrm{ZnS}$, seem to be the better choice than any others for ac TFEL fabrication. 


\section{Experimental Procedures}

Several ac TFEL devices with the MISIM structure as shown in Fig. 1 were made in this study. ZnS:Mn was used as the phosphor layer. $\mathrm{Sm}_{2} \mathrm{O}_{3}$ and $\mathrm{Al}_{2} \mathrm{O}_{3}$ were tested as insulating layers, but were both found in preliminary experiments to exhibit poor adhesion to both ZnS and ITO films. Consequently, SiO was chosen for the insulating layers due to the ease of preparation and good adhesion to ITO and ZnS. All devices were fabricated on an ITO-coated glass substrate. Silver films were deposited as top electrodes to contact the devices. The details of these device-fabrication procedures are as follows.

\subsection{Device Fabrication}

One-inch-square glass sheet coated with a $120 \mathrm{~nm}$ ITO film, purchased from Precision Engineered Glass Products (5570 Harbor St., Commerce, CA 90040), and called IRR Pyrex, was used as the substrate. Although the glass was purported to have been cleaned by the manufacturer, the side of the glass coated with ITO film was cleaned using pure acetone solvent at least three times before using. The resistance of the ITO films, before deposition, was measured to be 50 ohms using the two-point-probe method with a distance of 1.5 $\mathrm{cm}$ between probes. This is consistent with a bulk resistivity of 


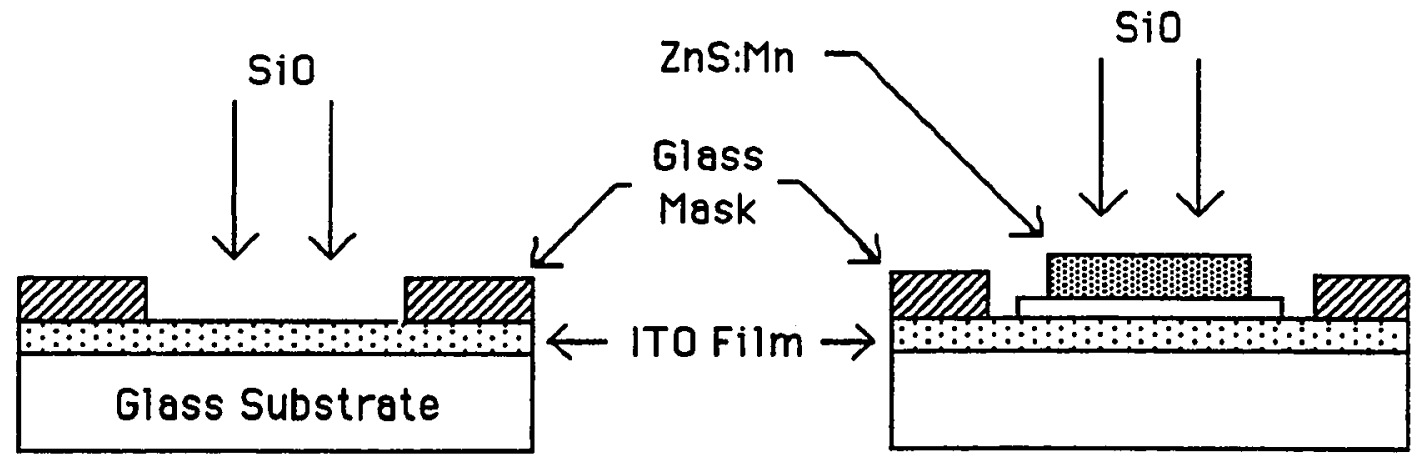

(a)

(d)

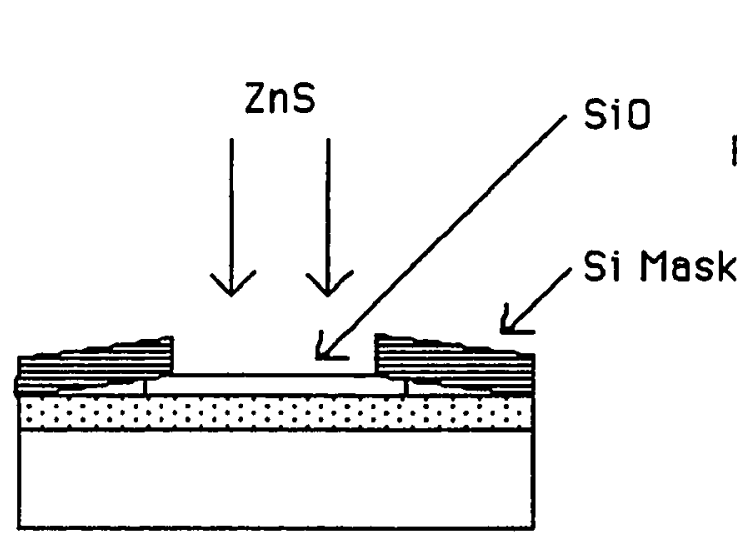

(b)

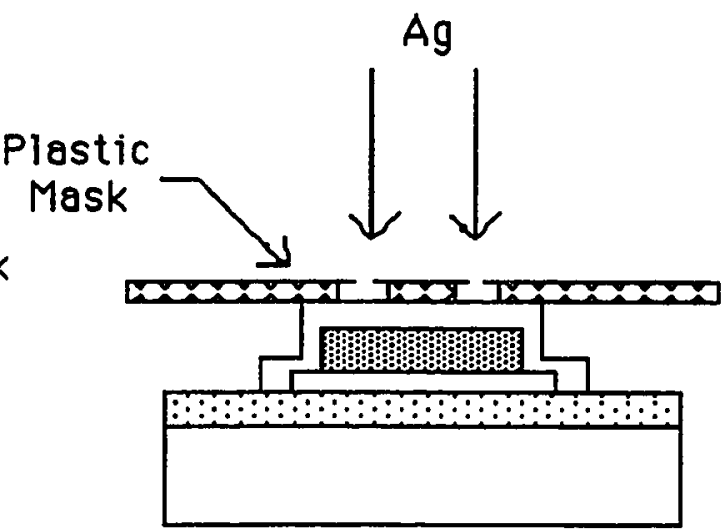

(e)

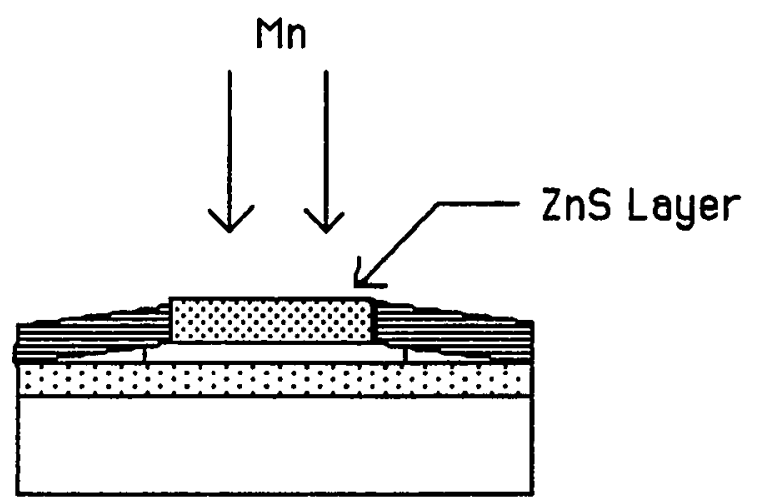

(c)

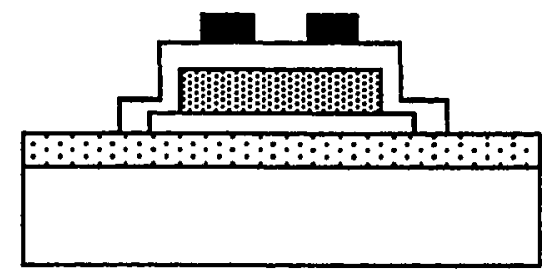

(f)

Fig. 9. Process sequence of fabricating ac TFEL devices. 
about $10^{-3}$ ohm-cm.

The process sequence for fabricating an ac TFEL device is shown in Fig. 9. A thin SiO film was the first layer to be deposited onto the ITO glass using vacuum evaporation. In order to deposit the SiO layer selectively, a shaped and cleaned cover glass with a thickness less than $0.2 \mathrm{~mm}$ was used as a mask as shown in Fig. 9(a). A measured quantity of $\mathrm{SiO}$ was put into a molybdenum boat and the evaporation was carried out under a pressure less than $10^{-3}$ torr. The average deposition rate of SiO was about $350 \mathrm{~A} / \mathrm{min}$. The SiO was deposited as strips with dimensions of $5 \mathrm{~mm} \times 10 \mathrm{~mm}$ and a thickness of 0.2 to $0.5 \mu \mathrm{m}$, typically.

The phosphor layer ZnS:Mn was prepared using additive-typeMOCVD ZnS and thermally diffused Mn. In this work, Mn was chosen to be introduced after deposition of the $\mathrm{ZnS}$. The MOCVD reactor used was built by Marc Jensen(98) and modified by Hoa Do(99). The schematic diagram of the geometry of the MOCVD reactor used is shown in Fig. 10. Unlike the conventional way of using a bubbler, $1 \%$ dimethyl zinc (DMZ) and 10\% hydrogen sulphide $\left(\mathrm{H}_{2} \mathrm{~S}\right)$ in nitrogen were used as reactants to deposit $\mathrm{ZnS}$ in the MOCVD chamber. The basic reaction of $D M Z$ with $\mathrm{H}_{2} \mathrm{~S}$ is given by

$$
\left(\mathrm{CH}_{3}\right)_{2} \mathrm{Zn}_{(\mathrm{g})}+\mathrm{H}_{2} \mathrm{~S}_{(\mathrm{g})} \cdots \mathrm{ZnS}_{(\mathrm{s})}+2 \mathrm{CH}_{4(\mathrm{~g})}
$$

The SiO-evaporated glass substrate was put on a substrate holder and heated by two quartz lamps beneath. The flow rates of DMZ and 


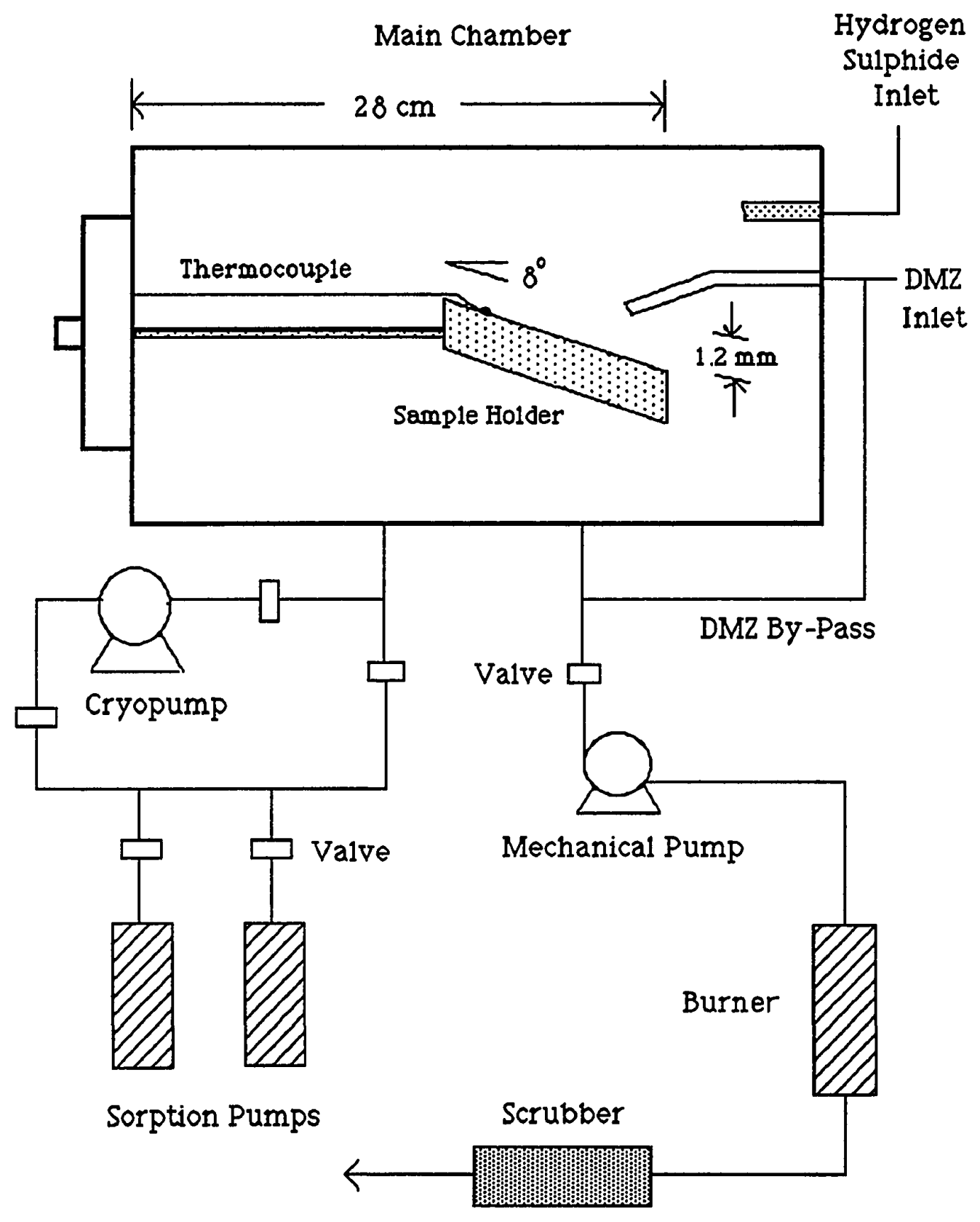

Fig. 10. Schematic illustration of MOCVD reactor used in this work. 
$\mathrm{H}_{2} \mathrm{~S}$ were controlled at the optimum conditions proposed by Kashani(100), which were $15 \mathrm{sccm}$ for $\mathrm{H}_{2} \mathrm{~S}$ and $22 \mathrm{sccm}$ for DMZ. Deposition was carried out under a pressure of 1 torr and a temperature of $300{ }^{\circ} \mathrm{C}$. This vacuum was achieved by using the mechanical pump and adjusted by means of the inline valve (Fig. 10). In order to minimize mobile-ion introduction into the ITO substrate at this high temperature, patterned and undoped Si strips were used as a mask as shown in Fig. 9(b). The thickness of the deposited ZnS was controlled by adjusting the time of reaction of $D M Z$ with $\mathrm{H}_{2} S$. Before starting the reaction, DMZ was pumped through the by-pass pipe. However, the uniformity of the deposited ZnS was very poor due to the geometry of the reactor. The deposition rate and thickness distribution of deposited $\mathrm{ZnS}$ relative to the positions on the substrate holder are listed in Appendix A. After the ZnS deposition, nitrogen gas was introduced to bring the pressure of reaction chamber back to atmosphere.

The introduction of dopant $\mathrm{Mn}$ into $\mathrm{ZnS}$ was achieved using thermal diffusion. This process can be separated into two steps: pre-deposition of $\mathrm{Mn}$ on $\mathrm{ZnS}$ and drive-in. Pure manganese (99.99\%) was used as the source. Mn was deposited using vacuum evaporation under a pressure of less than $10^{-3}$ torr as shown in Fig. 9(c). The distance between the tantalum boat and the substrate was adjusted to be $18 \mathrm{~cm}$. Therefore, the amount of dopant to be diffused into $\mathrm{ZnS}$ can be estimated using the small-area-source equation(101) 


$$
d M_{r} / d A_{r}=M_{e} /\left(\pi \times h^{2}\right)
$$

where $M_{r}$ and $M_{\theta}$ are the mass of deposit on substrate at evaporation height $h$ and the total mass of evaporated material, respectively. $A_{r}$ is the area of deposit. More calculations related to Eq. 15 and the way to estimate dopant content in $\mathrm{ZnS}$ are present in Appendix B.

After the evaporation of $\mathrm{Mn}$, the substrate was quickly shifted to a vacuum chamber, where drive-in would be held, in order to minimize the possible oxidation of the $\mathrm{Mn}$ film. The chamber used was the same as that for depositing ZnS. Substrate heating did not begin until the pressure in the vacuum chamber was reduced to less than $1 \times 10^{-6}$ torr using sorption pumps and a cryopump. Thermal diffusion was carried out at $400{ }^{\circ} \mathrm{C}$ for one hour. The time to heat the substrate holder to $400^{\circ} \mathrm{C}$ from room temperature was about 26 minutes. After thermal diffusion, the device was left in the vacuum chamber for natural cooling. The resistance of ITO films after this thermal cycle was measured to be from 46 to 48 ohms using the same method as mentioned earlier (v.s. 50 ohms for the as-reacted ITO).

The second thin SiO layer was subsequently deposited on the $\mathrm{ZnS}: \mathrm{Mn}$ layer using the same vacuum-evaporation method as that of the first SiO layer (Fig. 9(d)). The thickness of the second insulating layer was controlled to be about the same as that of the first. Finally, silver films around 20 to $50 \mathrm{~nm}$ in thickness were selectively superimposed on the second SiO layer using vacuum 
evaporation to pattern contacts (Fig. $9(e)$ ). The ac TFEL device with MISIM structure as shown in Fig. 9(f) was then completed and ready for testing and characterization. The thickness of each layer was measured after each deposition using an Alpha-step profiler. Measurements are reported in section 5.

\subsection{Characterization Methods}

The characterization of the ac TFEL devices was carried out using the set-up as shown in Fig. 11. The sinusoidal voltage used to drive the ac TFEL devices was first generated from a function generator with desired frequency and then amplified by a transformer. This power supply was connected to the ITO and silver films of the device. Both the applied voltage and current were measured by means of digital meters. EL, which was generated after applied voltage reached threshold, was detected by a photomultiplier tube (Philips, type XP2102B). During the measurements, both the photomultiplier tube and the EL device were placed in a light-tight container to avoid the influence from ambient light. The distance between the photomultiplier and the EL device was around $11 \mathrm{~cm}$. The signal of the luminance measured was separated into two channels; one of them being sent to a Tektronix 455 oscilloscope and the other being sent to an HP $X-Y$ recorder as the $Y$ axis input. The signal of input voltage was also sent to the oscilloscope and to the $X-Y$ recorder as the $X$ axis input. Therefore, the phase 


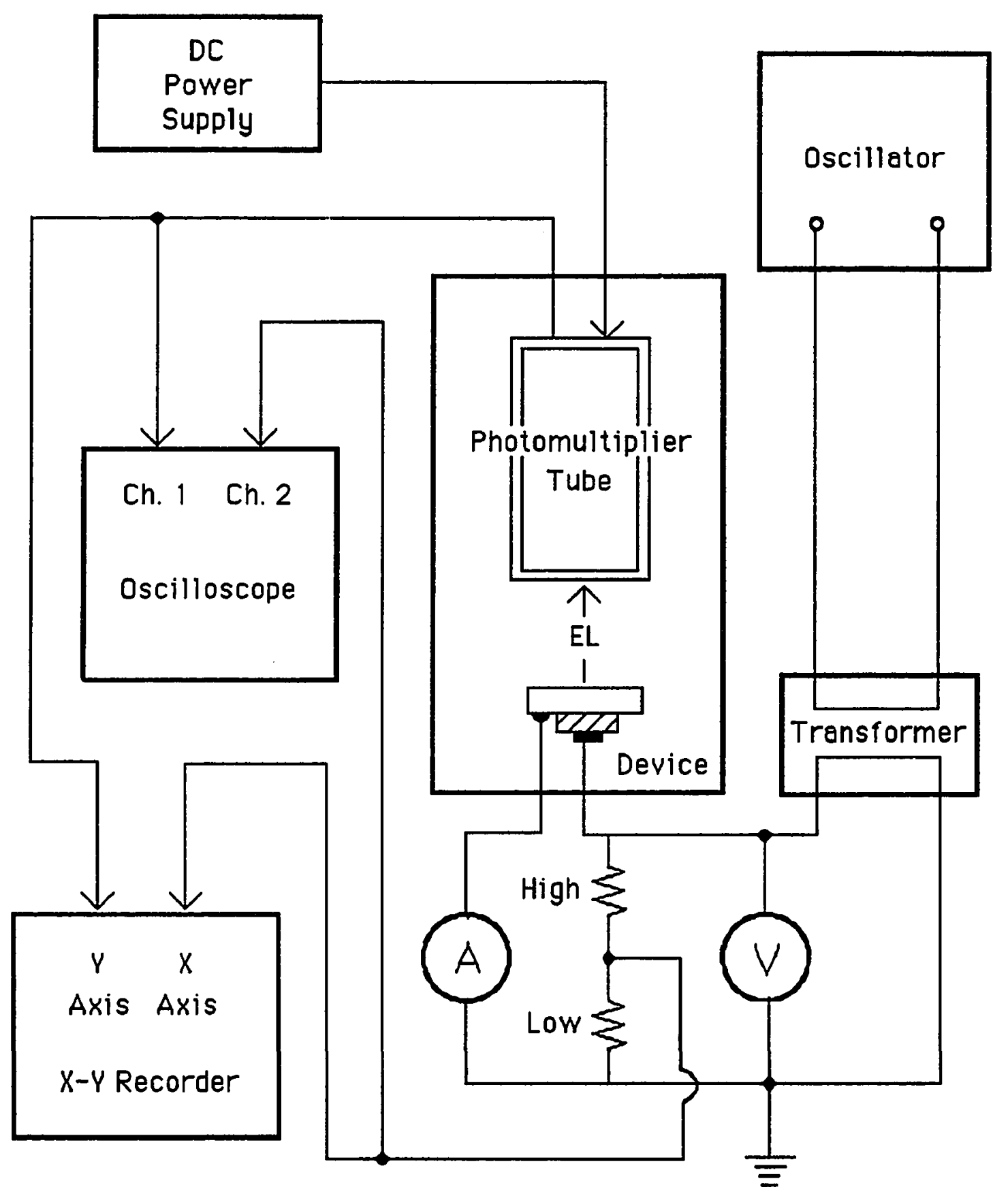

Fig. 11. The schematic diagram of experimental set-up for measuring and recording relative brightness, voltage and current. 
relationship between applied voltage and generated $\mathrm{EL}$, and the data of relative brightness of luminance versus frequency, current and voltage applied to the device could be gathered and studied. All measurements were performed at a frequency of $1 \mathrm{kHz}$, which is typical for the operation of EL devices

Attempts were also made to measure the emission spectrum of the EL using a Perkin-Elmer LS-3 Fluorescence spectrophotometer. However, no reliable results could be achieved in this work due to the insensitivity of the spectrophotometer and the low luminance intensity of the EL obtained from these sample devices. 


\section{Experimental Results and Discussion}

$\mathrm{ZnS}: \mathrm{Mn}$ films were prepared using the fabrication procedures described in the previous section. Previously, the crystallinity of MOCVD ZnS films had been observed to be mainly (111) by Kashani using X-ray diffraction. $\left.{ }^{100}\right)$ Serious peeling between the SiO layer and the ITO film was observed when the time of thermal diffusion was too long ( greater than 4 hours), or when total thickness of the combined first SiO layer and $\mathrm{ZnS}$ layer was too thick (over 3

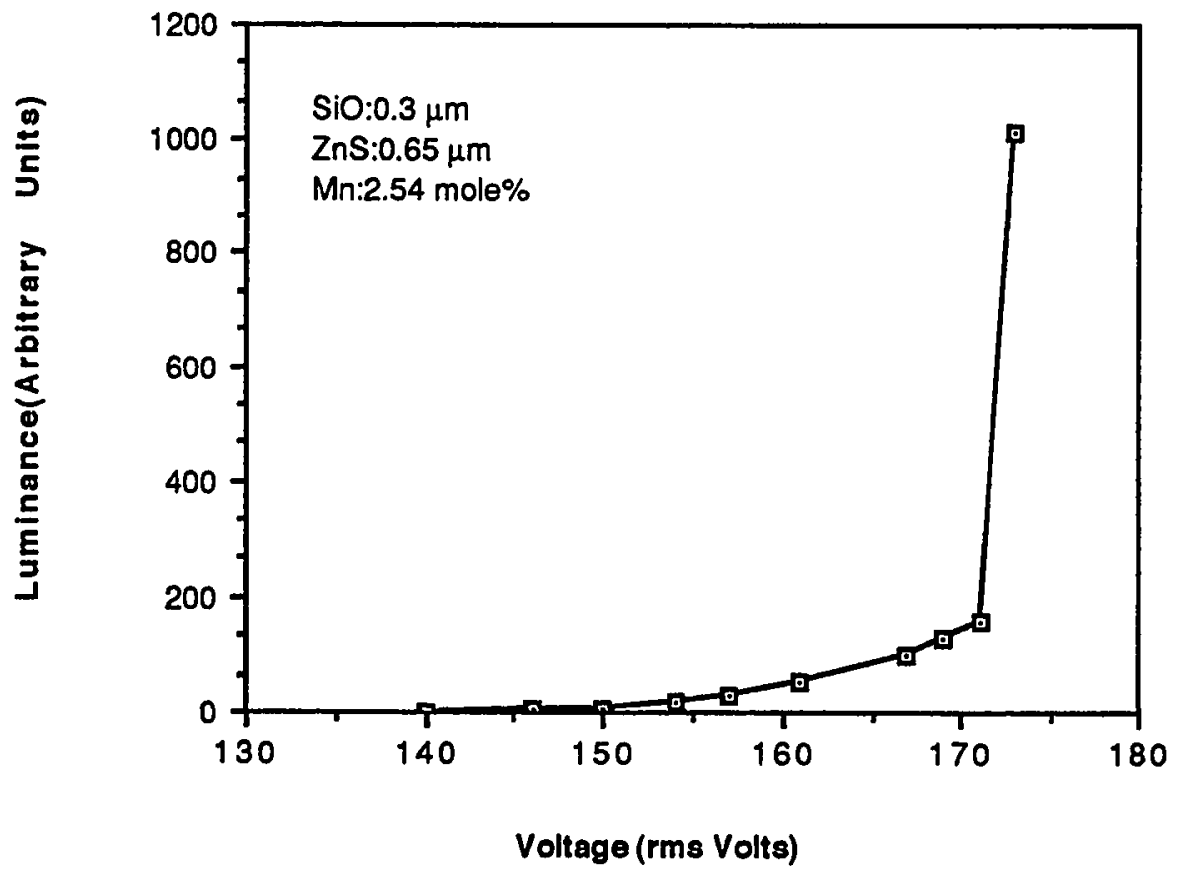

Fig. 12. The typical luminance-voltage characteristics of MOCVD prepared $\mathrm{ZnS}: \mathrm{Mn}$ ac TFEL devices under $1 \mathrm{k} \mathrm{Hz}$ sinusoidal excitation. (Data gathered from sample \#5, device 1-1.) 


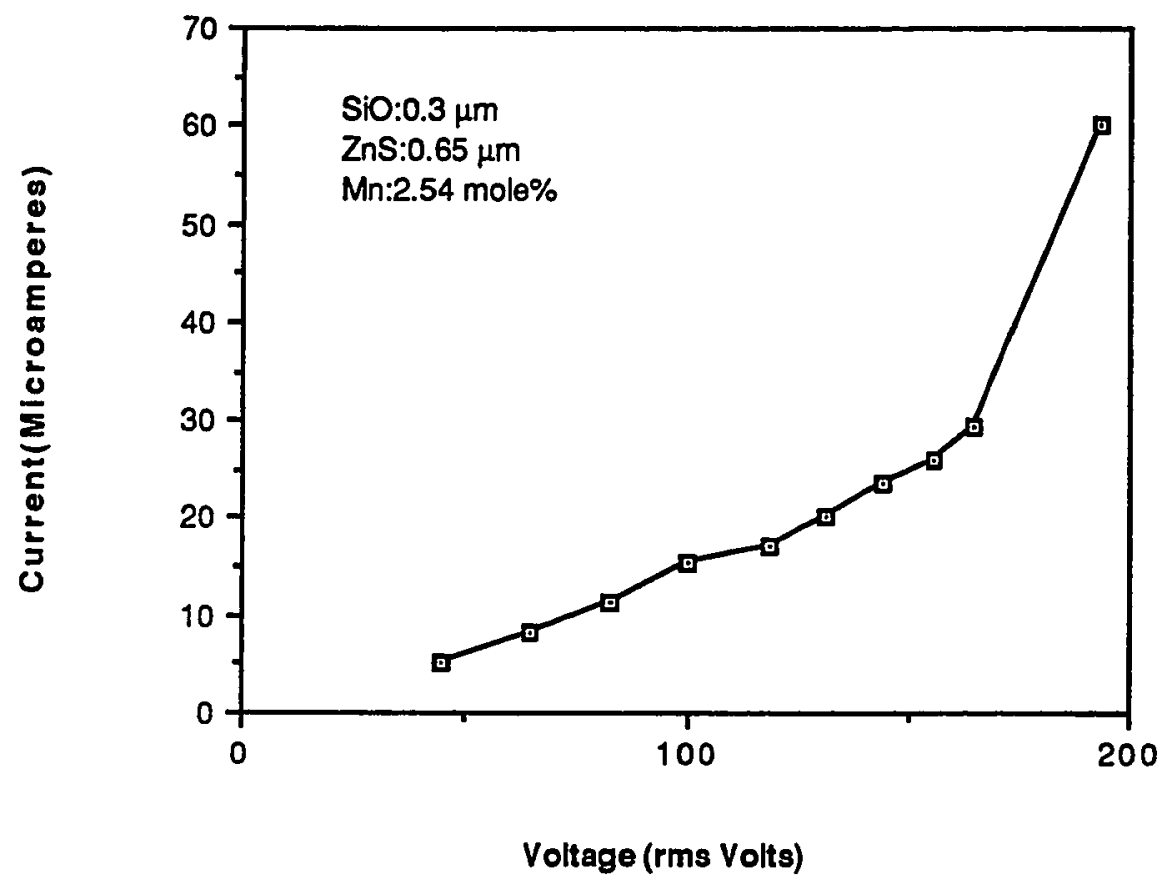

Fig. 13. The current-voltage characteristic of ac TFEL devices under $1 \mathrm{k} \mathrm{Hz}$ sinusoidal power. Note that an rms voltage of 100 volts corresponds to peak-to-peak excursions of +140 volts to -140 volts. (Data gathered from sample \#5, device 1-1.)

microns). Low field breakdown was frequently observed, presumably due to the introduction of impurities.

The typical luminance-voltage(L-V) and current-voltage(I-V) characteristics of the ac TFEL devices are shown in Fig. 12 and Fig. 13, respectively. The intensity of the generated EL increases dramatically as applied voltage exceeds 172 volts in this case. The 


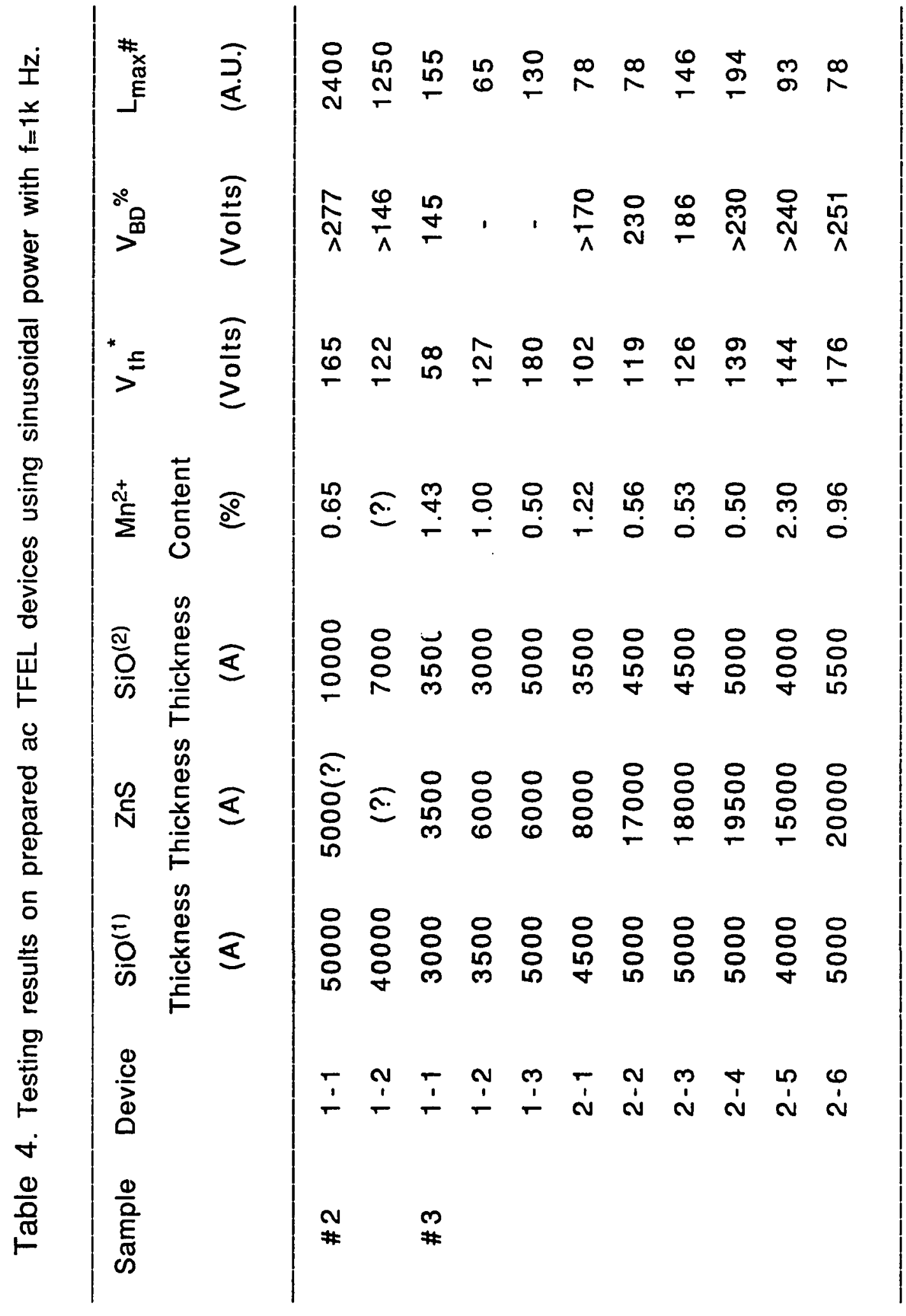

$N$ 


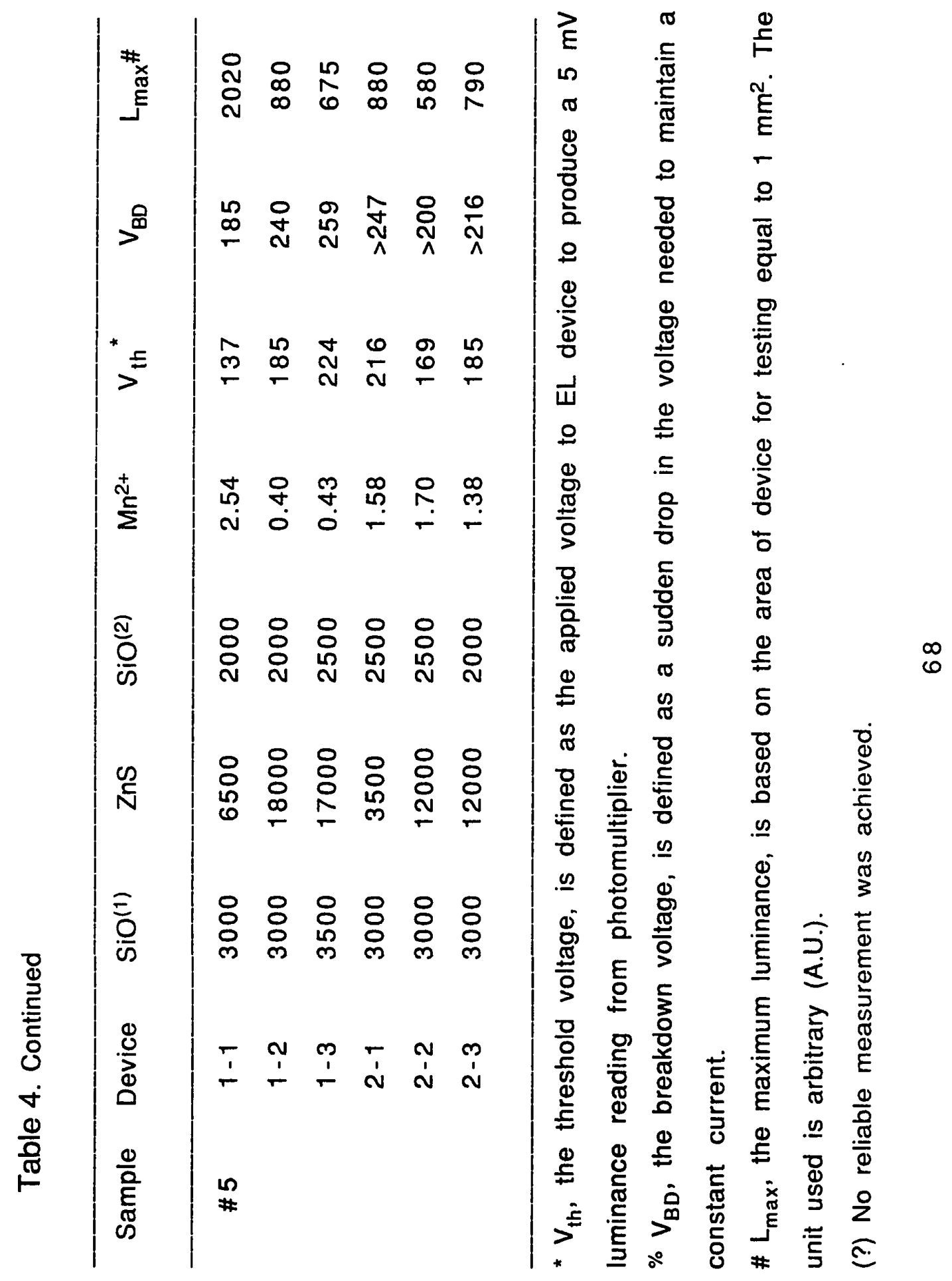




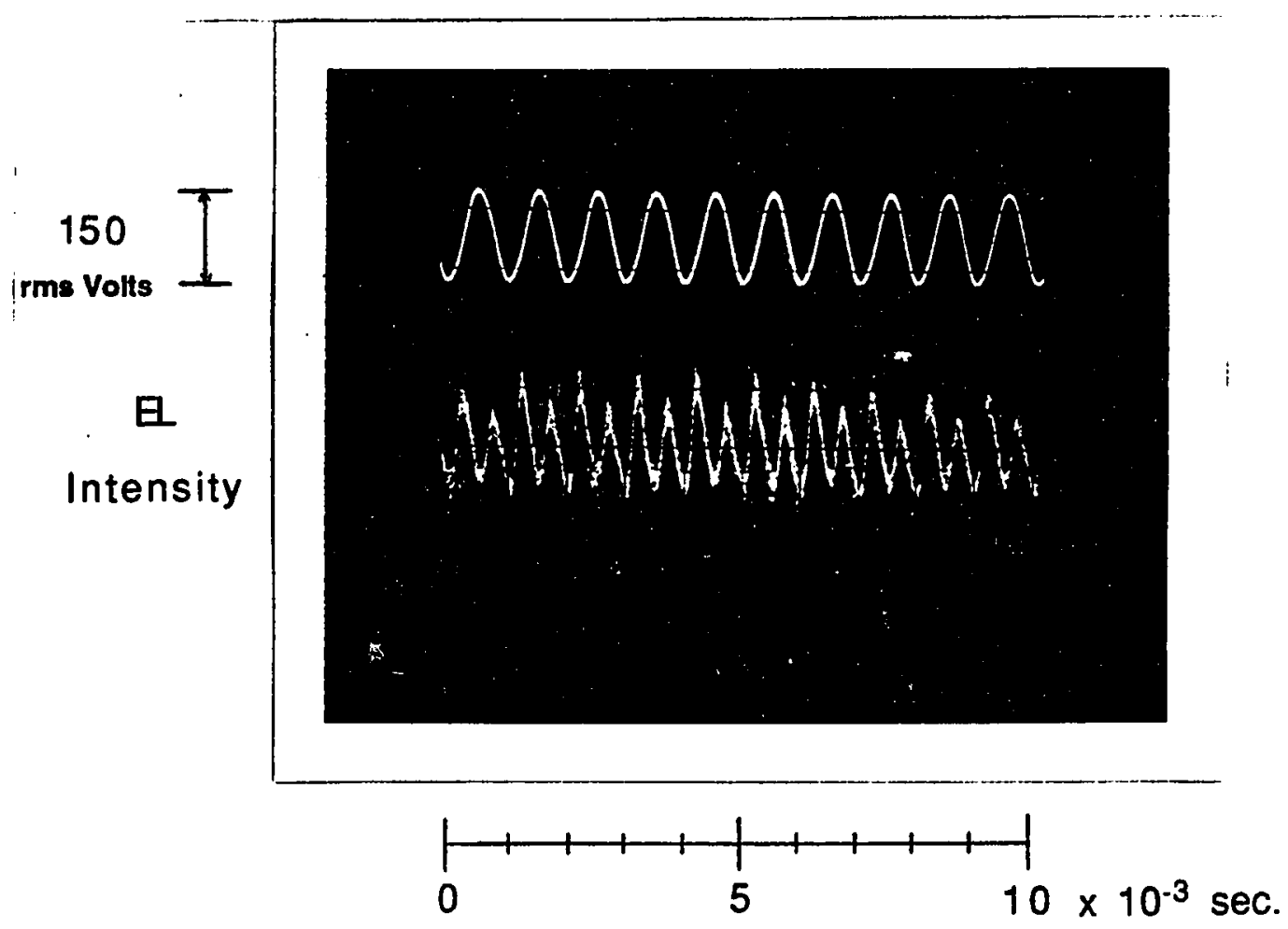

Fig. 14 The emission waveforms of ac TFEL devices(lower) under $1 \mathrm{k}$ $\mathrm{Hz}$ sinusoidal voltage excitation(upper).

slope of I-V curve also increases abruptly as voltage is measured over 172 volts. The order of current is a few tens of microamperes. The breakdown voltage of this device is 185 volts. Although the intensity of EL was expected to saturate at high voltages, this phenomenon was not observed in any of the present studies. This may be due to the limitation of the power supply used which could not be fine controlled, especially at applied voltages over threshold where the ac TFEL devices become extremely sensitive to the power applied. This disadvantage also caused most of the tested devices to undergo electrical breakdown. Table 4 lists the experimental results 
of the threshold voltage and breakdown voltage of the tested samples and the thickness of each layer. The common threshold voltage observed is between 100 and $220 \mathrm{rms}$ volts. The breakdown voltage is between 170 and 260 volts.

Fig. 14 shows the phase relationship between the applied voltage and the emission waveform of ac TFEL devices under a sinusoidal voltage excitation with frequency $1 \mathrm{~K} \mathrm{~Hz}$. It was obvious that, in each cycle of the applied voltage pulse, the generated EL was observed with two peaks, one brighter than the other. Each peak occurred when the electric field in the device attained a maximum value. The brighter EL output was generated when the metal electrode was negative, and the ITO electrode was positive (see setup in Fig. 11). Fig. 15 shows the relative intensity of these two light outputs with respect to the applied voltage. The observed threshold voltage was 165 volts. Separation of the emission waveform was clearly observed as the applied voltage reached 194 volts. In Fig. 15, the observed luminance intensity of the brighter peak is always higher than that of the lighter peak during the increase of the applied voltage to the device. The reason why the intensity of these two peaks is not the same is thought to be due to the poor distribution of $\mathrm{Mn}$ in $\mathrm{ZnS}$ and/or the asymmetry of the devices. In this study, there was no secondary light output(102) observed in each EL peak when the applied voltage approached zero. 


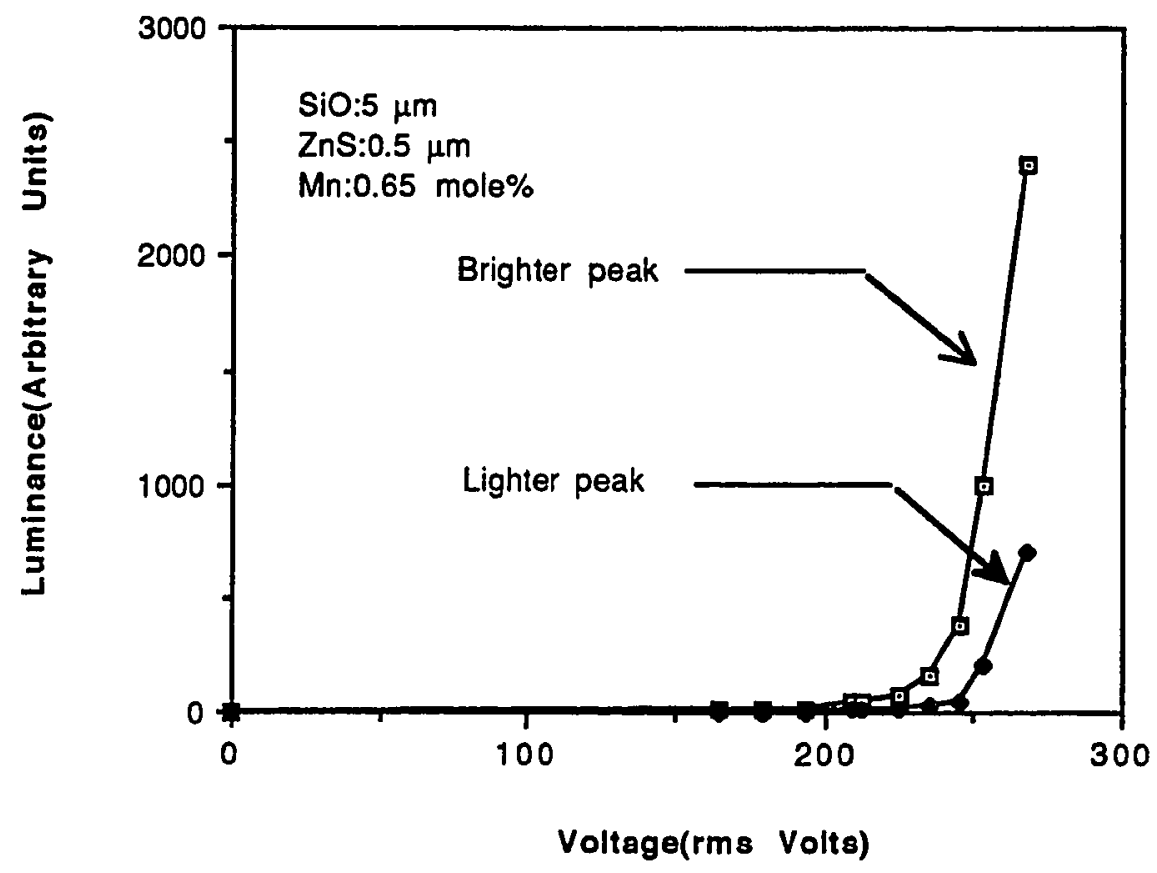

Fig. 15. The relative intensity between the brighter EL peak and the lighter under $1 \mathrm{k} \mathrm{Hz}$ sinusoidal voltage excitation. (Data gathered from sample \#2, device 1-1.)

In this work, more than six samples of ac TFEL devices were fabricated. However, only three of them generated luminance sufficient to be studied and the results are listed in Table 4. Furthermore, the behavior of the tested devices was extremely different from one to another in spite of the similarity of dopant content and thickness of the layers. For example in sample 5, although the $\mathrm{Mn}$ content and thicknesses of $\mathrm{SiO}$ and phosphor layers of device 1-2 are almost identical to those of device 1-3, the 
measured luminance intensity is very different. As can be seen from Table 4, the intensity of luminance of devices from sample 3 is on the average ten times less than that of devices from samples 2 and 5. These frustrating results indicate the poor reproducibility of ac TFEL devices using this fabrication procedure. This might be due to the introduction of EL-killer impurities like $\mathrm{H}_{2} \mathrm{O}$, mobile ions and oxygen into the $\mathrm{SiO}$ and $\mathrm{ZnS}$ layers during device fabrication. Therefore, although the thicknesses of SiO and $\mathrm{ZnS}$ and the $\mathrm{Mn}$ contents in this work were set up to vary from 0.2 to $0.5 \mu \mathrm{m}, 0.35$ to $2 \mu \mathrm{m}$ and 0.4 to $2.54 \%$, respectively, no reliable conclusions as to the influence of thickness and Mn content on luminance intensity could be drawn. The results obtained for various thickness and dopant levels are listed in Table 4.

The previously discussed memory effect of ac TFEL devices was observed in the present study and is shown in Fig. 16. A sinusoidal voltage with frequency fixed at $1 \mathrm{k} \mathrm{Hz}$ was applied to the device, and the rms voltage was gradually increased from zero to the value near breakdown, which was about 220 rms volts in this case. This gathered luminescence v.s. voltage curve as the applied voltage increases is called the forward path in this study. This path is similar to that in Fig. 12. If, under fixed frequency, we started to decrease the applied rms voltage from $220 \mathrm{rms}$ volts toward zero, the L-V characteristic during this voltage decrease would differ from that of the forward path. This new path of L-V characteristic is called the reverse path in this work. This phenomenon is known as 
the memory effect. The reason why this effect occurs is believed to be due to the storage of positive charge in the insulating layers during power application, which distorts the conduction band of $\mathrm{ZnS}$ and enhances the field at the cathode interface(34)(35). The different charge storage between the phosphor layer and the insulating layer can be inferred from the I-V curve in Fig. 13. Details of this effect were discussed in section 2. The largest voltage difference between the forward and the reverse paths in Fig. 16 is around 100 volts.

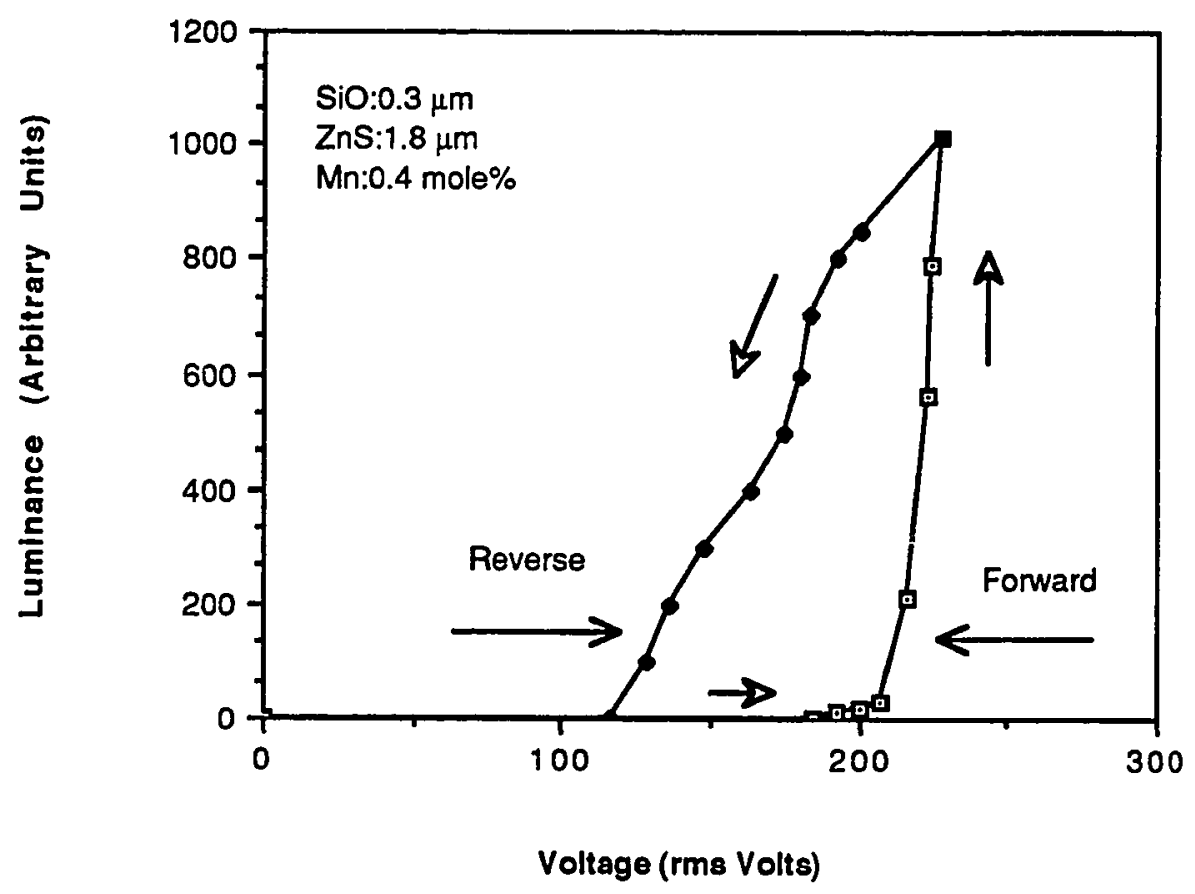

Fig. 16. The typical hysteretic behavior of the luminance-voltage characteristic of ac TFEL devices at frequency $1 \mathrm{kHz}$. (From sample \#5, device 1-2.) 


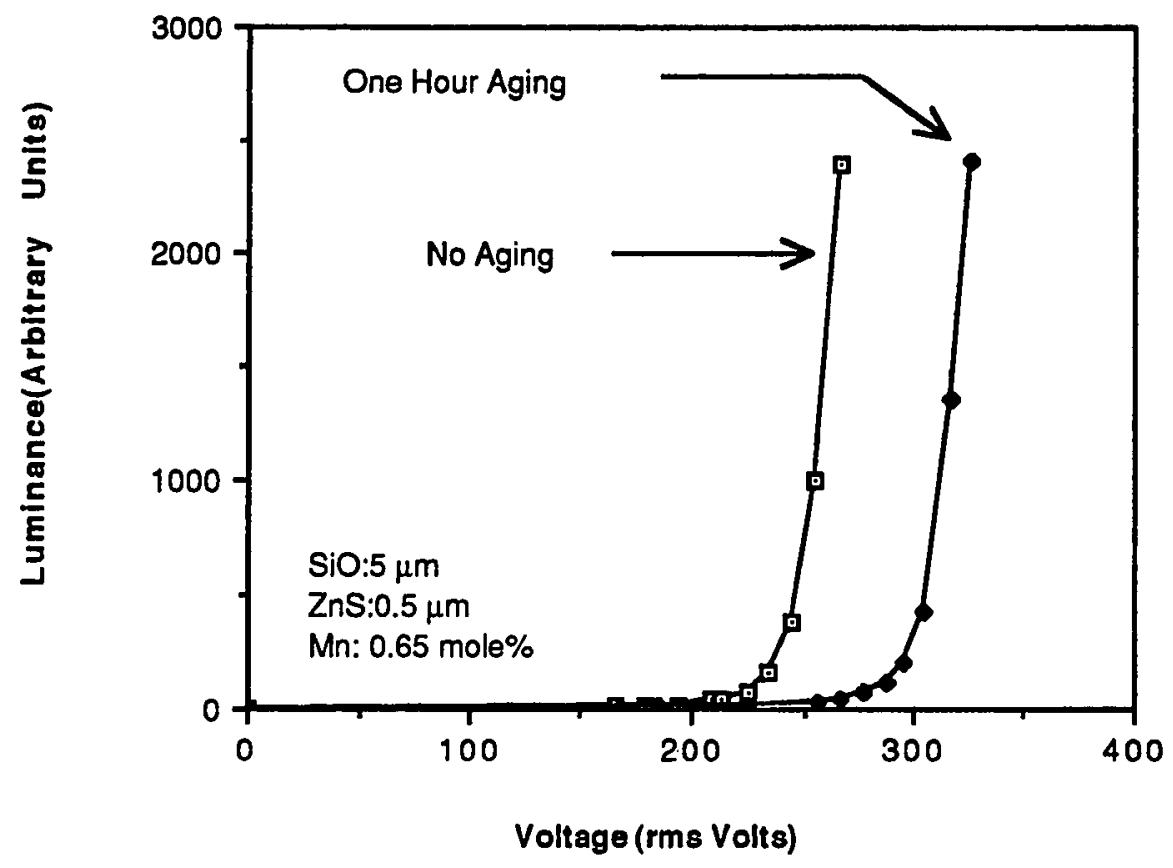

Fig. 17. The change of luminance-voltage characteristic of ac TFEL devices with respect to the time of power applied at frequency 1 $\mathrm{kHz}$. (From sample \#2, device 1-1.)

From Fig. 12 to 16 we know that luminance intensity is a function of the voltage applied to the devices. In addition, ac TFEL devices' behavior is also known to be time-dependent. Fig. 17 shows two $L-V$ curves. The curve in the left was gathered from a new device during the initial application of power. The curve in the right was gathered from the same device after being continuously applied a voltage of 160 to $170 \mathrm{rms}$ volts at $1 \mathrm{kHz}$ for one hour. As can be 
seen, the threshold voltage and the voltage required for the same level of maximum brightness of this device increased about 50 volts from their original values after being applied for one hour. This phenomenon of change in threshold voltage and the L-V curve with respect to the time of operation of this device is called "aging." Aging effects in ac TFEL devices are thought to be due to the generation of deep traps in bulk $\mathrm{ZnS}$ and the interface between $\mathrm{ZnS}$ and the insulating layers, which result in a higher threshold voltage being required to initiate electron tunneling.(103) Therefore, the voltage needed to create EL increases with increasing time of operation of the devices.

Although some researchers have suggested that ac TFEL devices have to be aged for at least 20 hours before becoming stable enough for study(52), the aging history of the EL devices in this work was far less than that. Another problem encountered in this work is that, although the devices were powered to about 2/3 to 1/2 of breakdown field during aging, the generated EL starts to fade after one to two hours of aging. In other words, the active area where EL occurred tended to decrease with increasing aging time. The longest aging time, or the lifetime, of the EL devices was about six hours in this work. After six hours, the area where EL occurred was reduced to less than $1 / 5$ of the original contact area. The average lifetime of the present ac TFEL devices was about two to three hours. This short lifetime might be due to one or more of the mechanisms of device degradation proposed by other researchers. $(104)-(107)$ These 
degradation or failure modes can be roughly classified into three types: dielectric breakdown(104)(105)(106), accumulation of sodium ions near the phosphor-insulator interface ${ }^{(105)}$, and the delamination of deposited films under continuous high fields, especially the metal film(104)(106). Delamination of metal films caused by the electrostatic force was thought to be the main reason. Further work is suggested to studying the degradation modes of ac TFEL devices.

Although ZnS:Mn phosphor can be made using MOCVD, and the fabrication process used in this work can successfully make ac TFEL devices, the reproducibility and lifetime of the resulting devices are so poor that accurate performance data were difficult to collect. Better process control of the deposition of $\mathrm{ZnS}: \mathrm{Mn}$ and the insulating layers may be of help in minimizing the introduction of impurities and thus promoting the brightness and the reproducibility of EL devices. In addition, replacing SiO with other materials having a higher charge-storage capacity, together with better process control, may prolong the lifetime of prepared ac TFEL devices. 


\section{Conclusion}

The first problem this study met was the poor reproducibility in performance of the ac TFEL devices. This was probably due to the introduction of impurities into the deposited layers during device fabrication, when a clean room facility was not available for this work. However, some fabricated devices generated good intensity of luminance so that the basic studies of their performance could be achieved.

Electroluminescence was generated as the applied voltage exceeded a threshold value, which was in the range of 100 to 220 rms volts. The emission waveform of the generated luminance clearly separated into two peaks of differing brightness at an applied voltage approximately 30 volts higher than that of the threshold. Each peak occurred when the electric field in the device attained a maximum value. Low field breakdown of the devices was frequently observed, presumably due to the introduction of impurities. The normal breakdown voltage ranged from 170 to 260 rms volts in this study.

The previously reported memory effect of ac TFEL devices was also observed in this study. The voltage difference between the increasing voltage and decreasing voltage paths was around 100 volts. Aging studies and lifetime testing of prepared ac TFEL devices were carried out at a voltage $2 / 3$ to $1 / 2$ of the normal breakdown 
voltage. The average lifetime of the prepared ac TFEL devices was found to be about two to three hours. The area within each device where EL occurred tended to decrease as the aging proceeded. This short lifetime of the prepared ac TFEL devices is presumed to be due to the degradation of SiO layers and contact electrodes under a continuous application of high field.

The experimental procedures used in this work were found to be capable of successfully fabricating ac TFEL devices. However, no reliable conclusions of the influence of the thickness of $\mathrm{ZnS}$ and SiO layers and of $M n$ content on luminance intensity could be drawn due to the poor reproducibility and short lifetime of the prepared devices. Replacing SiO with other insulator materials having a high melting point and good charge capacitance, along with a better control of the deposition of each layer during device fabrication, are thought to be capable of overcoming the problems encountered in this work. 


\section{References}

1. Gail M. Robinsion, "Display Systems Leap Forward," Design News, Vol. 45, No. 3, (1989) p. 53.

2. Gene Smarte and Nicholas M. Baran, "Face to Face," BYTE, Vol. 13, No. 9, (1988) p. 243.

3. T. Inoguchi, M. Takeda, Y. Kakihara, Y. Nakata and M. Yoshida, "Stable High-Brightness Thin-Film Electroluminescent Panels," Society of Information Display, International Symposium: Digest of Technical Papers, Vol. 5, (1974) p. 84.

4. G. Destriau, "Research into the Scintillations of Zinc Sulfides to Alpha Rays," Journal de Chimie Physique et de Physico-Chimie Blologiques, Vol. 33, (1936) p. 587.

5. M. J. Russ and D. I. Kennedy, "The Effects of Double Insulating layers on the Electroluminescence of Evaporated ZnS:Mn Films," Journal of Electrochemical Society: Solid State Science, Vol. 14, No. 11, (1967) p. 1066.

6. J. Ohwaki, H. Kozawaguchi and B. Tsujiyama, "Effect of Electric Field and Polarity on Light Emission in Metal-InsulatorSemiconductor Structure Thin-Film Electroluminescent Devices," Japanese Journal of Applied Physics, Vol. 22, No. 1, (1983) p. 65.

7. J. Ohwaki, H. Kozawaguchi and B. Tsujiyama, "Green Electroluminescence in Low-Voltage-Driven Metal-Insulator- 
Semiconductor Structure Devices," Japanese Journal of Applied Physics, Vol. 22, No. 7, (1983) p. 1133.

8. G. O. Muller and R. Mach, "Physics of Electroluminescence Devices," Journal of Luminescence, Vol. 40/41, (1988) p. 92.

9. D. C. Krupka, "Hot-Electron Impact Excitation of $\mathrm{Tb}^{3+}$ Luminescence in ZnS:Tb ${ }^{3+}$ Thin Films," Journal of Applied Physics, Vol. 43, No. 2 (1972) p. 476.

10. P. M. Alt, "Thin-Film Electroluminescent Displays: Device Characteristics and Performance," Proceedings of the Society of Information Display, Vol. 25, No. 2 (1984) p. 123.

11. D. H. Smith, "Modeling ac Thin-Film EL Devices," Leurnal of Luminescence, Vol. 23, Nos. 1/2, (1981) p. 209.

12. K. Okamoto and S. Miura, "Excitation Mechanism in Thin-Film Electroluminescent Devices," Applied Physics Letters, Vol. 49, No. 23 (1986) p. 1596.

13. R. Mach and G. O. Muller, "Review Article," Physica Status Solidi A, Vol. 69, No. 1 (1982) p.11.

14. E. Bringuier, "Charge Transfer in ZnS-Type Electroluminescence," Journal of Applied Physics, Vol. 66, No. 3 (1972) p. 1314.

15. H. Yoshiyama, S. H. Sohn, S. Tanaka, and H. Kobayashi, "Excitation Mechanism Based on Field-Induced Delocalization of Luminescent Centers in CaS:Eu ${ }^{2+}$ and SrS:Ce ${ }^{3+}$ Thin-Film Electroluminescent Devices," Springer Proceedings in Physics: Electroluminescence, Vol. 38 (1989) p. 48. 
16. Lawrence E. Tannas Jr., Flat-Panel Displays and CRTs: Electroluminescence, Van Nostrand Reinhold Company Inc., (1985) Chapter 8.

17. G. Vincent, A. Chantre, and D. Bois, "Electric Field Effect on the Thermal Emission of Traps in Semiconductor Junctions," Journal of Applied Physics, Vol. 50, No. 8 (1979) p. 5484.

18. S. M. Sze, Physics of Semiconductor Devices: Tunneling Diodes, John Wiley \& Sons, (1981) Chapter 9.

19. K. A. Neyts and P. D. Visschere, "Analytical Model for Thin-Film Electroluminescent Devices," Journal of Applied Physics, Vol. 68, No. 8 (1990) p. 4163.

20. J. W. Allen and S. G. Ayling, "On the Possibility of Obtaining HotElectron Distribution Functions from the Electroluminescence of Rare-Earth-Doped Crystals," Journal of Physics C: Solid State Physics, Vol. 19 , No. 18 (1986), p. L369.

21. F. J. Bryant, W. E. Hagston and A. Krier, "On the Hot-Electron Energy Distribution in the Electroluminescence of Rare-Earth-Doped Zinc Chalcogenides," Journal of Physics C: Solid State Physics, Vol. 19, No. 18 (1986) p. L375.

22. Y. Jiaqi, S. Yongrong, X. Xumou, L. Baozhu, and Z. Guozhu, "The Impact Cross Section of Electroluminescence Centers," Springer Proceedings in Physics: Electroluminescence, Vol. 38 (1989) p. 24.

23. S. Mengyan and X. Xurong, "Impact Excitation Cross Scection in Electroluminescence," Springer Proceedings in Physics: Electroluminescence, Vol. 38 (1989) p. 32. 
24. D. Hommel, H. Hartmann, F. J. Bryant, M. J. R. Swift, W. Busse, and H.-E. Gumlich, "On the Stability of Rare Earth Centers in ||$-\mathrm{VI}$ Compounds" Springer Proceedings in Physics: Electroluminescence, Vol. 38 (1989) p. 101.

25. J. Benoit, P. Benalloul, A. Geoffroy, and C. Barthou, "Decay of the Yellow Emission of $\mathrm{Mn}^{2+}$ in $\mathrm{AC}$ Thin Film Electroluminescent Devices," Physica Status Solidi A, Vol. 105, No. 2 (1988) p. 637.

26. O. Goede, W. Heimbrodt, and D. D. Thong, "Non-Exponential ZnS:Mn Luminescence Decay due to Energy Transfer," Physica Status Solidi B, Vol. 126, No. 2 (1984) p. K159.

27. D. D. Thong, W. Heimbrodt, D. Hommel, and D. Goede, "Optical Study of ZnS:Mn Thin Films with High Mn Concentration," Physica Status Solidi A, Vol. 81, No. 2 (1984) p. 695.

28. R. Tornqvist, "Manganese Concentration Dependent Saturation in ZnS:Mn Thin Film Electroluminescent Devices," J.Applied Physics, Vol. 54 , No. 7 (1983) p. 4110.

29. T. Matsuoka, J. Kuwata, M. Nishikawa, Y. Fujita, T. Tohda, and A. Abe, "Influence of Oxygen and Metal Oxide Impurities in ZnS:Mn Films on Characteristics of ELectroluminescent Devices," Japanese J. of Applied Physics, Vol. 27, No. 8 (1988) p. 1426.

30. G. O. Muller, J. Neugebauer, R. Mach, and U. Reinsperger, "Excited State Interaction in the Dopant System in $\mathrm{ZnS}: \mathrm{Mn}$ Electroluminescence Devices," dournal of Crystal Growth, Vol. 86, Nos. $1-4$ (1988) p. 890. 
31. J. M. Langer, "Auger Effect in Semiconductors: Why does it matter for Electroluminescence?" Springer Proceedings in Physics: Electroluminescence, Vol. 38 (1989) p. 16.

32. J. W. Allen, "Impact Excitation and Auger Quenching of Luminescent Centers in Crystals, with Special Reference to ZnS:Mn," Journal of Physics C: Solid State Physics, Vol. 19, No. 31 (1986), p. 6287.

33. T. Inoguchi and S. Mito, Topics in Applied Physics: Electroluminescence, Springer-Verlag Berlin Heidelberg, Vol. 17, (1977) p. 197.

34. W. E. Howard, O. Sahni, and P. M. Alt, "A Simple Model for the Hysteretic Behavior of ZnS:Mn Thin Film Electroluminescent devices," J. Applied Physics, Vol. 53, No. 1 (1982) p. 639.

35. K. C. Yang, S. John T. Owen, "Mechanisms of the NegativeResistance Characteristics in AC Thin-Film Electroluminescent Devices," IEEE Transaction on Electron Devices, Vol. ED-30, No. 5 (1983) p. 452.

36. S. Holland, I. C. Chen, T. P. Ma, and C. Hu, "On Physical Models for Gate Oxide Breakdown," IEEE Electron Device Letters. Vol. EDL-5, No. 8 (1984) p. 302.

37. H. E. Boesch, Jr. and F. B. Mclean, "Interface State Generation Associated with Hole Transport in Metal-Oxide-Semiconductor Structure," J.Applied Physics, Vol. 60, No. 1 (1986) p. 448.

38. Z. A. Weinberg and T. N. Nguyen, "The Relation between Positive Charge and Breakdown in Metal-Oxide Silicon Structure," J.Applied Physics, Vol. 61, No. 5 (1987) p.1947. 
39. H. Onnagawa, M. Shibata and K. Miyashita, "Current and Field Characteristics and Memory Mechanism of TFEL Devices," Japanese Journal of Applied Physics, Vol. 25, No. 1 (1986) p. 12.

40. E. Bringuier, "Electron Multiplication in ZnS-type Electroluminescent Devices," J.Applied Physics, Vol. 67, No. 11 (1990) p. 7040 .

41. J. M. Hurd and C. N. King, "Physical and Electrical Characterization of Co-Deposited ZnS:Mn Electroluminescent. Thin Film Structures," Journal of Electronic Materials, Vol. 8, No. 6 (1979) p. 879.

42. R. Mach and G. O. Muller, "Ballistic Transport and EL in IIB-VI and IIA-VI compounds," J. of Crystal Growth, Vol. 101, Nos. 1-4 (1990) p. 967.

43. Tom Manuel, "A Full-Color EL Display is Demonstrated by Planar," Electronics, Vol. 61, No. 11 (1988) p. 73.

44. K. Hirabayashi and H. Kozawaguchi, "Memory Effect in AC Thin Film ZnS:Mn Electroluminescent Devices Prepared by Metal Organic Chemical Vapor Deposition," Japanese J. of Applied Physics, Vol. 25, No. 5 (1986) p. L379.

45. R. O. Tornqvist, J. Anrson, J. Skarp, and V. P. Tanninen, "How the ZnS:Mn Layer Thickness Contributes to the Performance of AC ThinFilm Devices Grown by Atomic Layer Epitaxy (ALE)," IEEE Transaction on Electron Devices, Vol. ED-30, No. 5 (1983) p. 463. 
46. S. K. Tiku and G. C. Smith, "Choice of Dielectrics for TFEL Displays," IEEE Transaction on Electron Devices. Vol. ED-31, No. 1 (1984) p. 105.

47. H. Kozawaguchi, B. Tsujiyama and K. Murase, "Thin-Film Electroluminescent Device Employing $\mathrm{Ta}_{2} \mathrm{O}_{5}$ RF Sputtered Insulating Film," Japanese J. of Applied Physics, Vol. 21, No. 7 (1982) p. 1028.

48. S. I. Ohta, T. Tadokoro, S. Satou, and N. Yokoyama, "Low-VoltageDriven AC Thin Film EL Cell Containing A ZnS:Mn Film Deposited by Molecular Beam Epitaxy," Thin Solid Films, Vol. 162 (1988) p. 73.

49. T. Nire, T. Watanabe, N. Tsurumaki, A. Miyakoshi, and S. Tanada, "Multi-Source Deposition Method for ZnS and SrS Thin-Film Electroluminescent Devices," Springer Proceedings in Physics: Electroluminescence, Vol. 38 (1989) p. 218.

50. Y. Shimoptori, M. Yokoyama, K. Masugata, and K. Yatsui, "AC ThinFilm ZnS:Mn Electroluminescent Device Prepared by Intense Pulsed Ion Beam Evaporation," Springer Proceedings in Physics: Electroluminescence, Vol. 38 (1989) p. 232.

51. K. Hirabayashi, H. Kozawaguchi and B. Tsujiyama, "Color Electroluminescent Devices Prepared by Metal Organic Chemical Vapor Deposition," Japanese J. of Applied Physics, Vol. 26, No. 9 (1987) p. 1472.

52. J. Ohwaka, B. Tsujiyama and H. Kozawaguchi, "Red Electroluminescence and Crystallinity of $\mathrm{ZnS}: \mathrm{SmF}_{3}$ Thin Films," Japanese J_of Applied Physics, Vol. 23, No. 6 (1984) p. 699. 
53. T. Tohda, Y. Fujita, T. Matsuoka, and A. Abe, "New Efficient Phosphor Material ZnS:Sm, P for Red Electroluminescent Devices," Applied Physics Letters, Vol. 48, No. 2 (1986) p. 95.

54. K. Ukamoto, T. Yoshimi, K. Nakamua, T. Kobayashi, S. Sato, and S. Miura, "ZnS:TbOF Thin-Film Green Electroluminescent Panels Fabricated by Two-Target Sputtering," Japanese J. of Applied Physics, Vol. 28 , No. 8 (1989) p. 1378.

55. R. Fukao, H. Fujikawa, M. Nakamura, Y. Hamakawa, and S. Ibuki, "High-Luminance ZnS:Sm, F Thin Film Eiectroluminescent Devices Using Ferroelectric $\mathrm{PbTiO}_{3}$ Thin-Film," Springer Proceedings in Physics: Electroluminescence, Vol. 38 (1989) p. 164.

56. S. Okamoto, E. Nakazawa, and Y. Tsuchiya, "White-Emitting ThinFilm Electroluminescence Devices with SrS Phosphor Doubly Activated with Rare-Earth lons," Japanese J. of Applied Physics, Vol. 29, No. 10 (1990) p. 1987.

57. K. I. Onisawa, M. Fuyama, K. Toguchi, K. Tamura, and Y. A. Ono, "Luminance Improvement of Blue-Green Emitting SrS:Ce EL Cell by Controlling Vacuum Condition with Sulfur Addition," $\underset{\perp}{\perp}$ of Electrochemical Society, Vol. 135, No. 1 (1988) p. 2631.

58. Y. Tamura, J. Ohwaki, H. Kozawaguchi, and B. Tsujiyama, "Bright Blue Electroluminescence in $\mathrm{SrS}: \mathrm{CeCl}_{3}$ Thin-Films," Japanese $\mathrm{J}$. of Applied Physics, Vol. 25, No. 2 (1986) p. L105.

59. K. Tanaka et al., "High Brightness Red Electroluminescence in CaS:Eu Thin Film," Applied Physics Letters, vol. 48, No. 25 (1986) p. 1730 . 
60. M. Ogawa, T. Shimouma, S. Nakada, and T. Yoshioka, "Electroluminescence of Devices with Thin-Film CaS:Cs ${ }^{3+}, \mathrm{Cl}^{-}$as the Active Luminescent Layer," Japanese J. of Applied Physics, Vol. 24, No. 2 (1985) p. 168.

61. X. Fan and J. Zhang, "The Dependence of Near Band Edge Electroand Photoluminescence on Purity of Starting Materials in ZnSe Crystals," Springer Proceedings in Physics: Electroluminescence, Vol. 38 (1989) p. 109.

62. Y. Tamura and H. Kozawaguchi, "Sulphur Defects and Deep Levels in SrS:Ce Thin Films," Springer Proceedings in Physics: Electroluminescence, Vol. 38 (1989) p. 183.

63. Z. Zhang, Z. Li, B. Mei, X. Jiang, P. Wu, and S. Xu, "The Relation of Thin Film Electroluminescence and Photoluminescence Excitation Spectra," Springer Proceedings in Physics: Electroluminescence, Vol. 38 (1989) p. 105.

64. H. Fujiyasu, N. Katayama, H. Yang, K. Ishino, A. Ishida, M. Kaneko, and T. Ohiwa, "CdS-ZnS Superlattice Electroluminescent Device Prepared by Hot Wall Epitaxy," Springer Proceedings in Physics: Electroluminescence, Vol. 38 (1989) p. 116.

65. G. Zhong, C. Li, L. Meng, and H. Song, "Green AC Electroluminescence in ZnS Thin Films Doped with $\mathrm{Tb}, \mathrm{Er}$ and Ho lons and Concentration Quenching Models," Springer Proceedings in Physics: Electroluminescence, Vol. 38 (1989) p. 153.

66. D. Lieberman, "Color EL Displays Look Good For '93," Electronic Engineering Times, Issue 621 (Dec 1990) p. 16. 
67. W. A. Barrow, R. E. Coovert, C. N. King and M. J. Ziuchkovski, "Matrix-Addressed Full-Color TFEL Display," Society of Information Display: Digest of Technical Papers, Vol. 19, (1988) p. 284.

68. S. Tanaka, Y. Mikami, H. Deguchi, and H. Kobayashi, "White Light Emitting Thin-Film Electroluminescent Devices with SrS:Ce, Cl/ZnS:Mn Double Phosphor Layers," Japanese J. of Applied Physics, Vol. 25, No. 3 (1986) p. L225.

69. S. Tanaka, Y. Mikami, J. Nishiura, S. Ohshio, H. Yoshiyama, and H. Kobayashi, "A Full-Color Thin-Film EL Device with Two Stacked Substrates and Color Filters," Proceedings of the Society of Information Displays, Vol. 28 , No. 4 (1987) p. 357.

70. S. Tanaka, H. Kawakami, K. Nakamura and H. Kabayashi, "Stable White SrS:Ce, K, Eu TFEL with Filters for Full-Color Devices," Seciety of Information Display: Digest of Technical Papers, Vol. 20, (1989) p. 321.

71. A. Vecht, "A Review of Electroluminescent Thin-Film Frabrication Techniques," Society of Information Display: Digest of Technical Papers, Vol. 20, (1989) p. 304.

72. R. T. Tuenge, "Thin Film Electroluminescent Phosphors for Patterned Full-Color Displays," Springer Proceedings in Physics: Electroluminescence, Vol. 38 (1989) p. 132.

73. T. Suntola, J. Antson, A. Pakkala and S. Lindfors, "Atomic Layer for Producing EL-Thin Films," Seciety of Information Display: Digest of Technical Papers, Vol. 11, (1980) p. 108. 
74. L. F. Zharovsky, L. V. Zavyalova and G. S. Scechnikov, "Metal Chalocogenide Films Prepared from Chelate Organometallic Compounds," Ihin Solid Films, Vol. 128, Nos. 3/4 (1985) p. 241.

75. A. Saunders and A. Vecht, "The Role of Chemical Vapour Deposition in the Fabrication of High Field Electroluminescent Displays," Springer Proceedings in Physics: Electroluminescence, Vol. 38 (1989) p. 210.

76. H. M. Manasevit and W. I. Simpson, "The use of Metal-Organic in the Preparation of Semiconductor Materials," $\downarrow$. of Electrochemical Society, Vol. 118, No. 4 (1971) p. 645.

77, P. J. Wright and B. Cockayne, "The Organometallic Chemical Vapor Deposition of ZnS and ZnSe at Atmospheric Pressure," J. of Crystal Growth, Vol. 59, No. 1/2 (1982) p. 148.

78. A. Yoshikawa, A. Siriai, S. Yamaga and H. Kasai, "Growth Mechanism of ZnS and ZnSe Films in Low-Pressure MOCVD," Japanese J. of Applied Physics, Vol. 25, No. 5 (1986) p. 673.

79. T. Minami, T. Miyata, K. Kitamura, H. Namto and S. Takata, "Low Voltage Driven MOCVD-Grown ZnS:Mn Thin-Film Electroluminescent Devices Using Insulating $\mathrm{BaTiO}_{3}$ Ceramic Sheets," Japanese J. of Applied Physics, Vol. 27, No. 5 (1988) p. L876.

80. P. J. Wright, B. Cockayne, A. F. Cattell, P. J. Dean and A. D. Pitt, "Manganese Doping of ZnS and ZnSe Epitaxial Layers Grown by Organometallic Chemical Vapor Deposition," J. of Crystal Growth, Vol. 59, Nos. 1/2 (1982) p. 155. 
81. A. Yoshikawa, S. Yamaga and K. Tanaka, "New and Simple MOCVD Technique Using Completely Gaseous MO-Source Especially Useful for Growing Zn-Chalcogenide Films," Japanese J. of Applied Physics, Vol. 23, No. 6 (1984) p. L388.

82. M. Migita, O. Kanehisa, M. Shiiki and H. Yamamoto, "The Preparation of $\mathrm{ZnS}: \mathrm{Mn}$ Electroluminescent Layers by MOCVD Using New Manganese Sources," J. of Crystal Growth, Vol. 93 (1988) p. 686.

83. K. Hirabayashi and H. Kozawaguchi, "Photo-Assisted Metal Organic Chemical Vapor Deposition Preparation of Polycrystalline ZnS:Mn Films for Thin Film Electroluminescent Devices," Japanese J. of Applied Physics, Vol. 28, No. 5 (1989) p. 814.

84. K. Hirabayashi and $O$. Kogure, "AC-Thin Film ZnS:Mn Electroluminescent Device Prepared by Metal Organic Chemical Vapor Deposition," Japanese J. of Applied Physics, Vol. 24, No. 11 (1985) p. 1484.

85. R. H. Mauch, R. Menner and H. W. Shock, "Comparison of ZnS:Mn AC TFEL Devices Prepared by Manganese Diffusion and Coevaporation," Lلـ of Crystal Growth, Vol. 86, Nos. $1-4$ (1988) p. 885.

86. K. Hirabayashi and H. Kozawaguchi, "ZnS:Mn Electroluminescent Device Prepared by Metal-Organic Chemical Vapor Deposition," Japanese J. of Applied Physics, Vol. 25, No. 5 (1986) p. 711.

87. W. E. Howard, "The Importance of Insulator Properties in a ThinFilm Electroluminescent Device," IEEE Transactions on Electron Devices, Vol. ED-24, No. 7 (1977) p. 903. 
88. S. K. Tiku, S. H. Rustomji, "Dielectrics for Bright EL Displays," IEEE Transactions on Electron Devices, Vol. ED-36, No. 9 (1989) p. 1947.

89. R. A. Boudreau, J. E. Connolly, B. Dale, "Techniques for Dielectric Strength Monitoring in Electroluminescent Display Manufacture," Society of Information Display Inter. Sym.: Digest of Technical Papers, Vol. 19, (1988) p. 12.

90. M. Yoshida, T. Yamashita, K. Taniguchi, K. Tanaka, T. Ogura, A. Mikami, H. Nakaya, S. Yamaue, and S. Nakajima, "ZnS:Mn Electroluminescent Devices with High Performance Using $\mathrm{SiO}_{2} / \mathrm{Ta}_{2} \mathrm{O}_{5} / \mathrm{SiO}_{2}$ Insulating Layer," Springer Proceedings in Physics: Electroluminescence, Vol. 38 (1989) p. 273.

91. J. Ohwaki, N. Yamauchi, H. Kozawaguchi and B. Tsujiyama, "The Role of Stacked Insulating Layers on Thin-Film Electroluminescent Devices," Japanese J. of Applied Physics, Vol. 26, No. 7 (1987) p. 1064.

92. K. L. Chopra, S. Major and S. K. Pandya, "Review Paper: Transparent Conductors-A Status Review," Thin Solid Films, Vol. 102, No. 1 (1983) p. 1.

93. T. Raycheva and $M$. Nanova, "Etching of $\ln _{2} \mathrm{O}_{3}:$ Sn and $\ln _{2} \mathrm{O}_{3}:$ Te Thin Films in Dilute $\mathrm{HCl}$ and $\mathrm{H}_{3} \mathrm{PO}_{4}$," Thin Solid Films, Vol. 141, No. 2 (1986) p. L87.

94. M. Inoue, T. Matsuoka, Y. Fujita and A. Abe, "Patterning Characteristics of ITO Thin Films," Japanese J. of Applied Physics, Vol. 28, No. 2 (1989) p. 274. 
95. R. Tueta and M. Braguier, "Fabrication and Characterization of Indium Tin Oxide Thin Films for Electroluminescent Applications," Thin Solid Films, Vol. 80, No. 1/2/3 (1981) p. 143.

96. H. U. Habermeier, "Properties of Indium Tin Oxide Thin Films Prepared by Reactive Evaporation," Thin Solid Films, Vol. 80, No. 1/2/3 (1981) p. 157.

97. R. V. Stroh and B. Dolinar, "Lighting the Way," BYTE, Vol. 13, No. 9 (1988) p. 275.

98. Marc Jensen, Materials Engineering Senior Project. San Jose State, 1986.

99. Hoa Do, Materials Engineering Senior Project. San Jose State, 1988.

100. H. Kashani, "Metal-Organic Chemical Vapor Deposition (MOCVD) of Zinc Sulfide Thin Film by Utilizing Dimethylzinc and Hydrogen Sulfide Gases," San Jose State University. Materials Engineering Masters Thesis, December 1988.

101. L. I. Maissel and R. Glang, Handbook of Thin Film Technology, McgrawHill Inc, New York, (1970) p. 1-33.

102. E. Bringuier and A. Geoffroy, "Observation of Double Light Pulses in Thin-Film ZnS:Mn Electroluminescent Devices," Applied Physics Letters, Vol. 48, No. 26 (1986) p. 1780.

103. K. W. Yang and S. J. T. Owen, "Deep Traps and Mechanism of Device Aging in ACTFEL Devices," Proceedings of SID, Vol. 25, No. 1 (1984) p. 7.

104. R. Menn, R. J. Tueta, A. Izrael and M. Braguier, "Thin-Film Electroluminescent Devices: Influence of Mn-Doping Method and 
Degradation Phenomena," Proceedings of SID, Vol. 24, No. 2 (1983) p. 120.

105. G. O. Muller, R. Mach, R. Reetz and G. U. Reinsperger, "Degradation Mechanisms of ac Thin-Film Electroluminescence Displays," Seciety of Information Display International Symposium: Digest of Technical Papers, Vol. 19, (1988) p. 23.

106. Lawrence E. Tannas, Jr., "Failure Modes in ACTFEL Panels," Society of Information Display. International Symposium: Digest of Technical Papers, Vol. 14, (1983) p. 120.

107. J. I. Watanabe, M. Wakitani, S. Sato, and S. Miura, "Analysis of Deterioration in Brightness-Voltage Characteristics of TFEL Displays," Society of Information Display International Symposium: Digest of Technical Papers, Vol. 18, (1987) p. 288.

108. T. Matsuoka, J. Kuwata, M. Nishikawa, Y. Fujita, T. Tohda and A. Abe, "Influence of Oxygen and Metal Oxide Impurities in ZnS:Mn Film on Characteristics of Electroluminescent Devices," Japanese J. of Applied Physics, Vol. 27, No. 8 (1988), p. 1426. 


\section{Appendixes}

\section{A. Thickness Distribution of Prepared ZnS Film Using MOCVD}

Because the introduction of DMZ into the MOCVD reactor is through a nozzle as shown in Fig. 10, the thickness uniformity of prepared $\mathrm{ZnS}$ film is very poor and is distributed radiatively out of a deposition center where the thickness of $\mathrm{ZnS}$ is thickest.

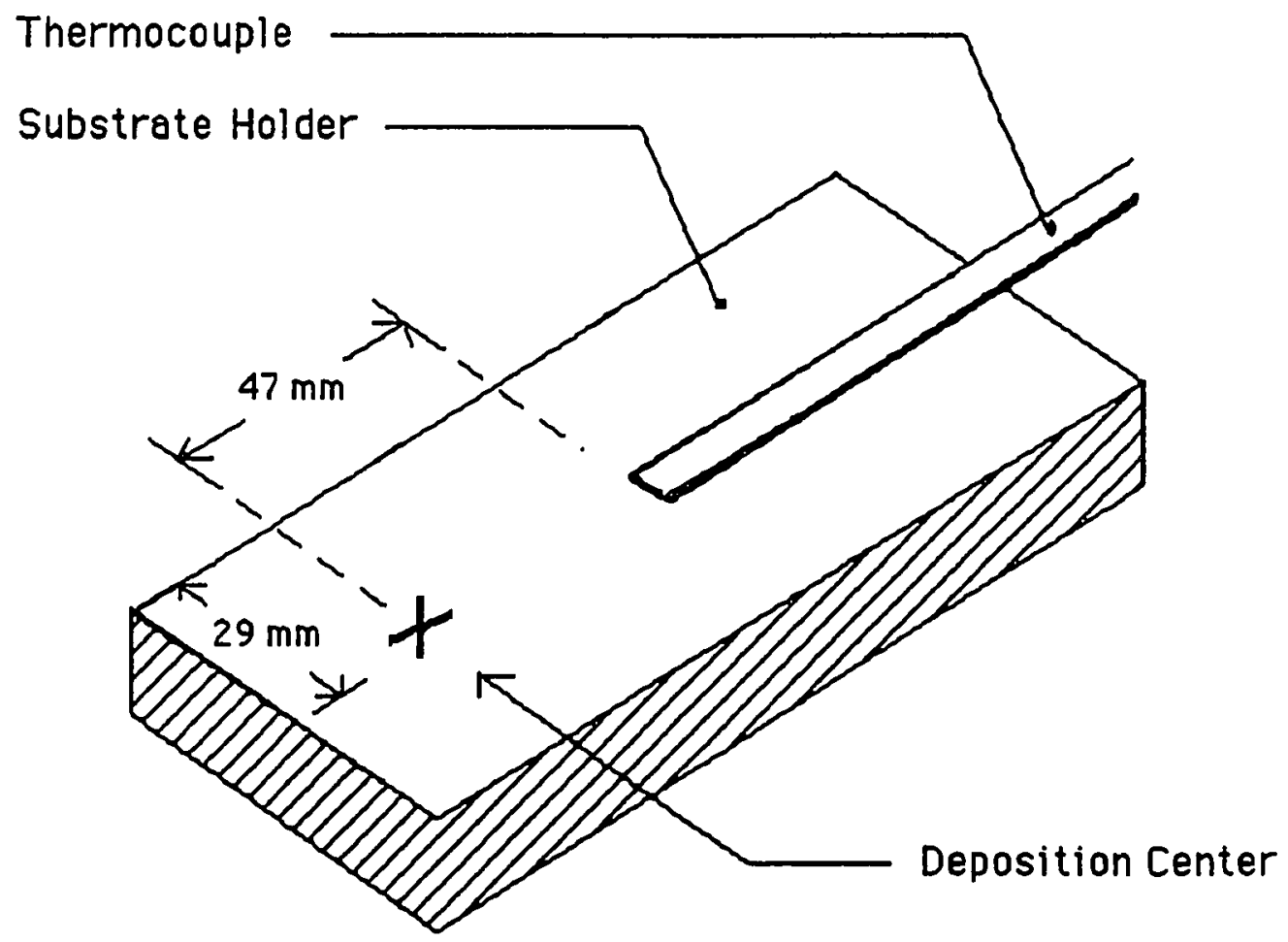

Fig. 18. The relative position of deposition center of MOCVD ZnS. 


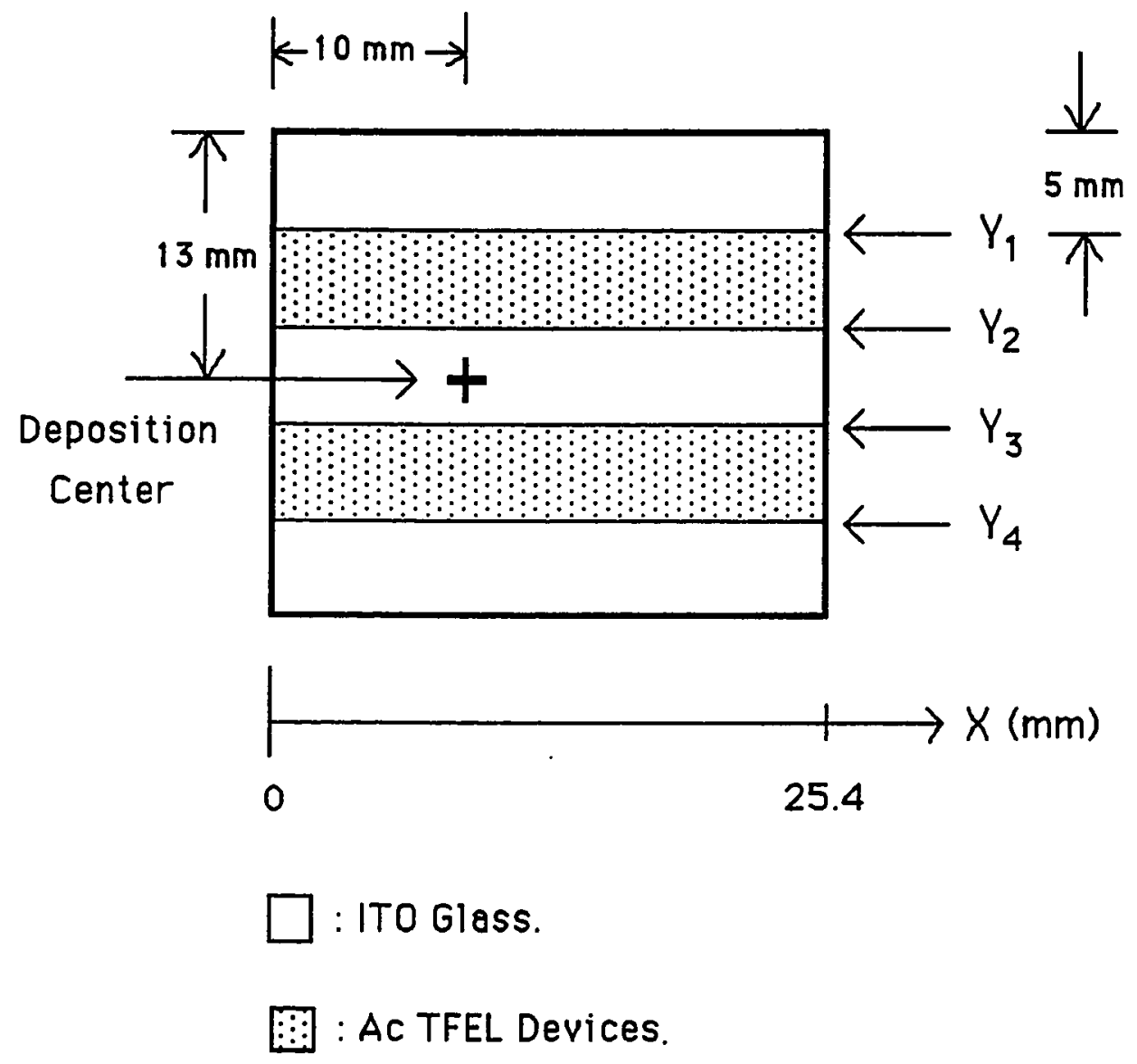

Fig. 19. The schematic illustration of the position of deposition center on ITO glass and the relative positions where measurements of device thickness are made.

The relative position of this center on the substrate holder is shown in Fig. 18 and that on the ITO-glass substrate is shown in Fig. 19. A masked deposition of $\mathrm{ZnS}$ was performed in the MOCVD reactor at temperature $=300{ }^{\circ} \mathrm{C}$, pressure $=1$ torr and time $=18$ minutes, 
after which the thickness measurements were performed at 45 locations on the one-inch-square substrate. The results are summarized in Table 5, and are briefly plotted in Fig. 20. These thickness measurements were performed using an Alpha-step profiler with a measurement error of about plus or minus $5 \times 10^{-6}$ $\mathrm{cm}$.

Table 5. The thickness distribution of MOCVD ZnS.

\begin{tabular}{ccccc}
$\begin{array}{c}\text { Position } \\
\text { in X Axis* } \\
(\mathrm{mm})\end{array}$ & $\mathrm{Y}^{*}{ }^{*}$ & $\mathrm{Y} 2$ & $\mathrm{Y3}$ & $\mathrm{Y} 4$ \\
\hline 4.5 & & (Thickness in $\left.10^{-5} \mathrm{~cm}\right)$ & \\
7 & 13 & 6.5 & 5.5 & 8 \\
9.5 & 18 & 12 & 11 & 13.5 \\
12 & 21 & 18 & 19 & 12.5 \\
14.5 & 16 & 27 & 22 & 11 \\
17 & 14 & 18 & 19.5 & 9.5 \\
19.5 & 9 & 8 & 8 & 5 \\
22 & 2 & 5 & 3.5 & 0.5 \\
24.5 & 1.5 & 3 & 1.5 & - \\
\hline
\end{tabular}

* The measurement positions are indicated in Fig. 19. 


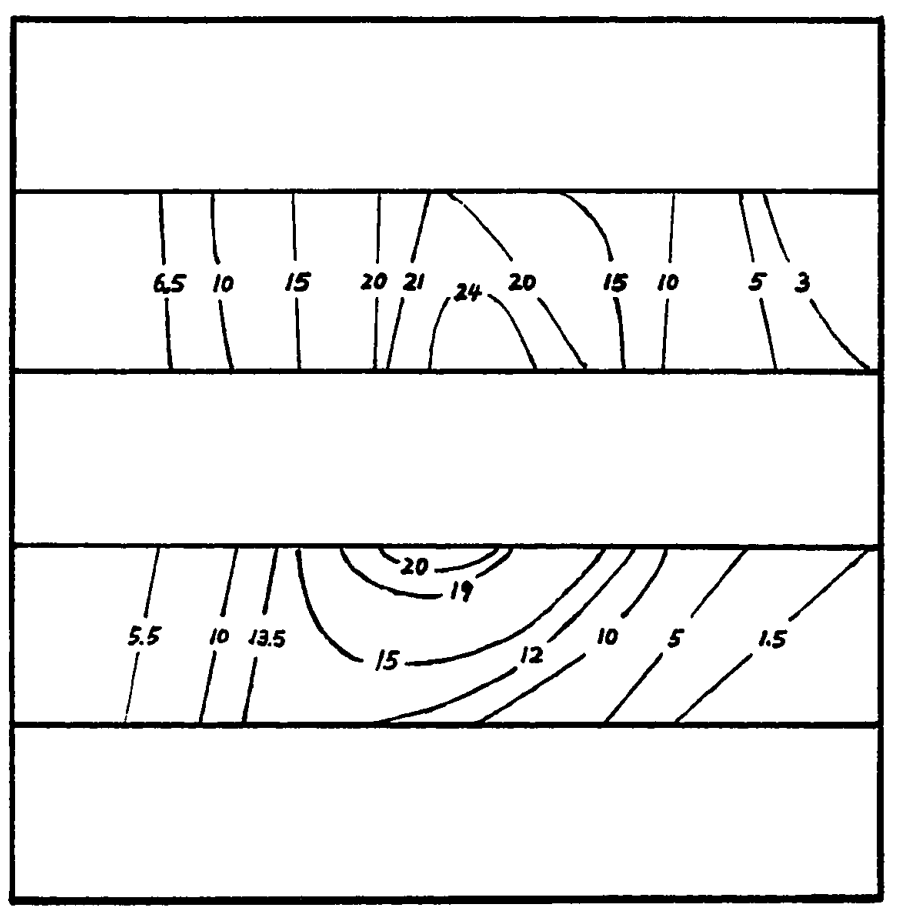

Thickness value in $10^{-5} \mathrm{~cm}$.

Fig. 20. The thickness distribution of MOCVD ZnS. 


\section{B. The Estimation of Evaporated Mn Content in ZnS:Mn}

Because the amount of $\mathrm{Mn}$ to be evaporated is less than $0.01 \mathrm{~g}$ and the evaporation of $\mathrm{Mn}$ onto the surface of $\mathrm{ZnS}$ is carried out at a height equal to $18 \mathrm{~cm}$, the amount of $\mathrm{Mn}$ deposited onto the $\mathrm{ZnS}$ surface can be estimated using the small-area-source approach

$$
d M_{r} / d A_{r}=M_{e} \times \cos (a) \times \cos (b) /\left(\pi \times r^{2}\right)
$$

where $M_{r}$ and $M_{e}$ are the mass of deposit, $M n$, on $Z n S$ at radius $r$ and the total mass of $M n$ to be evaporated, respectively. $A_{r}$ is the area of deposit. $a$ and $b$ are the angles as shown in Fig. 21.(99) If the deposit surface is mounted on a spherical surface of radius $r_{0}$ and the evaporation source is at the center of the sphere, we have $\cos (a)=$ $\cos (b)=r / r_{0}$, and

$$
d M_{r} / d A_{r}=M_{e} /\left(4 \times \pi \times r_{0}^{2}\right)
$$

If $a=b=0^{\circ}$, evaporation height, $h$, will equal $2 r_{0}$ and Eq. A2 can be rewritten as

$$
d M_{r} / d A_{r}=M_{e} /\left(\pi \times h^{2}\right)
$$




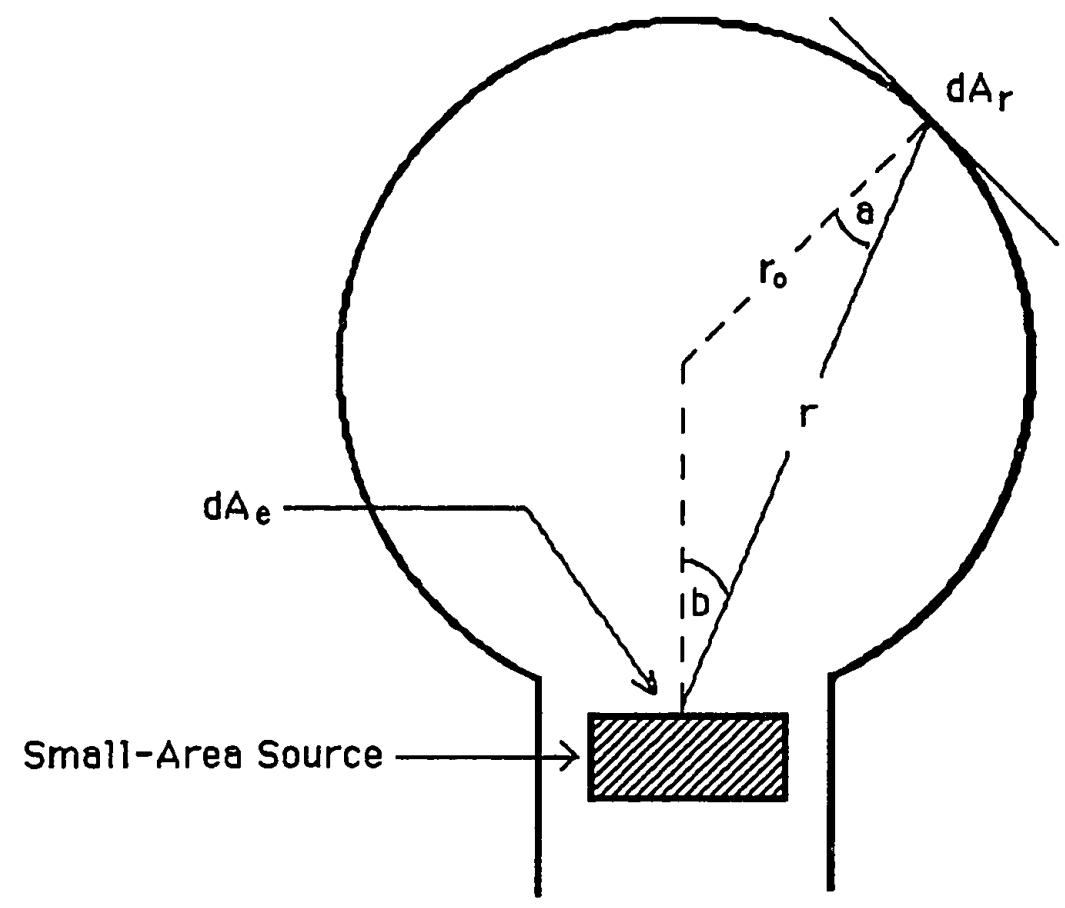

Fig. 21. The schematic illustration of small-area-source approach.

or Eq. A3 can be written as

$$
\begin{aligned}
& \text { Amount of } \mathrm{Mn} \text { deposited on } \mathrm{ZnS} \text { surface }=\Delta \mathrm{Mr}_{\mathrm{r}} \\
& =A_{r} \times M_{e} /\left(\pi \times h^{2}\right)
\end{aligned}
$$

If the deposited $M n$ content is $y \%$ of total amount of $\mathrm{ZnS}+\mathrm{Mn}, \Delta \mathrm{Mr}$ can also be calculated as

$$
\Delta M_{r}=(y \% / 100) \times\left(\text { Amount of } Z n S \text { layer }+\Delta M_{r}\right)
$$


Because the amount of $\mathrm{ZnS}, \mathrm{M}_{\mathrm{ZnS}}$, is much larger than that of $\Delta \mathrm{Mr}_{\mathrm{r}}$, Eq. A5 can be simplified into

$$
\Delta M_{r}=(y \% / 100) \times\left(M_{Z n S}\right)
$$

If the thickness and density of $Z n S$ are $d_{Z n S}$ and $\partial_{Z n s}$, respectively, and the area upon which $\mathrm{Mn}$ is deposited is equal to that of $\mathrm{ZnS}$ layer, $M_{Z n s}$ can be described as

$$
M_{Z n S}=d_{Z n s} \times \partial_{Z n s} \times A_{r}
$$

Combining Eq. A4, $A 6$ and $A 7$, the amount of $M n$ to be evaporated to get $y \% \mathrm{Mn}$ content in $\mathrm{ZnS}$ is given by

$$
M_{e}=(y \% / 100) \times\left(\pi \times h^{2}\right) \times\left(d_{z n s} \times \partial_{z n s}\right)
$$

Because $\partial_{\mathrm{zns}}=3.92 \mathrm{~g} / \mathrm{cm}^{3}$ and $\mathrm{h}=18 \mathrm{~cm}$, Eq. A8 can be expressed as

$$
M_{e}=3.99 \times 10^{-5} \times(y \% / 100) \times\left(d_{Z n s}\right)
$$

where $d_{Z n S}$ is used in $10^{-8} \mathrm{~cm}$ and the unit of $M_{e}$ is in gram. For example, if the thickness of $\mathrm{ZnS}$ is $0.5 \mu \mathrm{m}$, in order to dope 1 weight $\%$ of $\mathrm{Mn}$ into $\mathrm{ZnS}$, the amount of $\mathrm{Mn}$ needed to be evaporated is equal to 0.002 gram. 


\section{The Analysis of Mn Content in ZnS:Mn}

A TFEL device with $\mathrm{ZnS}$ thickness $=3200 \mathrm{~A}$ and $\mathrm{Mn}$ content $=$ 0.0023 gram was analyzed using an Electron Microprobe (by JEOL, model JXA 8600) in IBM by Dennis Ramos. The results are listed in Table 6. The amount of $\mathrm{Mn}$ in $\mathrm{ZnS}: \mathrm{Mn}$ was found to be 2.0 wt. \%.

Table 6. Analysis of $\mathrm{Mn}$ content in $\mathrm{ZnS:Mn}$ using Electron Microprobe.

\begin{tabular}{cccc}
$\begin{array}{c}\text { Data } \\
\text { Point }\end{array}$ & $\begin{array}{c}\mathrm{Zn} \\
(\text { wt. \%) }\end{array}$ & $\begin{array}{c}\mathrm{S} \\
(\text { wt. \%) }\end{array}$ & $\begin{array}{c}\mathrm{Mn} \\
(\text { wt. \%) }\end{array}$ \\
\hline 1 & 66.60 & 31.40 & 2.00 \\
2 & 66.57 & 31.46 & 1.97 \\
3 & 67.52 & 30.50 & 1.98 \\
4 & 66.83 & 31.16 & 2.01 \\
5 & 66.85 & 31.21 & 1.94 \\
6 & 66.65 & 31.36 & 1.99 \\
7 & 66.96 & 31.06 & 1.98 \\
8 & 67.04 & 30.95 & 2.01 \\
9 & 67.24 & 30.72 & 2.04 \\
10 & 67.06 & 30.91 & 2.03 \\
Average & 66.93 & 31.07 & 2.00 \\
\hline
\end{tabular}


The $\mathrm{Mn}$ content in $\mathrm{ZnS}$ throughout this work was estimated using Eq. (A9),

$$
M_{e}=3.99 \times 10^{-5} \times(y \% / 100) \times\left(d_{z n s}\right)
$$

or

$$
y \%=2.506 \times 10^{6} \times M_{e} / d_{Z n s}
$$

From this equation, the $M n$ content of a TFEL device with $d_{Z n s}=3200$ $A$ and $M_{e}=0.0023 \mathrm{~g}$ can be calculated to be $1.84 \mathrm{wt}$. \%. This amount is closed to the value measured using Electron Microprobe--2.0 wt. \%. Therefore, Eq. (A9) is valid for estimating Mn content.

Other information given in Table 6 is the stoichiometry of MOCVD ZnS. If we convert the amount of $\mathrm{Zn}$ and $\mathrm{S}$ in Table 6 from weight percent to atomic percent, the atomic ratio of $\mathrm{Zn}$ to $S$ is 1 : 0.95 . This result shows that the stoichiometry of MOCVD prepared ZnS using the proposed operation conditons is good. 


\section{The Oxygen Content in ZnS:Mn}

Because of the limitation of the facility, the introduction of impurities other than $\mathrm{Mn}$ in $\mathrm{ZnS}$ layers was unavoidable during the device fabrication. Another analysis using Electron Microprobe shows the existence of oxygen. The results are listed in Table 7, which shows that the oxygen content in $\mathrm{ZnS}: \mathrm{Mn}$ is around $18.5 \mathrm{wt}$ \%. This existence of oxygen in $\mathrm{ZnS}: \mathrm{Mn}$ is believed to form an additional insulating layer such as $\mathrm{ZnSO}_{4}{ }^{(108)}$.

Table 7. Analysis of oxygen content in $\mathrm{ZnS}: \mathrm{Mn}$.

\begin{tabular}{ccccc}
$\begin{array}{c}\text { Data } \\
\text { Point }\end{array}$ & $\begin{array}{c}\mathrm{Zn} \\
\text { (atomic \%) }\end{array}$ & $\begin{array}{c}\text { S } \\
\text { (at. \%) }\end{array}$ & $\begin{array}{c}\text { (at. \%) } \\
\text { (at. \%) }\end{array}$ \\
\hline 1 & 40.88 & 38.98 & 1.4 & 18.65 \\
2 & 41.12 & 38.97 & 1.44 & 18.47 \\
Average & 41.0 & 39.0 & 1.5 & 18.5 \\
\hline
\end{tabular}

